\title{
PHISICS/RELAP5-3D Adaptive Time-Step Method Demonstrated for the HTTR LOFC\#1 Simulation
}

R. I. Baker and P. Balestra

The INL is a

U.S. Department of Energy

National Laboratory

operated by

Battelle Energy Alliance

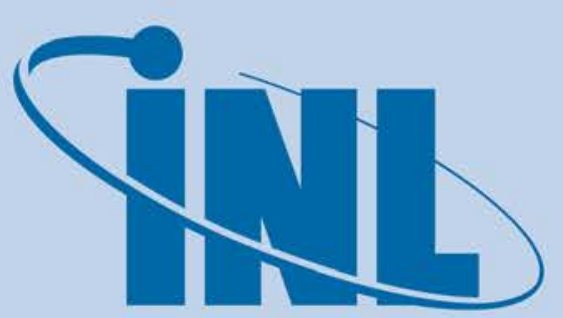

Idaho National Laboratory

May 2017

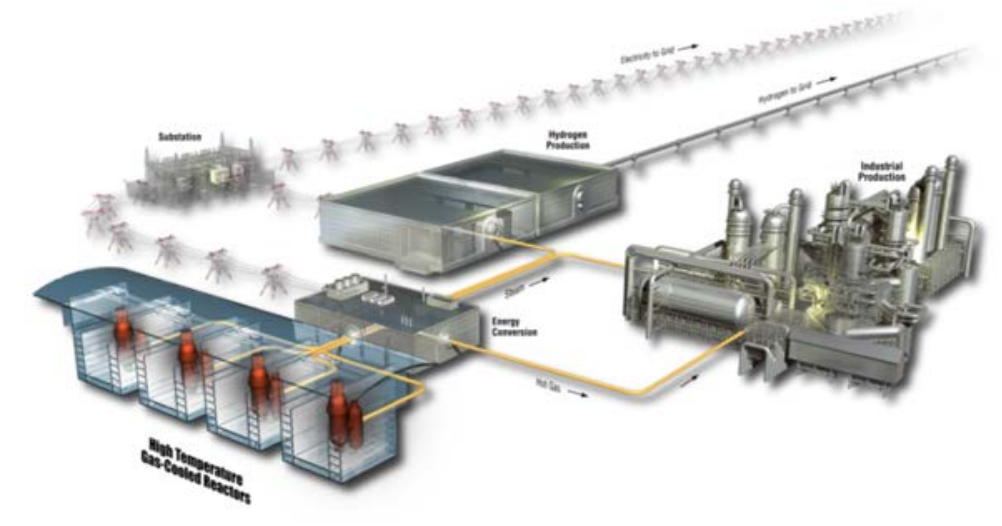




\section{DISCLAIMER}

This information was prepared as an account of work sponsored by an agency of the U.S. Government. Neither the U.S. Government nor any agency thereof, nor any of their employees, makes any warranty, expressed or implied, or assumes any legal liability or responsibility for the accuracy, completeness, or usefulness, of any information, apparatus, product, or process disclosed, or represents that its use would not infringe privately owned rights. References herein to any specific commercial product, process, or service by trade name, trade mark, manufacturer, or otherwise, does not necessarily constitute or imply its endorsement, recommendation, or favoring by the U.S. Government or any agency thereof. The views and opinions of authors expressed herein do not necessarily state or reflect those of the U.S. Government or any agency thereof. 
INL/EXT-17-41569

Revision 0

\title{
PHISICS/RELAP5-3D Adaptive Time-Step Method Demonstrated for the HTTR LOFC\#1 Simulation
}

\author{
R. I. Baker and P. Balestra
}

May 2017

Idaho National Laboratory

INL ART TDO Program

Idaho Falls, Idaho 83415

http://www.inl.gov

Prepared for the

U.S. Department of Energy

Office of Nuclear Energy

Under DOE Idaho Operations Office

Contract DE-AC07-05ID14517 



\title{
INL ART TDO Program \\ PHISICS/RELAP5-3D \\ Adaptive Time-Step Method Demonstrated for the HTTR LOFC\#1 Simulation
}

\author{
INL/EXT-17-41569 \\ Revision 0
}

May 2017

Authors:

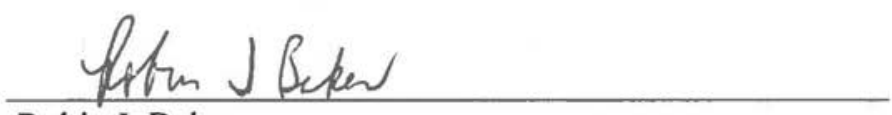

Robin I. Baker

INL ART TDO Graduate Intern, Nuclear Engineering

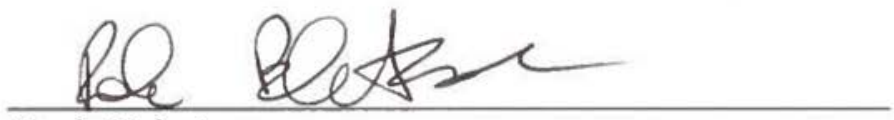

Paolo Balestra

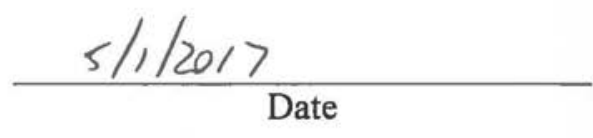

University of Rome

\section{$5 / 1 / 2017$ \\ Date}

Technical Rêtiewer:

Gerhard Strydom

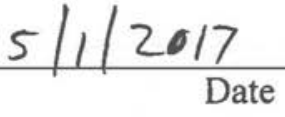

INL ART YDO Design Methods Lead

Approved By:
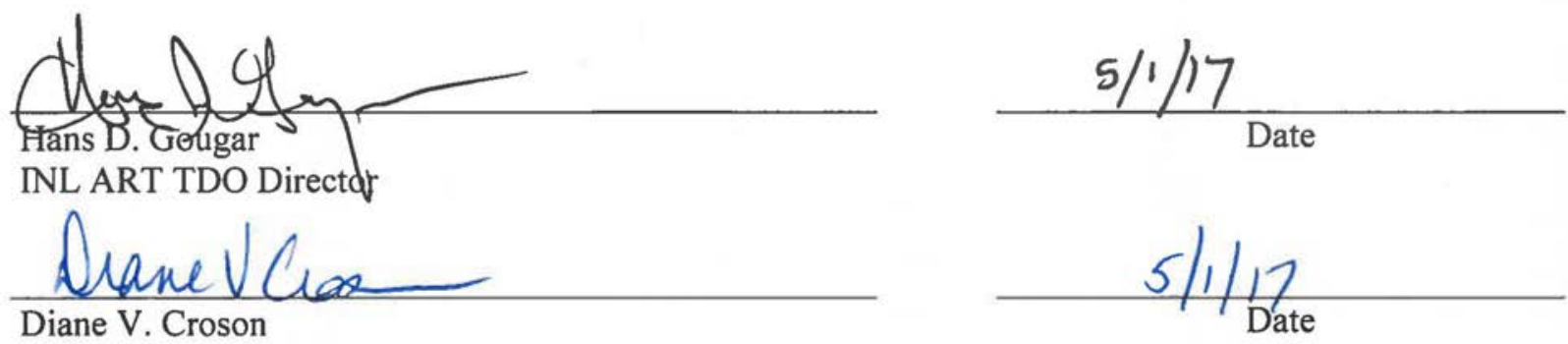

Diane V. Croson

INL ART TDO Deputy Director
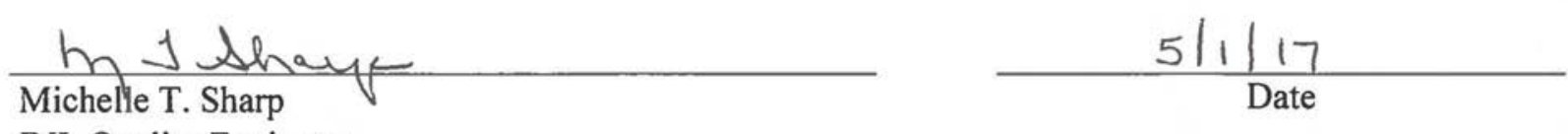

INL Quality Engineer 



\begin{abstract}
A collaborative effort between Japan Atomic Energy Agency and Idaho National Laboratory, as part of the Civil Nuclear Energy Working Group, is underway to model the high temperature engineering test reactor (HTTR) loss of forced cooling (LOFC) transient that was performed in December 2010. The coupled version of RELAP5-3D (i.e., a thermal fluids system code) and PHISICS (i.e., a three-dimensional neutron kinetics code) were used to simulate the transient.

The focus of this report is to summarize the changes made to the PHISICSRELAP5-3D code for implementing an adaptive time-step method into the code for the first time and to test it using the full HTTR PHISICS/RELAP5-3D model developed by Japan Atomic Energy Agency and Idaho National Laboratory and the LOFC simulation. Various adaptive schemes are available based on flux or power convergence criteria that allow significantly larger time steps to be taken by the neutronics module. This report includes a description of the HTTR and the associated PHISICS/RELAP5-3D model test results, as well as the University of Rome report documenting the adaptive time-step theory and method implemented in PHISICS/RELAP5-3D.

Two versions of the HTTR model were tested using 8- and 26-energy groups. Most of the new adaptive methods lead to significant improvements in the LOFC simulation time required, without significant accuracy penalties in the prediction of fission power and fuel temperature. In the best performing 8-group model scenarios, an LOFC simulation of 20 hours could be completed in real-time, or even less than real-time, compared with the previous version of the code that completed the same transient three to eight times slower than real-time.

However, a few of the method and tolerance combinations resulted in unacceptably high errors or insignificant gains in simulation time. The study is concluded with recommendations on which methods to use for this HTTR model. An important caveat is that these findings are very model-specific and cannot be generalized to other PHISICS/RELAP5-3D models.
\end{abstract}




\section{ACKNOWLEDGEMENTS}

The authors would like to acknowledge the assistance of Dr. Andrea Alfonsi (main PHISICS/RELAP5-3D code developer) with continual improvement of the code. The HTTR RELAP5-3D model was developed with the guidance of Paul Bayless. 


\section{CONTENTS}

ABSTRACT vii

ACKNOWLEDGEMENTS ix

ACRONYMS xvii

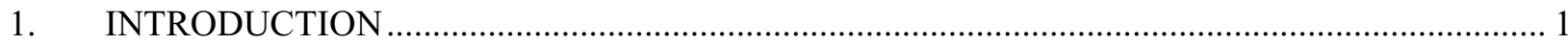

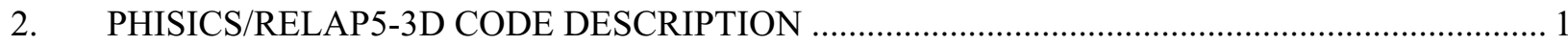

3. OVERVIEW OF ADAPTIVE TIME-STEP METHOD AND TEST CASES .............................. 2

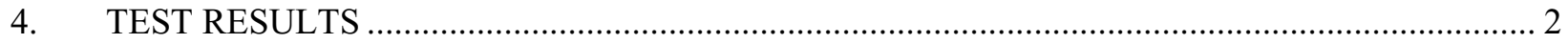

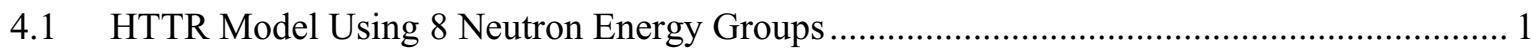

4.1.1 Methods 1, 2, and 3 Using "flux_toll" Option ............................................................ 1

4.1.2 Method 1 Using "flux_toll" Option with Additional Tolerance Values ..................... 6

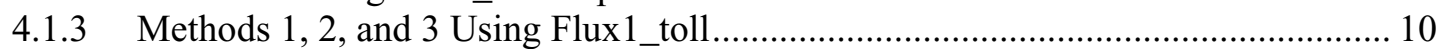

4.1.4 Method 1 Using Flux1_toll and Varying the Tolerance ............................................ 15

4.1.5 Method 2 Using Maximum Time Step Between Flux_toll and Flux1_toll................ 18

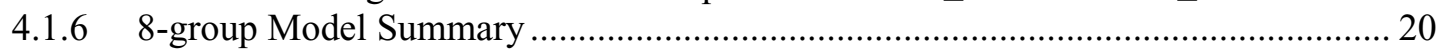

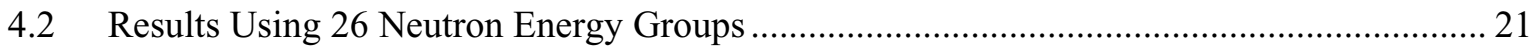

4.2.1 Methods 1, 2, and 3 Using Flux_toll and a Tolerance of 1E-5 ............................. 21

4.2.2 Method 1 Using Flux_toll and Varying the Tolerance .......................................... 23

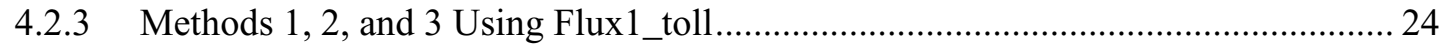

4.2.4 Method 1 Using Flux1_toll and Varying the Tolerance ......................................... 27

4.2.5 Method 2 Using Maximum Time Step Between Flux_toll and Flux1_toll............... 29

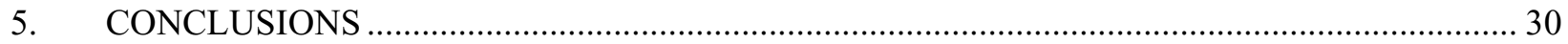

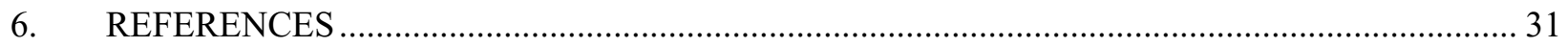

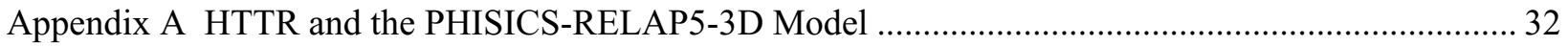

Appendix B Implementation of an Adaptive Time Step Methodology for the PHISICS/RELAP5-3D Coupling Scheme.

\section{FIGURES}

Figure 1. Fission power (MW) for the reference case and Methods 1, 2, and 3 using flux_toll with a tolerance of $1 \mathrm{E}-5$

Figure 2. Ring 1 fuel block average temperature $(\mathrm{K})$ at the approximate axial mid-point for the reference case and Methods 1, 2, and 3 using flux_toll with a tolerance of 1E-5.

Figure 3. Fission power difference $(\mathrm{kW})$ between the reference case and Methods 1, 2, and 3 using flux_toll with a tolerance of $1 \mathrm{E}-5$. 3 
Figure 4. Fission power percent difference normalized to the steady state fission power between the reference case and Methods 1, 2, and 3 using flux_toll with a tolerance of 1E-5.

Figure 5. Fission power percent difference in the first 1.8 hours between the reference case and Methods 1, 2, and 3 using flux_toll with a tolerance of $1 \mathrm{E}-5$

Figure 6. Fission power percent difference in the re-criticality period between the reference case and Methods 1, 2, and 3 using flux_toll with a tolerance of 1E-5.

Figure 7. Fuel temperatures percent difference comparison between the reference to Methods 1, 2, and 3 using flux_toll with a tolerance of 1E-5 5

Figure 8. Time enhancement factor using a ratio of the cpu time to the problem time plotted over time for Methods 1, 2, and 3 using flux_toll with a tolerance of 1E-5......

Figure 9. Fission power (MW) for the reference case and Method 1 flux_toll using three different tolerance values: $1 \mathrm{E}-4,1 \mathrm{E}-5$, and $1 \mathrm{E}-6$.

Figure 10. Ring 1 fuel block average temperature $(\mathrm{K})$ at the approximate axial mid-point for the reference case and Method 1 using flux toll and three different tolerance values: $1 \mathrm{E}-4$, $1 \mathrm{E}-5$, and $1 \mathrm{E}-6$.

Figure 11. Fission power percent difference (from the reference case) for Method 1 using flux_toll with three different tolerance values: $1 \mathrm{E}-4,1 \mathrm{E}-5$, and $1 \mathrm{E}-6$. .

Figure 12. Fuel temperature percent difference (from the reference case) for Method 1 using flux_toll with three different tolerance values: $1 \mathrm{E}-4,1 \mathrm{E}-5$, and $1 \mathrm{E}-6$.

Figure 13. Time enhancement for the reference case and Method 1 using flux_toll and three different tolerance values: $1 \mathrm{E}-4,1 \mathrm{E}-5$, and $1 \mathrm{E}-6$.

Figure 14. Fission power (MW) for the reference case and Methods 1, 2, and 3 using flux1_toll with a tolerance of $1 \mathrm{E}-4$.

Figure 15. Fission power (MW) for the initial transient for the reference case and Methods 1, 2, and 3 using flux 1 toll with a tolerance of $1 \mathrm{E}-4$......

Figure 16. Fission power during the re-criticality portion of the transient for the reference case and Methods 1, 2, and 3 using flux1_toll with a tolerance of 1E-4.

Figure 17. Fission power percent difference (from the reference case) for Methods 1, 2, and 3 using flux1_toll with a tolerance of $1 \mathrm{E}-4$.

Figure 18. Fission power percent difference (from the reference case) in the re-criticality phase of the transient for Methods 1, 2, and 3 using flux1_toll with a tolerance of 1E-4.

Figure 19. Ring 1 fuel block average temperature $(\mathrm{K})$ at the approximate axial mid-point for the reference case and Methods 1, 2, and 3 using flux1_toll with a tolerance of 1E-4.

Figure 20. Fuel temperature percent difference (from the reference case) for Methods 1, 2, and 3 using flux1_toll with a tolerance of 1E-4.

Figure 21. Time enhancement factor using a ratio of the cpu time to the problem time plotted over time for Methods 1, 2, and 3 using flux1_toll with a tolerance of 1E-4.

Figure 22. Fission power for the reference case and Method 1 flux1_toll using three different tolerance values: $1 \mathrm{E}-3,1 \mathrm{E}-4$, and $1 \mathrm{E}-5$.

Figure 23. Fission power during the re-criticality period for the reference case and Method 1 flux1_toll using three different tolerance values: $1 \mathrm{E}-3,1 \mathrm{E}-4$, and $1 \mathrm{E}-5$. 16 
Figure 24. Fission power percent difference (from the reference case) for Method 1 using flux1_toll and three different tolerance values: $1 \mathrm{E}-3,1 \mathrm{E}-4$, and $1 \mathrm{E}-5$.

Figure 25. Ring 1 fuel block average temperature $(\mathrm{K})$ at the approximate axial core mid-point for the reference case and Method 1 flux1_toll using three different tolerance values:

$1 \mathrm{E}-3,1 \mathrm{E}-4$, and $1 \mathrm{E}-5$.

Figure 26. Time enhancement for the reference case and Method 1 using flux1_toll and three different tolerance values: $1 \mathrm{E}-3,1 \mathrm{E}-4$, and $1 \mathrm{E}--5$.

Figure 27. Fission power for the reference case and Method 2 using maximum time step between flux_toll and flux1_toll with tolerances of $1 \mathrm{E}-5$ and $1 \mathrm{E}-4$, respectively.

Figure 28. Fission power percent difference (from the reference case) for Method 2 using the maximum time step between flux_toll and flux1_toll with tolerances of $1 \mathrm{E}-5$ and $1 \mathrm{E}-4$, respectively.

Figure 29. Time enhancement for the reference case and Method 2 using the maximum time step calculation between flux_toll and flux1_toll with tolerances of $1 \mathrm{E}-5$ and $1 \mathrm{E}-4$, respectively.

Figure 30. Time enhancement factor using a ratio of the cpu time to the problem time plotted over time for Methods 1, 2, and 3 using flux_toll with a tolerance of 1E-5.

Figure 31. Fission power (MW) for the reference case and Methods 1, 2, and 3 using flux_toll with a tolerance of $1 \mathrm{E}-5$.

Figure 32. Ring 1 fuel block average temperature $(\mathrm{K})$ at the approximate axial mid-point for the reference case and Methods 1, 2, and 3 using flux_toll with a tolerance of 1E-5.

Figure 33. Fission power (MW) for the reference case and Method 1 flux_toll using three different tolerance values: $1 \mathrm{E}-4,1 \mathrm{E}-5$, and $1 \mathrm{E}-6$.

Figure 34. Time enhancement for the reference case and Method 1 using flux_toll and three different tolerance values: $1 \mathrm{E}-4,1 \mathrm{E}-5$, and $1 \mathrm{E}-6$.

Figure 35. Time enhancement factor using a ratio of the cpu time to the problem time plotted over time for Methods 1, 2, and 3 using flux1_toll with a tolerance of $1 \mathrm{E}-4$.

Figure 36. Fission power (MW) for the reference case and Methods 1, 2, and 3 using flux1_toll with a tolerance of $1 \mathrm{E}-4$.

Figure 37. Fission power during the re-criticality portion of the transient for the reference case and Methods 1, 2, and 3 using flux1_toll with a tolerance of 1E-4. 26

Figure 38. Ring 1 fuel block average temperature $(\mathrm{K})$ at the approximate axial mid-point for the reference case and Methods 1, 2, and 3 using flux1_toll with a tolerance of $1 \mathrm{E}-4$.

Figure 39. Fission power for the reference case and Method 1 flux1_toll using three different tolerance values: $1 \mathrm{E}-3,1 \mathrm{E}-4$, and $1 \mathrm{E}-5$.

Figure 40. Fission power during the re-criticality period for the reference case and Method 1 flux1_toll using three different tolerance values: $1 \mathrm{E}-3,1 \mathrm{E}-4$, and $1 \mathrm{E}-5$. .28

Figure 41. Time enhancement for the reference case and Method 1 using flux1_toll and three different tolerance values: $1 \mathrm{E}-3,1 \mathrm{E}-4$, and $1 \mathrm{E}-5$.

Figure 42. Fission power for the reference case and Method 2 using the maximum time step calculated using flux_toll and flux 1 _toll with tolerances of $1 \mathrm{E}-5$ and $1 \mathrm{E}-4$, respectively. 
Figure 43. Time enhancement for the reference case and Method 2 using the maximum time step calculation between flux_toll and flux1_toll with tolerances of $1 \mathrm{E}-5$ and $1 \mathrm{E}-4$, respectively.

Figure A-1. Cutaway of a HTTR compact and prismatic fuel block..................................................... 34

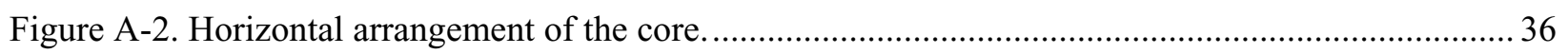

Figure A-3. Core arrangement of the HTTR. The underlined numbers (e.g., 6, 6, 12, and 6 under the different type of fuel elements) denote the total number of blocks. ................................... 36

Figure A-4. Reactor core internals of the High Temperature Engineering Test Reactor. .......................... 37

Figure A-5. Vertical view of High Temperature Engineering Test Reactor. ............................................. 38

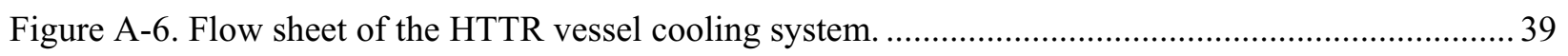

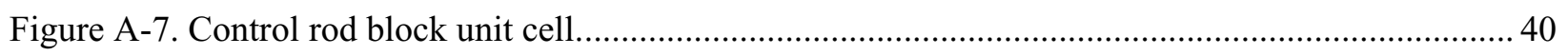

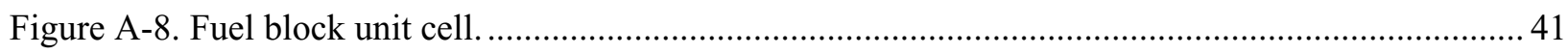

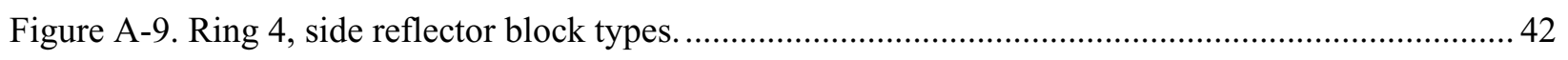

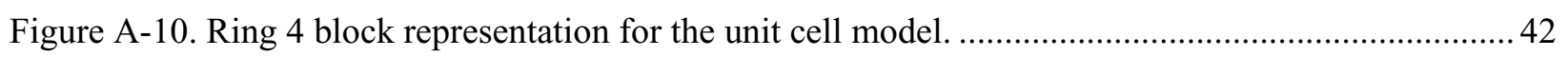

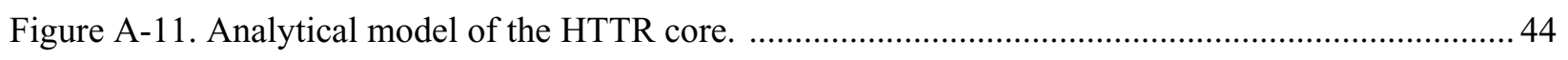

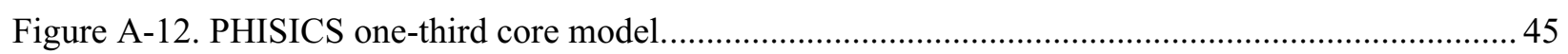

\section{TABLES}

Table 1. 8- and 26-neutron energy group upper energy boundaries. .................................................... 3

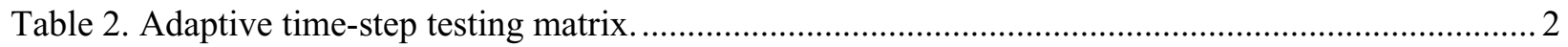

Table 3. Run time summary for Methods 1, 2 and 3 using flux_toll with a tolerance of 1E-5................... 6

Table 4. Run time summary for Method 1 using flux_toll with tolerance values of 1E-4, 1E-5, and

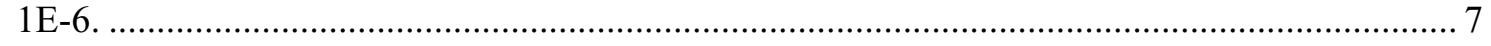

Table 5. Run time summary for Methods 1, 2 and 3 using flux1_toll with a tolerance of $1 \mathrm{E}-4 \ldots \ldots \ldots \ldots \ldots . . . .10$

Table 6. Run time summary for Method 1 using flux 1_toll with tolerances of $1 \mathrm{E}-3,1 \mathrm{E}-4$, and $1 \mathrm{E}-5$.

Table 7. Run time summary for Method 2 using maximum time step between flux_toll (tolerance $=1 \mathrm{E}-5)$ and flux1_toll (tolerance $=1 \mathrm{E}-4)$.

Table 8. Run time summary for Methods 1, 2 and 3 using flux_toll with a tolerance of 1E-5.......

Table 9. Run time summary for Method 1 using flux_toll with tolerance values of 1E-4, 1E-5, and $1 \mathrm{E}-6$.

Table 10. Run time summary for the 26-group simulation using Methods 1, 2 and 3 and flux1_toll with a tolerance of $1 \mathrm{E}-4$.

Table 11. Run time summary for Method 1 using flux1_toll with tolerances of $1 \mathrm{E}-3,1 \mathrm{E}-4$, and $1 \mathrm{E}-5$.

Table 12. Run time summary for Method 2 using maximum time step between flux_toll (tolerance $=1 \mathrm{E}-5)$ and flux1_toll (tolerance $=1 \mathrm{E}-4)$. 
Table A-1. High Temperature Engineering Test Reactor design parameters at $9 \mathrm{MW}$.

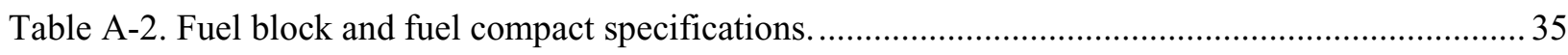




\section{ACRONYMS}

cpu central processing unit

CR control rod

ESF engineered safety features

HPC high performance computing

HTTR High Temperature Engineering Test Reactor

INL Idaho National Laboratory

JAEA Japan Atomic Energy Agency

LOFC loss of forced cooling

MRTAU Multi-Reactor Transmutation Analysis Utility

PHISICS Parallel and Highly Innovative Simulation for INL Code System

RELAP Reactor Excursion and Leak Analysis Program

RPV reactor pressure vessel

RSS reserve shutdown system

VCS vessel cooling systems 
xviii 


\section{PHISICS/RELAP5-3D}

\section{Adaptive Time-Step Method Demonstrated for the HTTR LOFC\#1 Simulation}

\section{INTRODUCTION}

Modeling and simulation of Japan's High Temperature Engineering Test Reactor (HTTR) is being performed at Idaho National Laboratory (INL) as part of a cooperative effort between Japan and the United States under the Civil Nuclear Energy Working Group. The safety analysis code RELAP5-3D (Reactor Excursion and Leak Analysis Program), coupled with INL's reactor physics code PHISICS (Parallel and Highly Innovative Simulation for INL Code System), is being used to model the loss of forced cooling (LOFC) transient experiment completed on December 21, 2010. This experiment is the first of three LOFC planned experiments. A comprehensive report describing the status of the HTTR LOFC\#1 modeling efforts at INL was issued December 2016 (Reference 1) with limited distribution due to the proprietary nature of the LOFC\#1 experiment data. This current report is focused on changes implemented in PHISICS/RELAP5-3D and associated test results using the HTTR model, without reference to experimental data. A description of the HTTR and the PHISICS-RELAP5-3D model is included as Appendix A as background to readers who might not have access to the December 2016 report.

The time required to run the HTTR PHISICS/RELAP5-3D LOFC\#1 model can vary from a few hours to several days, depending on the number of neutron energy groups and the fidelity of the thermal model being used in the calculations. Therefore, a reduction of these very long transient run times would be beneficial for model development, early scoping calculations and validation comparisons. The existing time-step methodology used in PHISICS/RELAP5-3D was first modified so that the thermal fluidic time steps taken by the RELAP5-3D module could be separated, or decoupled, from the neutronics/kinetics time steps taken by the PHISICS solver to support a faster calculation time. Depending on the complexity of the thermal model and the number of energy groups specified by the user, the time spent by these two solvers in the iteration scheme varies significantly. In previous versions of PHISICS/RELAP5-3D, the neutronics solver was limited to the largest time step employed in the RELAP5-3D module, a value bounded by the Courant limit. By decoupling these two solvers, the neutronics module is not required to run as many interim calculations and therefore, reduces the number of relatively expensive source problems to be solved.

In order for the neutronics solver to determine the optimal time step size it could take and still achieve acceptable accuracy levels, various adaptive time-step method were implemented in PHISICS/RELAP53D based on flux and power tolerance criteria. These schemes are specified by the user as part of a new RELAP5-3D input block. The relative performance of these methods is systematically compared in this report to the original (i.e., unmodified) coupled calculation, using the reactor power and fuel temperature in a fixed location as main figures of merit. Note that the adequacy of the current model to simulate the experimental LOFC\#1 transient is not addressed in this report.

Theory development, code implementation and initial testing on a small HTTR-equivalent model were performed by Dr. P. Balestra under a sub-contract with the University of Rome. His report describing the adaptive time-step methodology is included as Appendix B. ${ }^{2}$

\section{PHISICS/RELAP5-3D CODE DESCRIPTION}

RELAP5-3D is a thermal fluidics system code developed at INL for best-estimate transient simulation of light water reactors. The code models reactor systems and core thermal fluidics, along with the neutron kinetics of the reactor, such that a coupled response to a transient may be simulated. Nodal neutron 
kinetics modeling within RELAP5-3D is based on the NESTLE code ${ }^{3}$, which is limited to solving a two or four group neutron diffusion equations in Cartesian or hexagonal geometry. Prior modeling indicates this level of fidelity is not sufficient to obtain an accurate power profile for a high temperature gas cooled reactor ${ }^{4}$.

PHISICS is a time-dependent reactor physics package developed at $\mathrm{INL}^{5}$. It consists of many modules, however, the main three used for this simulation include: (1) a nodal and semi-structured spherical harmonics based transport core solver (INSTANT), (2) a depletion module (Multi-Reactor Transmutation Analysis Utility [MRTAU]), and (3) a cross-section mixer-interpolator module (Mixer). Each module is fully parallelized to run on shared memory computers or medium-to-high performance computing (HPC) platforms. During the development of PHISICS/RELAP5-3D, it was decided to couple the different modules of PHISICS directly to RELAP5-3D (i.e., PHISICS is integrated in RELAP5-3D as a module).

The INSTANT transport solver is parallelized and based on the second order formulation of the transport equation in which the scattering angle is approximated with spherical harmonics and the flux is expanded in orthonormal polynomials of an arbitrary order. A time-dependent scheme was recently implemented as a new module for the PHISICS suite, based on a second order backward Euler scheme with explicit delayed neutron treatment.

The MRTAU module is a generic depletion code developed at INL to perform transmutation, decay and other depletion related calculations. The code tracks the time evolution of the isotopic concentration of a given material and utilizes a Taylor series expansion based algorithm of arbitrary order and the Chebyshev Rational Approximation Method for computation of the exponential matrix. The MIXER module performs all cross-section handling for the different kernels. This module can treat macroscopic, microscopic, and "mixed" cross sections. More detailed descriptions of the PHISICS/RELAP5-3D coupling methodology can be found in Reference 5.

\section{OVERVIEW OF ADAPTIVE TIME-STEP METHOD AND TEST CASES}

The current scheme is based on a first order backward Euler scheme with explicit delayed neutron treatment. Appendix B provides the full report describing the adaptive time-step method based on this scheme. An overview of the time-step method tested using the HTTR model is provided below. Decoupling of thermal fluidic time steps from the neutronics/kinetic solver time step allows a faster calculation time with the acceptable error level determined by a user-defined tolerance value.

Three main adaptive time-step methods were developed based on the truncation error estimation using the second derivative of the flux. The difference between each method is contained in the technique used to estimate the second derivative. Method 1 uses a simple backward scheme, Method 2 uses a combination of backward and forward first order schemes, and Method 3 uses a combination of backward and centered first order schemes.

Within each of the three methods, there are four different tolerance options for calculating the time step. Two of the four options were incorporated into the testing performed: "flux_toll" and "flux1_toll", based on earlier test results performed with a smaller HTTR-based model and recommendation from the developer.

The time step using flux_toll is calculated such that the integrated truncation error on the flux will be smaller than the tolerance " $\epsilon$ " specified by the user.

$\Delta t^{(n+2)}=\sqrt{2 \epsilon \phi^{(n+1)}\left|\frac{\partial^{2} \phi^{(n+1)}}{\partial t^{2}}\right|^{-1}}$ 
Four different sub-tolerance types are allowed; however, only the "loc" option to calculate the time step in each subdomain/energy group, with the smallest one selected to maintain the error below the tolerance in each subdomain/energy group, was tested. The "loc" option is the most conservative and the only one that maintains the error within the tolerance for each node.

The flux1_toll option calculates the time step so the truncation error on the flux is smaller than the tolerance " $\tau$ ".

$$
\Delta t^{(n+2)}=2 \tau\left|\frac{\partial^{2} \phi^{(n+1)}}{\partial t^{2}}\right|^{-1}
$$

The tolerance is normalized with the average flux of the first iteration; therefore, the tolerance must be specified as a relative value (e.g., 1E-1, 1E-3, and 1E-4) of the initial average flux. The same four subtolerance types as flux_toll are allowed, and only the "loc" option was tested.

Further, there is an option for selecting the minimum or maximum time step as calculated by two tolerance options used for the transient calculation. This option was included as part of the testing using Method 2 based on results obtained by the developer on the smaller scale HTTR model.

Each of the three methods was tested using the "full" HTTR LOFC\#1 PHISICS/RELAP5-3D model (compared to a scaled version referenced in the Appendix B report describing the adaptive time-step method changes). The HTTR LOFC\#1 model is described in Appendix A. Two variants of the neutronics section of the model were created, using 8 and 26 neutron energy groups (reference Table 1).

Table 1. 8- and 26-neutron energy group upper energy boundaries.

\begin{tabular}{ccc}
\hline $\begin{array}{c}\text { Energy } \\
\text { Group }\end{array}$ & \multicolumn{2}{c}{ Upper Boundary (eV) } \\
26-Group Structure & 8-Group Structure \\
\hline 1 & $1.964 \mathrm{E}+07$ & $1.964 \mathrm{E}+07$ \\
2 & $6.703 \mathrm{E}+06$ & $7.065 \mathrm{E}+05$ \\
3 & $3.329 \mathrm{E}+06$ & $4.097 \mathrm{E}+03$ \\
4 & $5.784 \mathrm{E}+05$ & $4.000 \mathrm{E}+00$ \\
5 & $9.466 \mathrm{E}+04$ & $6.250 \mathrm{E}-01$ \\
6 & $1.858 \mathrm{E}+04$ & $2.800 \mathrm{E}-01$ \\
7 & $2.996 \mathrm{E}+03$ & $1.380 \mathrm{E}-01$ \\
8 & $1.344 \mathrm{E}+03$ & $5.550 \mathrm{E}-02$ \\
9 & $6.773 \mathrm{E}+02$ & \\
10 & $2.683 \mathrm{E}+02$ & \\
11 & $1.262 \mathrm{E}+02$ & \\
12 & $5.993 \mathrm{E}+01$ & \\
13 & $2.789 \mathrm{E}+01$ & \\
14 & $1.355 \mathrm{E}+01$ & \\
15 & $8.300 \mathrm{E}+00$ & \\
16 & $4.933 \mathrm{E}+00$ & \\
17 & $2.330 \mathrm{E}+00$ & \\
18 & $1.251 \mathrm{E}+00$ & \\
19 & $6.250 \mathrm{E}-01$ & \\
20 & $3.250 \mathrm{E}-01$ & \\
21 & $1.900 \mathrm{E}-01$ & \\
22 & $1.200 \mathrm{E}-01$ & \\
23 & $7.650 \mathrm{E}-02$ & \\
\hline
\end{tabular}


Table 1. (continued).

\begin{tabular}{|c|c|c|}
\hline \multirow{2}{*}{$\begin{array}{l}\text { Energy } \\
\text { Group }\end{array}$} & \multicolumn{2}{|c|}{ Upper Boundary (eV) } \\
\hline & 26-Group Structure & 8-Group Structure \\
\hline 24 & $4.730 \mathrm{E}-02$ & \\
\hline 25 & $1.483 \mathrm{E}-02$ & \\
\hline 26 & $7.145 \mathrm{E}-03$ & \\
\hline
\end{tabular}

The LOFC\#1 transient is initiated by reducing helium flow over a 10 second ramp down to zero flow. The control rods remain stationary, simulating an anticipated transient without scram, and the vessel cooling system remains operational for heat removal on the outside of the reactor vessel. The fission process is initially shutdown by an increase in fuel temperature and the resultant Doppler feedback. As the transient progresses, cooldown of the fuel and moderator and an eventual decrease in xenon concentration result in enough positive reactivity for the reactor to obtain re-criticality. Twenty hours of simulation time are being modeled. There are two main areas of interest for this transient: (1) the initial phase where flow is lost and the reactor becomes sub-critical and (2) the phase where the reactor attains re-criticality.

A summary of the testing matrix for the adaptive time-step methods is shown in Table 2. The techniques and tolerances included in the testing matrix were recommended by the developer of the adaptive time-step method based on his expertise of the methods and knowledge of the HTTR model (i.e., not all options included in Appendix B were tested). The main figure of merit for this transient is fission power; however, fuel temperature in a fixed location (ring 1 axial center) was also selected for comparison to check the effect of these changes on temperature. These parameters are compared using the various methods in PHISICS/RELAP5-3D and the relative differences (in percent) to the non-time enhanced case (reference) are shown.

Table 2. Adaptive time-step testing matrix.

\begin{tabular}{|l|l|l|}
\hline \multicolumn{1}{|c|}{ Method 1 } & \multicolumn{1}{c|}{ Method 2 } & \multicolumn{1}{c|}{ Method 3 } \\
\hline Flux_toll (tolerance $=1 \mathrm{E}-5)$ & Flux_toll (tolerance =1E-5) & Flux_toll (tolerance = 1E-5) \\
\hline Flux1_toll (tolerance $=1 \mathrm{E}-4)$ & Flux1_toll (tolerance = 1E-4) & Flux1_toll (tolerance = 1E-4) \\
\hline Flux_toll (tolerance $=1 \mathrm{E}-6)$ & $\begin{array}{l}\text { Maximum time step calculated } \\
\text { by flux_toll and flux1_toll }\end{array}$ & \\
\hline Flux_toll (tolerance $=$ 1E-4) & & \\
\hline Flux1_toll (tolerance $=1 \mathrm{E}-3)$ & & \\
\hline Flux1_toll $($ tolerance $=1 \mathrm{E}-5)$ & & \\
\hline
\end{tabular}

\section{TEST RESULTS}

The methods in Table 2 were tested on the HTTR PHISICS/RELAP5-3D model for both the 8- and 26-neutron energy group calculations. The thermal hydraulic portion of the model is the same for these two cases. For each calculation using the adaptive time-step method, a minimum and maximum time step of $1.0 \mathrm{~s}$ and $10.0 \mathrm{~s}$ was used for the first hour of the transient and a minimum and maximum time step of $2.0 \mathrm{~s}$ and $10.0 \mathrm{~s}$ was used for the remainder of the transient. For the reference run, a constant $1.0 \mathrm{~s}$ time step for both thermal hydraulic and neutron kinetic was used for the first hour of the transient, and a constant $2.0 \mathrm{~s}$ time step was used for the rest of the transient. The 8-group results are presented in Section 4.1 and the 26-group results in Section 4.2. 


\subsection{HTTR Model Using 8 Neutron Energy Groups}

\subsubsection{Methods 1, 2, and 3 Using "flux_toll" Option}

Using the "flux_toll" option for each of the three adaptive time-step methods, a review of reactor fission power, Ring 1 midline fuel temperature, and a relative percent difference from the reference method are provided. (The reference method uses no adaptive time steps [i.e., the approach up to now].)

Figure 1 and Figure 2 show power and fuel temperature behaviors, respectively, for each of the three methods, and the reference calculation. Only one line is seen in these figures, as a result of the data overlapping very closely. The timing of the re-critical peak around 12 hours after the LOFC started matches up very well to the reference case for each method; and on the scale shown here, the magnitude seems to be very similar as well.

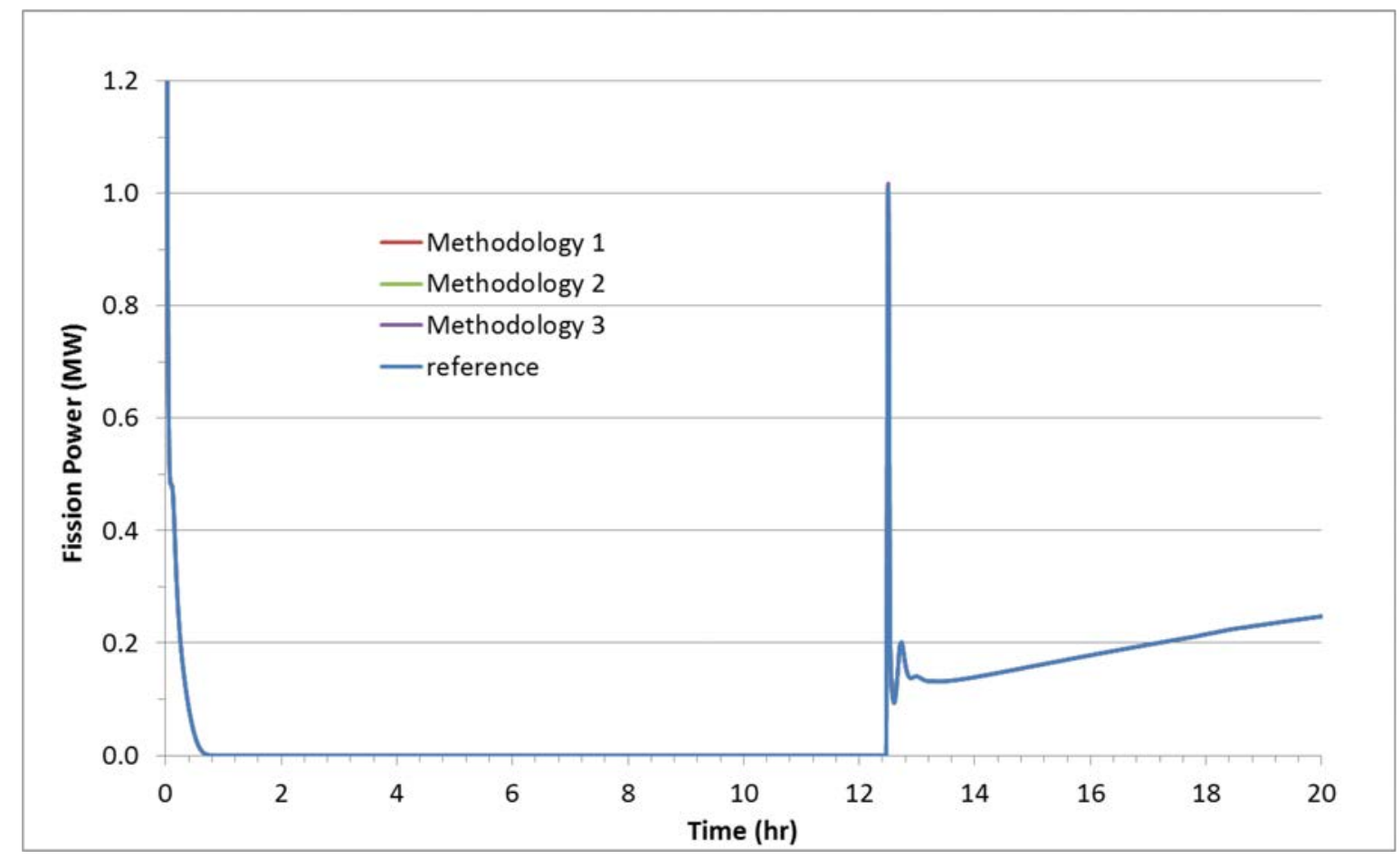

Figure 1. Fission power (MW) for the reference case and Methods 1, 2, and 3 using flux_toll with a tolerance of $1 \mathrm{E}-5$. 


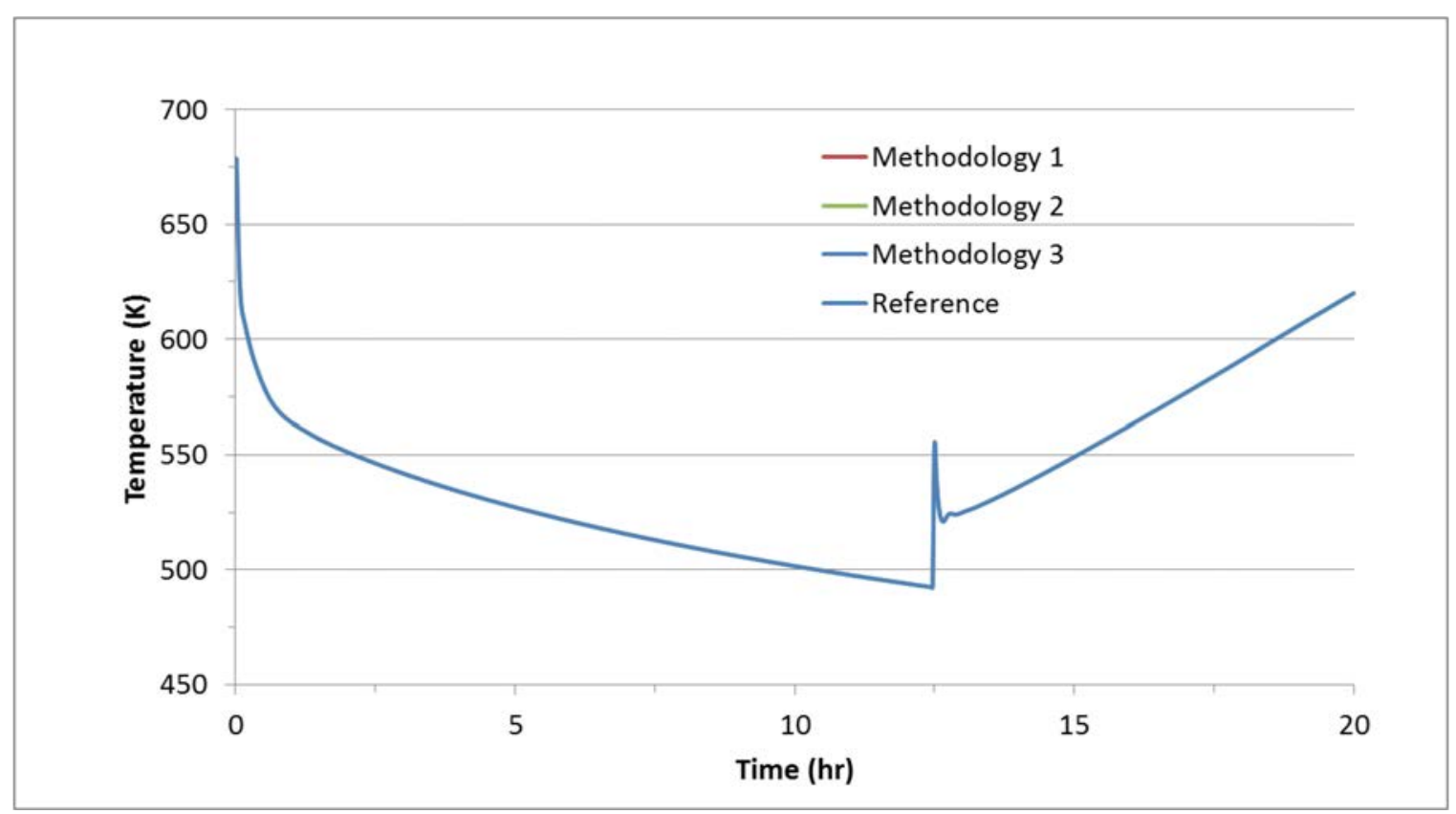

Figure 2. Ring 1 fuel block average temperature $(\mathrm{K})$ at the approximate axial mid-point for the reference case and Methods 1, 2, and 3 using flux_toll with a tolerance of 1E-5.

Figure 3 shows the fission power difference $(\mathrm{kW})$ from the reference case for the three methods using the flux_toll option with a tolerance set at 1E-5. Both the timing and magnitude of the re-critical peak result in differences from the reference case. Various options for normalizing the differences between these time-dependent data sets are available. In this report, all relative fission power percentage differences are normalized to the steady-state reactor fission power, which is very close to $9 \mathrm{MW}$. Only the absolute value is shown for clarity (i.e., all differences are positive).

Figure 4 indicates that during the re-criticality phase, Method 1 produced the highest difference at about $6.0 \%$ (i.e., approximately $600 \mathrm{~kW}$ ). 


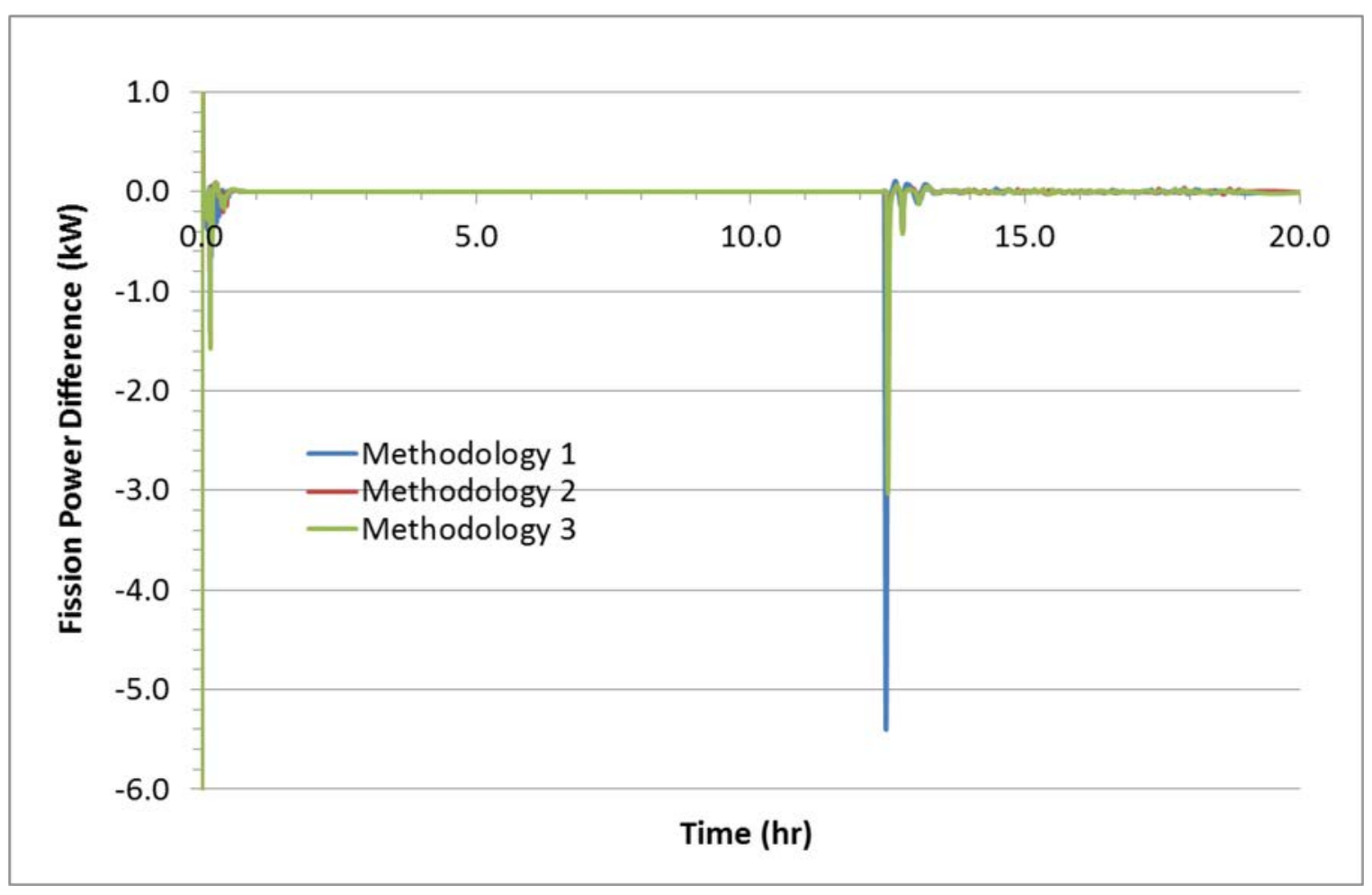

Figure 3. Fission power difference $(\mathrm{kW})$ between the reference case and Methods 1, 2, and 3 using flux_toll with a tolerance of $1 \mathrm{E}-5$.

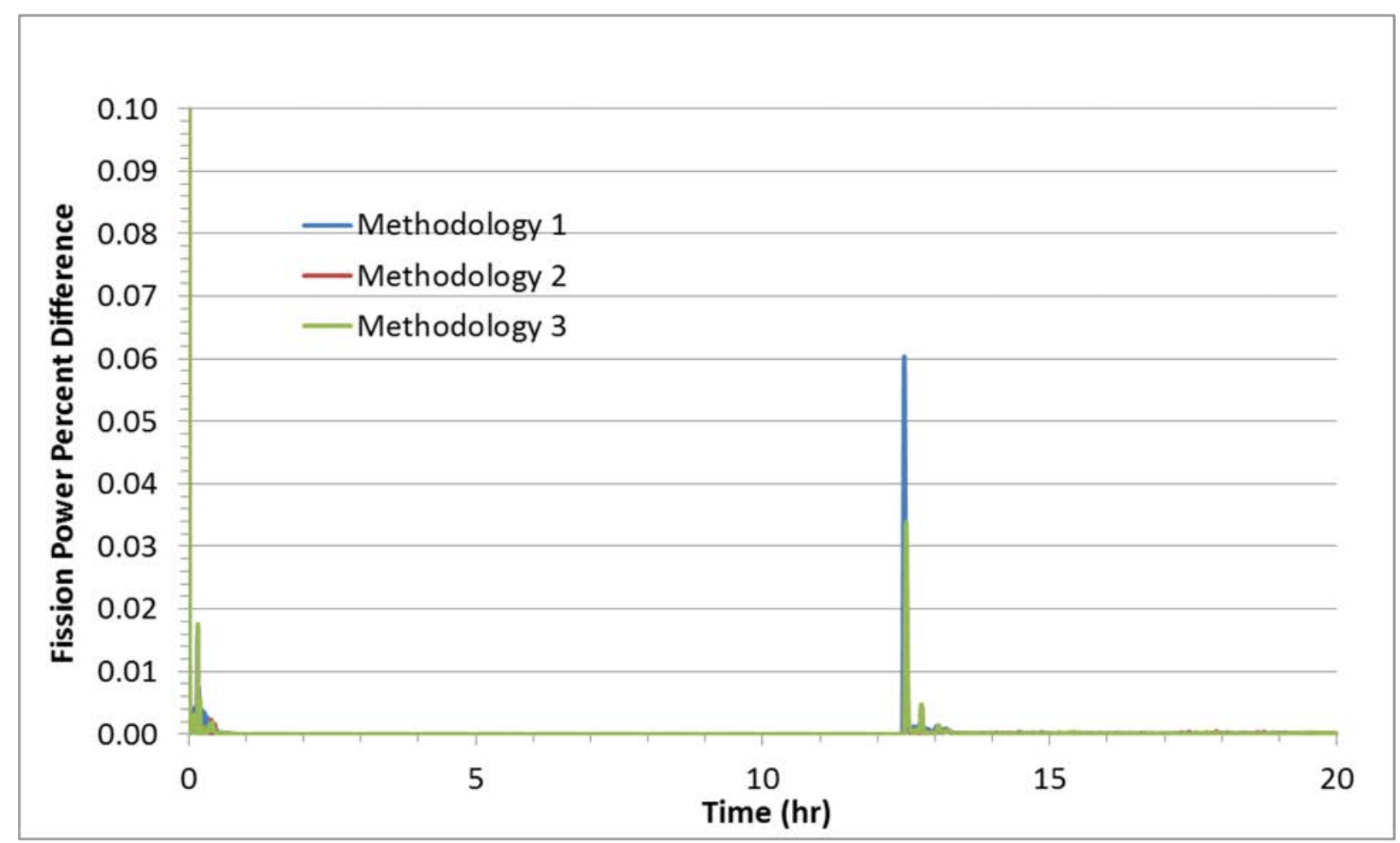

Figure 4. Fission power percent difference normalized to the steady state fission power between the reference case and Methods 1, 2, and 3 using flux_toll with a tolerance of 1E-5. 
Figure 5 and Figure 6 are focused on the two main areas of interest. The initial phase of the transient has a very low error for all methods, with Method 2 being the best performing at less than $0.35 \%$. For the re-critical phase (Figure 6), the difference results from the rate at which the power rises due to recriticality, as opposed to the actual magnitude of the peak being significantly different. At 12.5 hours, the power peak is reached, and the percent difference for the three methods at that time is less than $0.06 \%$.

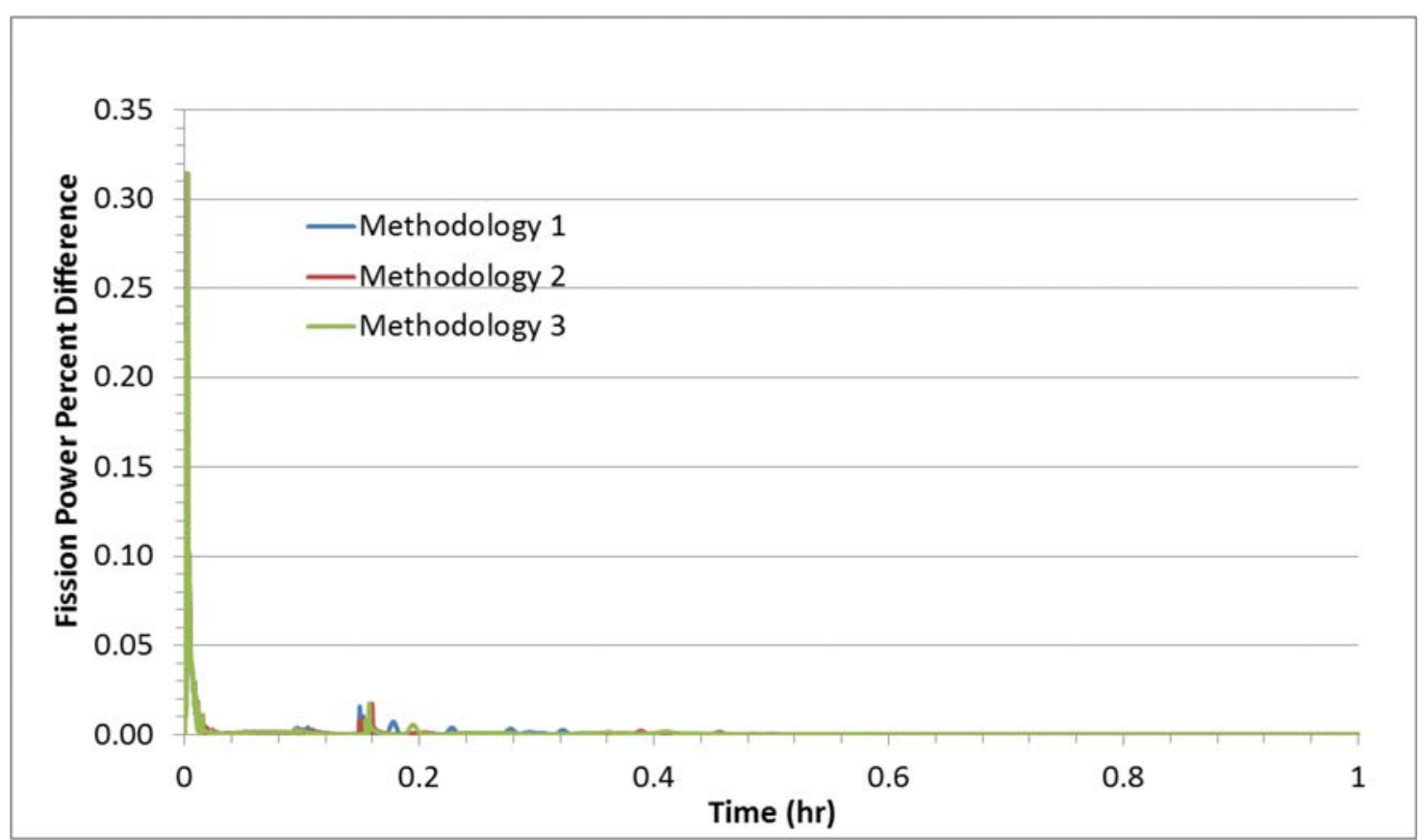

Figure 5. Fission power percent difference in the first 1.8 hours between the reference case and Methods 1,2 , and 3 using flux_toll with a tolerance of 1E-5.

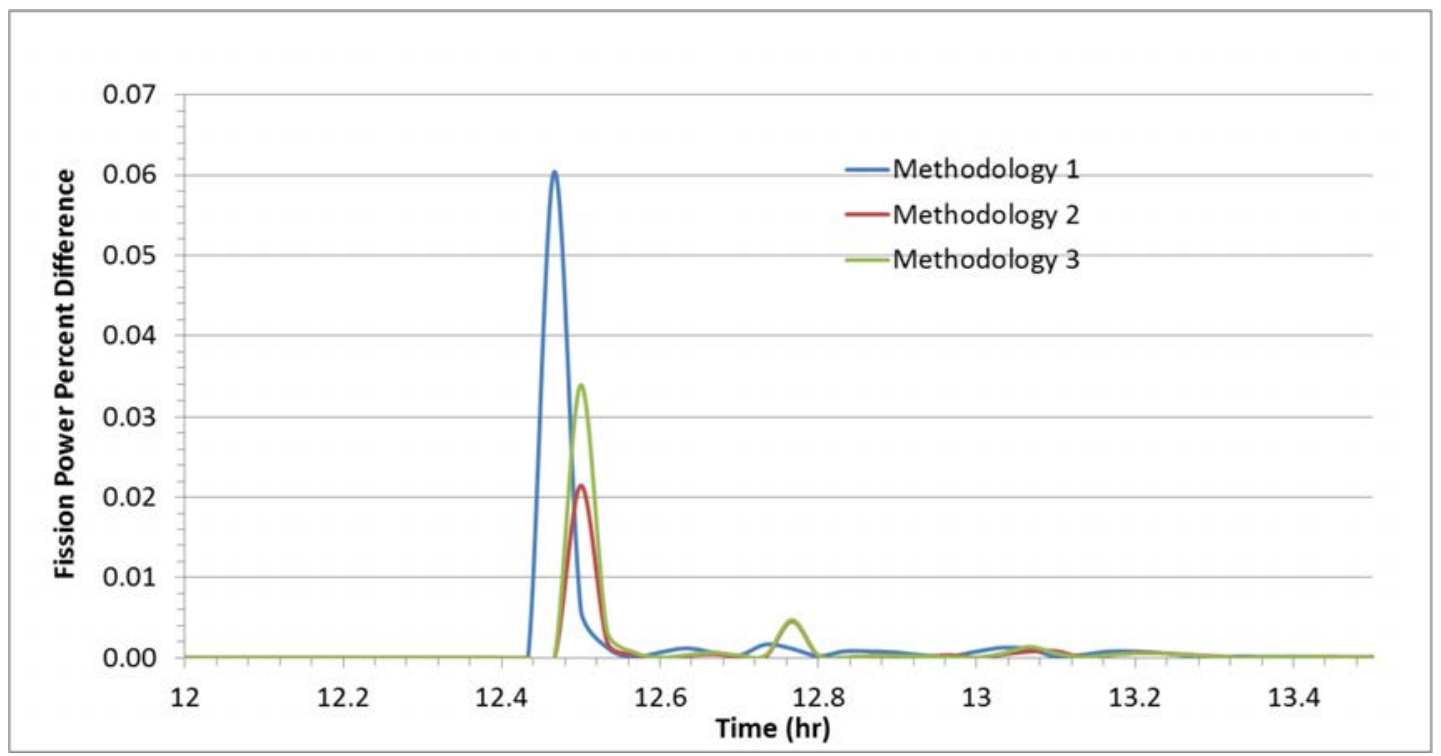

Figure 6. Fission power percent difference in the re-criticality period between the reference case and Methods 1, 2, and 3 using flux_toll with a tolerance of 1E-5. 
A review of the fuel temperature for the three methods is provided in Figure 7. The particular location of the chosen heat structure is the third fuel block down from the top of the core in the inner fuel ring 1. The fuel temperature percentage differences are normalized to the reference case initial temperature in the ring 1 mid-plane location, following the same philosophy as the fission power normalization. The differences are insignificant for all three methods (less than $0.01 \%$ or $0.1 \mathrm{~K}$ ), which is well below the RELAP5-3D model resolution. Therefore, for all practical purposes, the very small differences noted in fission power did not make a noticeable impact on fuel temperatures.

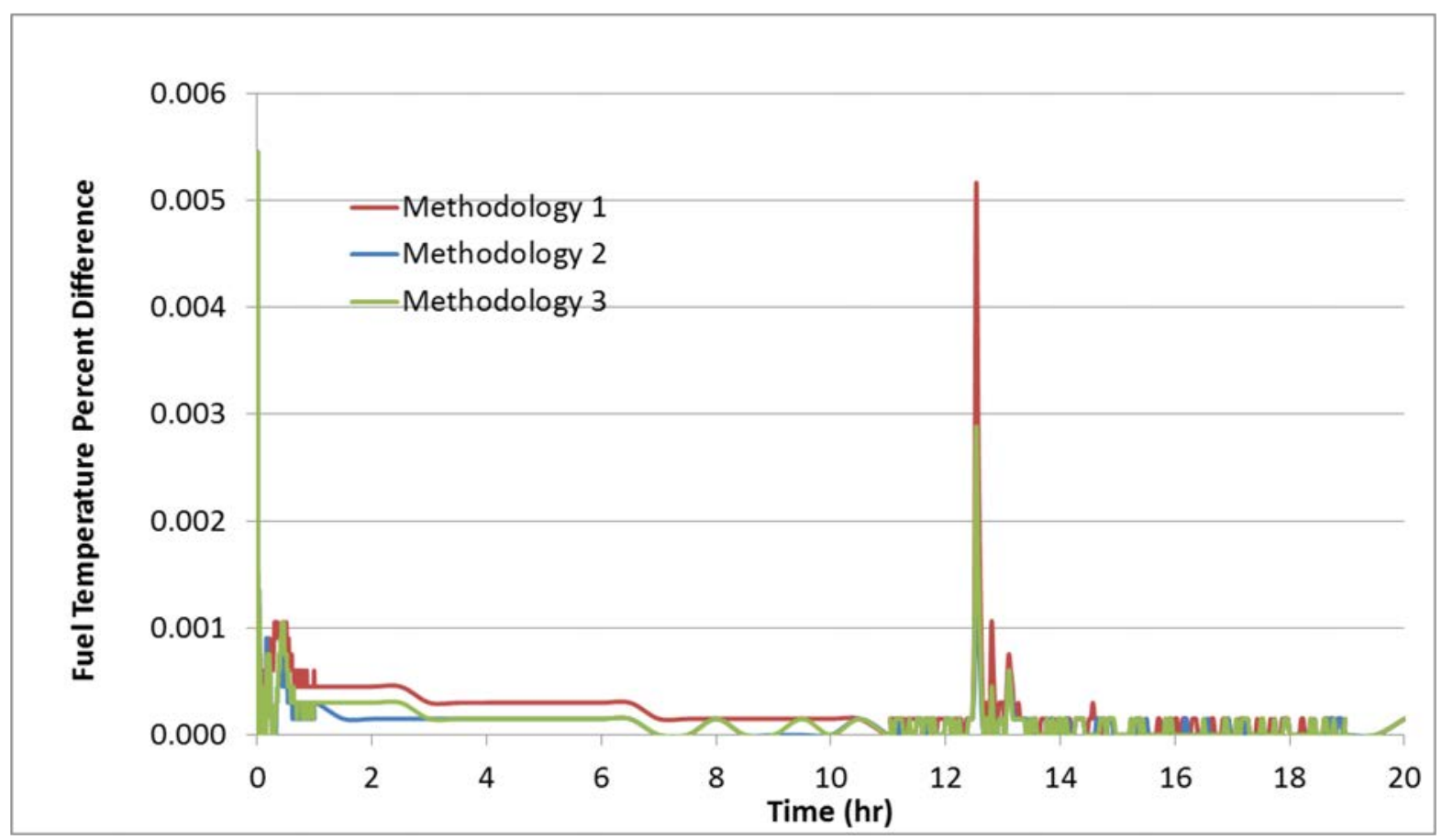

Figure 7. Fuel temperatures percent difference comparison between the reference to Methods 1, 2, and 3 using flux_toll with a tolerance of 1E-5.

All calculations were performed using the Falcon cluster on the INL HPC using 24 processors on one node. Calculation time savings achieved using Methods 1, 2, and 3 with flux_toll and a tolerance of 1E-5 is depicted in Figure 8. The actual problem time was plotted against a ratio of the central processing unit (cpu) (or real) time to the problem time. The lower the cpu-to-problem time ratio, the faster the calculation is performed. A ratio of 1.0 would represent a real-time simulation and a factor of eight on this plot implies the problem was simulated eight times slower than real-time.

It can be seen from Figure 8 that the performance of the adaptive time solver scheme varies over the transient, because it adjusts to changes in flux and temperatures by using smaller or larger time steps. The first hour of the simulation is the most expensive at a ratio of seven to eight times slower than real time, caused by the small time steps required to resolve the sharp decrease in power and gas flow in the core. After this first phase the simulation proceeds faster and reaches a minimum of 3 times slower than real time just before the start of the re-criticality phase at 12 hours.

In addition, it is apparent that none of the three methods resulted in significant improvement in cpu time. As expected, the reference run took the longest to complete. The total cpu time for the reference calculation was about 71 hours, while Methods 2 and 3 finished within 5 minutes of each other taking about 67 hours (see Table 3). Method 1 finished the simulation about 2.3 hours ahead of Methods 2 and 3. The time savings between the reference run and Method 2 (i.e., the method with the lowest percent difference) was about 3.8 hours or $5.4 \%$. Although any time saving without a commensurate penalty in 
accuracy is desirable from a simulation point of view, use of this method (flux_toll with tolerance 1E-05) only produced a minor benefit.

Table 3. Run time summary for Methods 1, 2 and 3 using flux_toll with a tolerance of 1E-5.

\begin{tabular}{lcc} 
& Test Case & $\begin{array}{r}\text { Total CPU Time (Hours) to Simulate } \\
\text { a 20 Hour LOFC Transient }\end{array}$ \\
\hline Reference & 70.87 \\
Method 1 & 64.68 \\
Method 2 & 67.02 \\
Method 3 & 66.90 \\
\hline
\end{tabular}

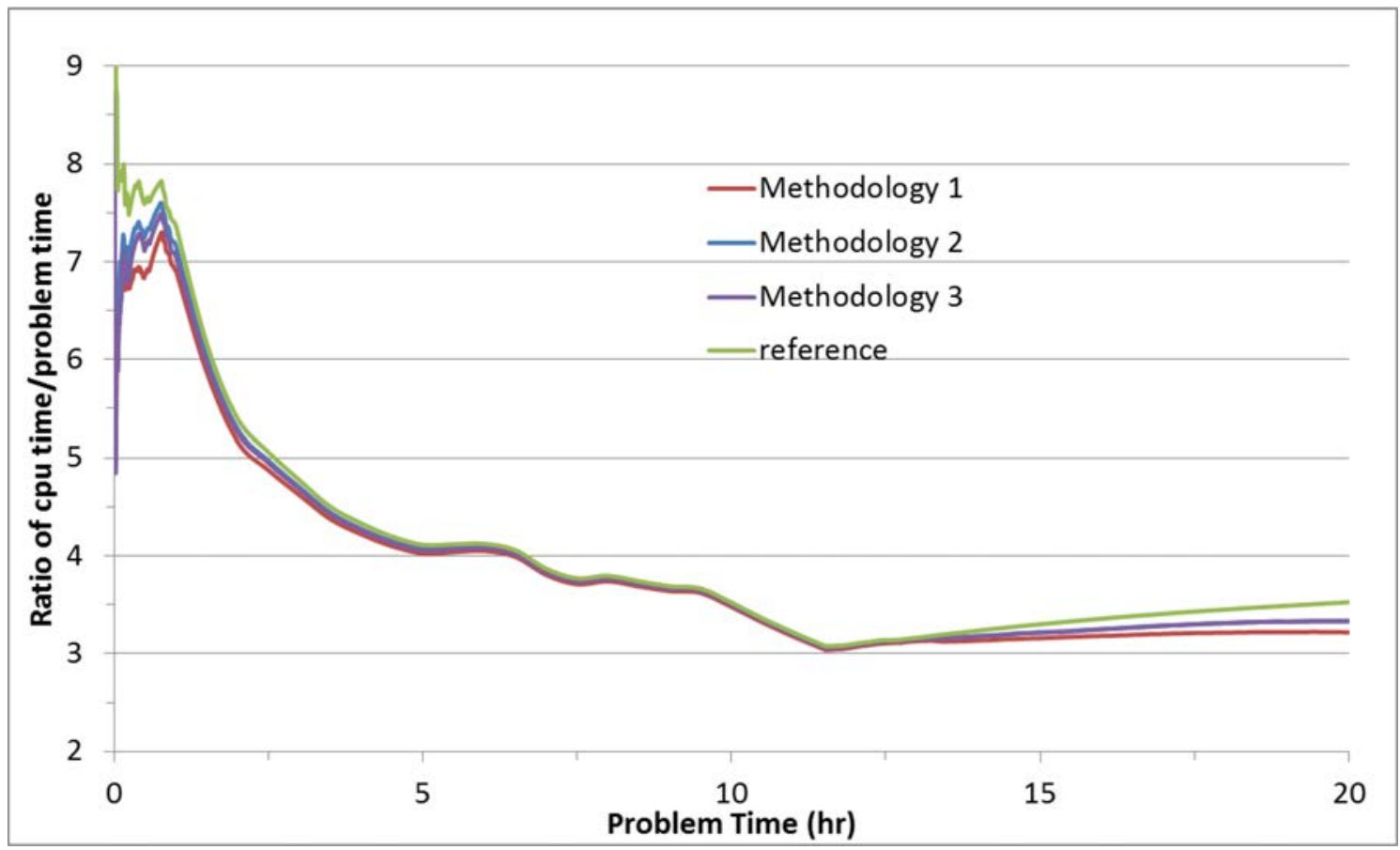

Figure 8. Time enhancement factor using a ratio of the cpu time to the problem time plotted over time for Methods 1, 2, and 3 using flux_toll with a tolerance of 1E-5.

\subsubsection{Method 1 using "flux_toll" Option with Additional Tolerance Values}

The tolerance factor plays an important role in adaptive time-step schemes. In this section, the effect of changing the tolerance, using Method 1 and the flux_toll option, by a factor of ten greater than and less than the value of 1E-5 used in Section 4.1.1 is investigated. A review of the effects of this tolerance change on accuracy and cpu time was performed. The three tolerance levels compared in this section for Method 1 using flux_toll are 1E-4, 1E-5, and 1E-6.

Similar to the observations made in Section 4.1.1, the fission power and fuel temperature plots shown in Figure 9 and Figure 10 indicate the data sets are very close to the reference case. Figure 11 and Figure 12 show that the least restrictive tolerance value of $1 \mathrm{E}-4$ resulted in a higher difference $(0.32 \%)$ than the most restrictive tolerance value of 1E-6 (0.05\%), which is what could be expected for specifying tighter tolerance criteria.

The cpu time difference associated with the three tolerance values is shown in Figure 13. The use of the least restrictive tolerance value of $1 \mathrm{E}-4$ resulted in the largest speed-up (i.e., $35 \%$ faster), because the 
solvers were allowed to take much larger time steps with this lower tolerance setting. The calculation is significantly accelerated in the first two hours and after the re-criticality has occurred. The cpu run times obtained for each tolerance are provided in Table 4. Although this improved time performance came at the expense of simulation accuracy, it should be noted that the difference is only $30 \mathrm{~kW}$ on a $1 \mathrm{MW}$ power peak in absolute terms. Fuel temperatures do not show any measurable variances at all, leading to the conclusion the 1E-4 tolerance setting could be quite useful for scoping studies when absolute accuracy is not important, but rapid model development and debugging is required. The use of a tighter tolerance than $1 \mathrm{E}-5$ is probably not recommended due to the minor improvement in run time achieved.

Table 4. Run time summary for Method 1 using flux_toll with tolerance values of 1E-4, 1E-5, and 1E-6.

\begin{tabular}{lc}
\multicolumn{1}{c}{ Test Case } & $\begin{array}{c}\text { Total CPU Time (Hours) to Simulate } \\
\text { a 20-Hour LOFC Transient }\end{array}$ \\
\hline Reference & 70.87 \\
Method 1 tolerance $=1 \mathrm{E}-4$ & 46.03 \\
Method 1 tolerance $=1 \mathrm{E}-5$ & 64.68 \\
Method 1 tolerance $=1 \mathrm{E}-6$ & 69.28 \\
\hline
\end{tabular}

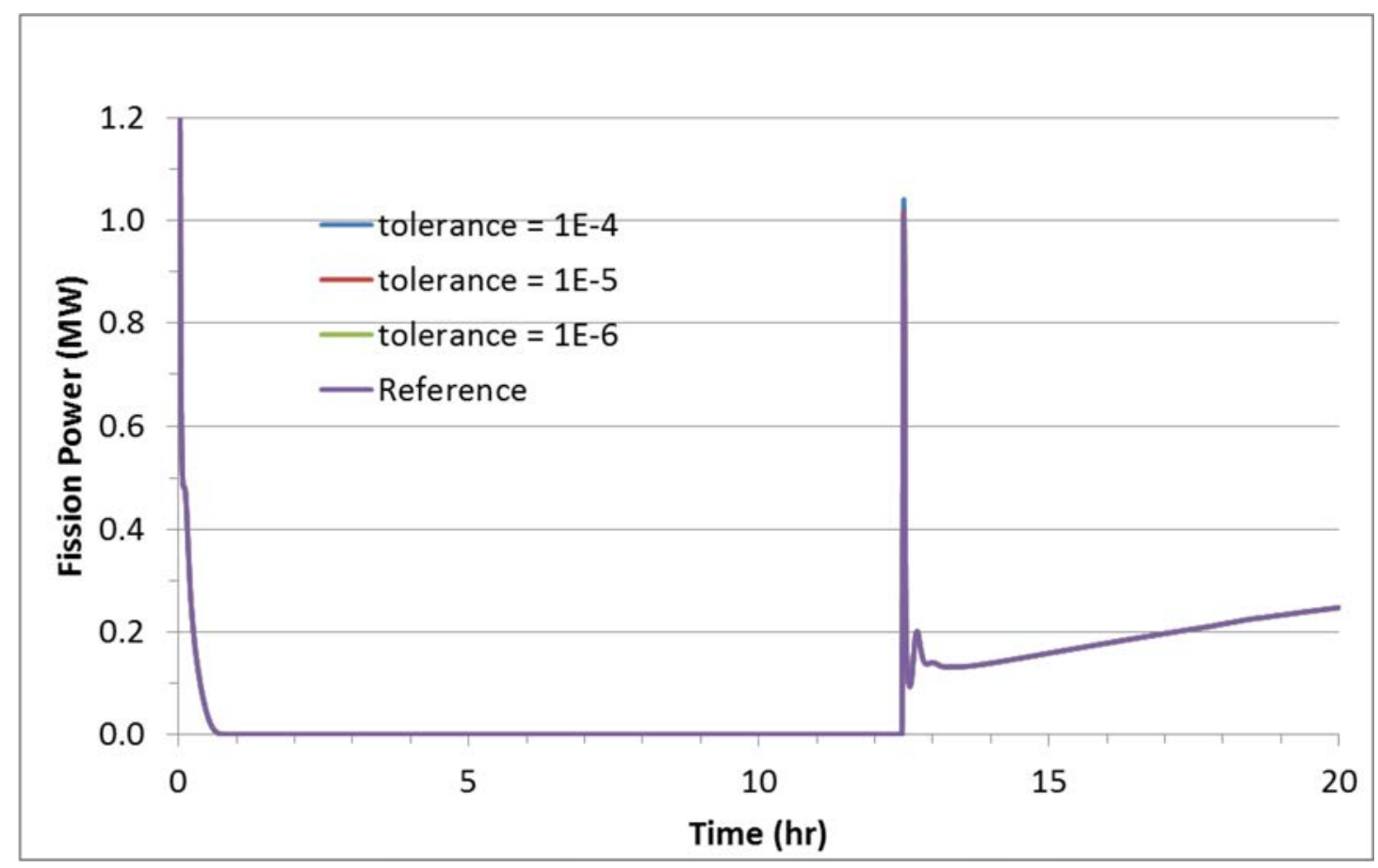

Figure 9. Fission power (MW) for the reference case and Method 1 flux_toll using three different tolerance values: $1 \mathrm{E}-4,1 \mathrm{E}-5$, and $1 \mathrm{E}-6$. 


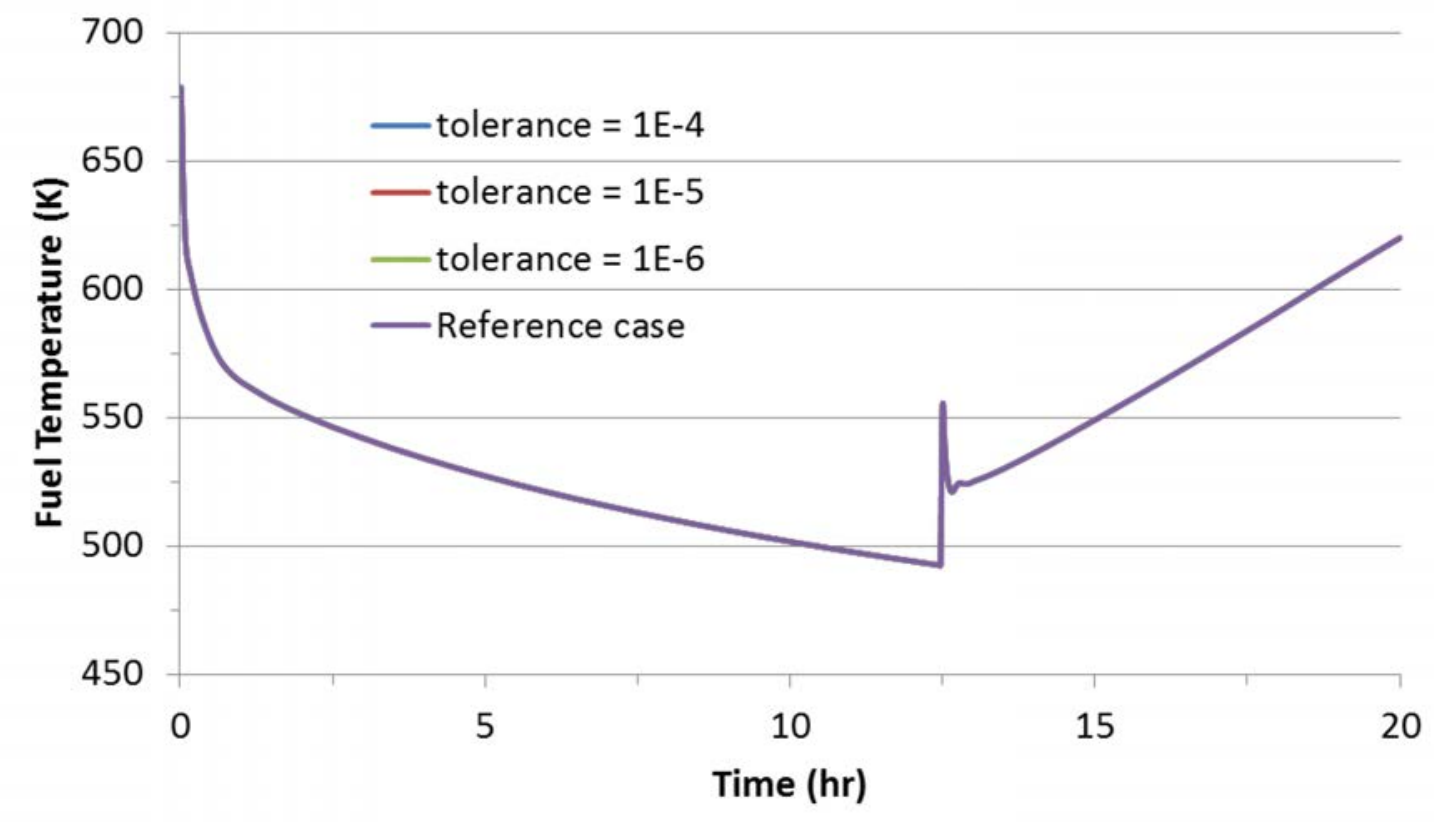

Figure 10. Ring 1 fuel block average temperature $(\mathrm{K})$ at the approximate axial mid-point for the reference case and Method 1 using flux_toll and three different tolerance values: $1 \mathrm{E}-4,1 \mathrm{E}-5$, and $1 \mathrm{E}-6$.

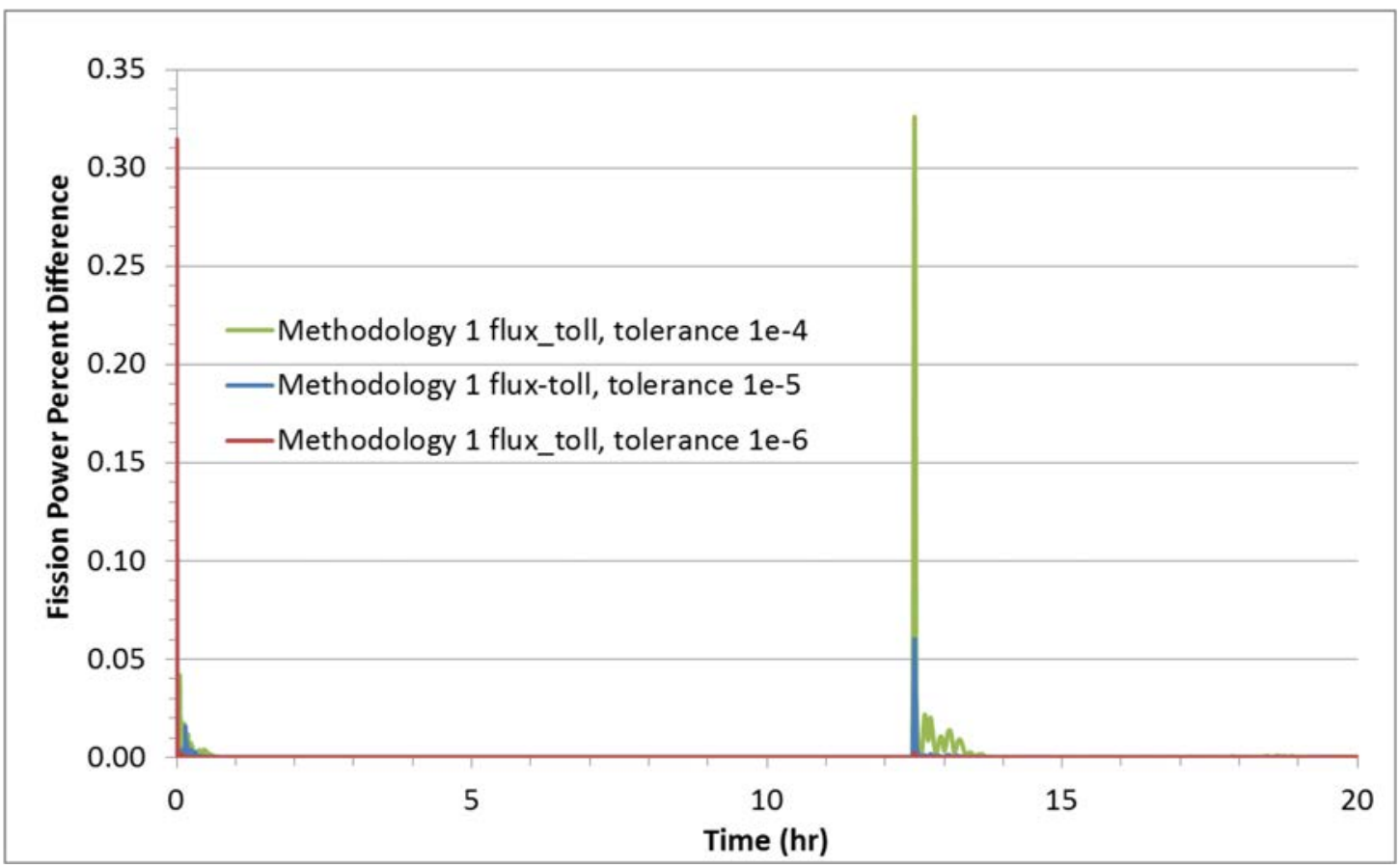

Figure 11. Fission power percent difference (from the reference case) for Method 1 using flux_toll with three different tolerance values: $1 \mathrm{E}-4,1 \mathrm{E}-5$, and $1 \mathrm{E}-6$. 


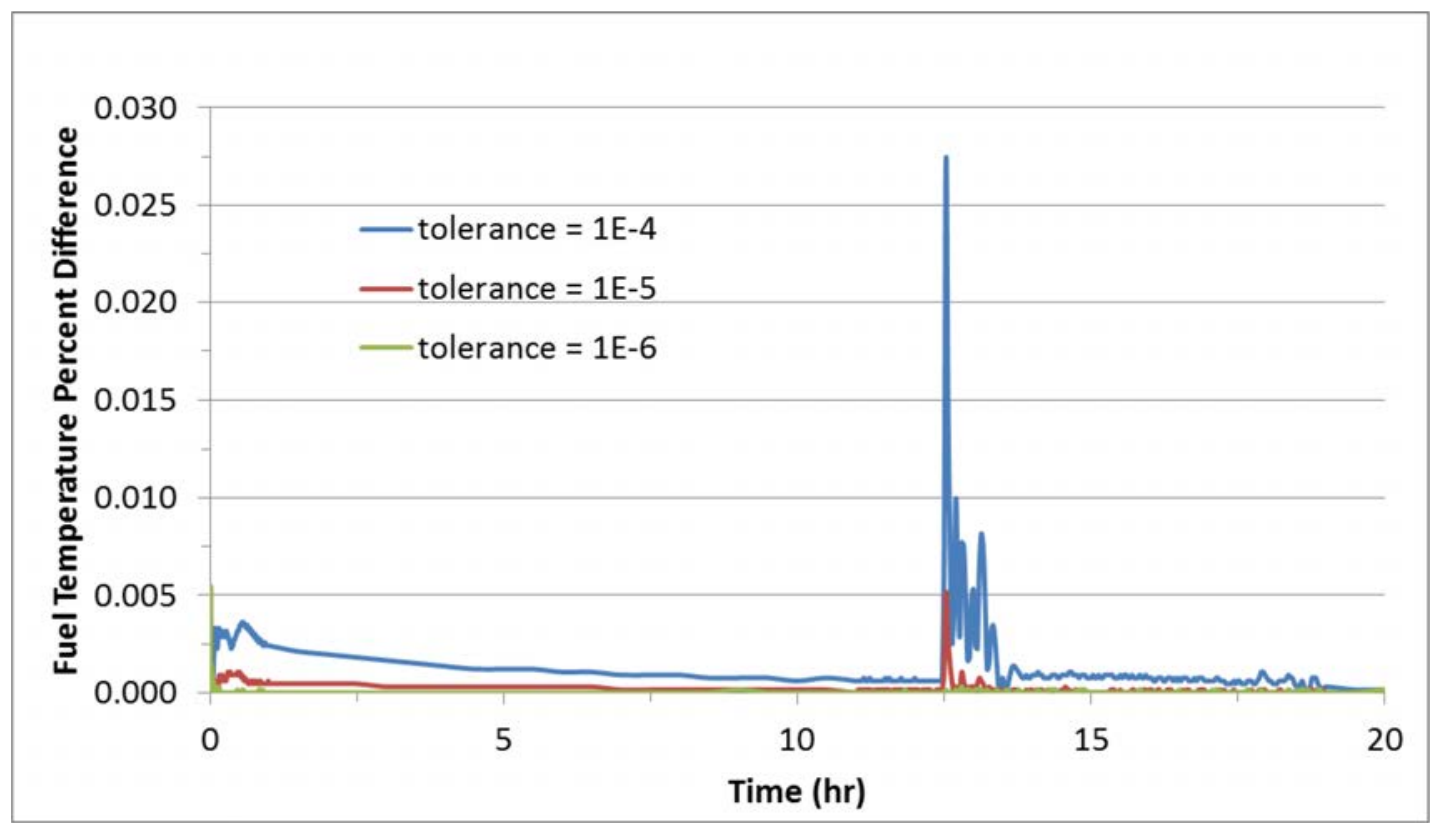

Figure 12. Fuel temperature percent difference (from the reference case) for Method 1 using flux_toll with three different tolerance values: $1 \mathrm{E}-4,1 \mathrm{E}-5$, and $1 \mathrm{E}-6$.

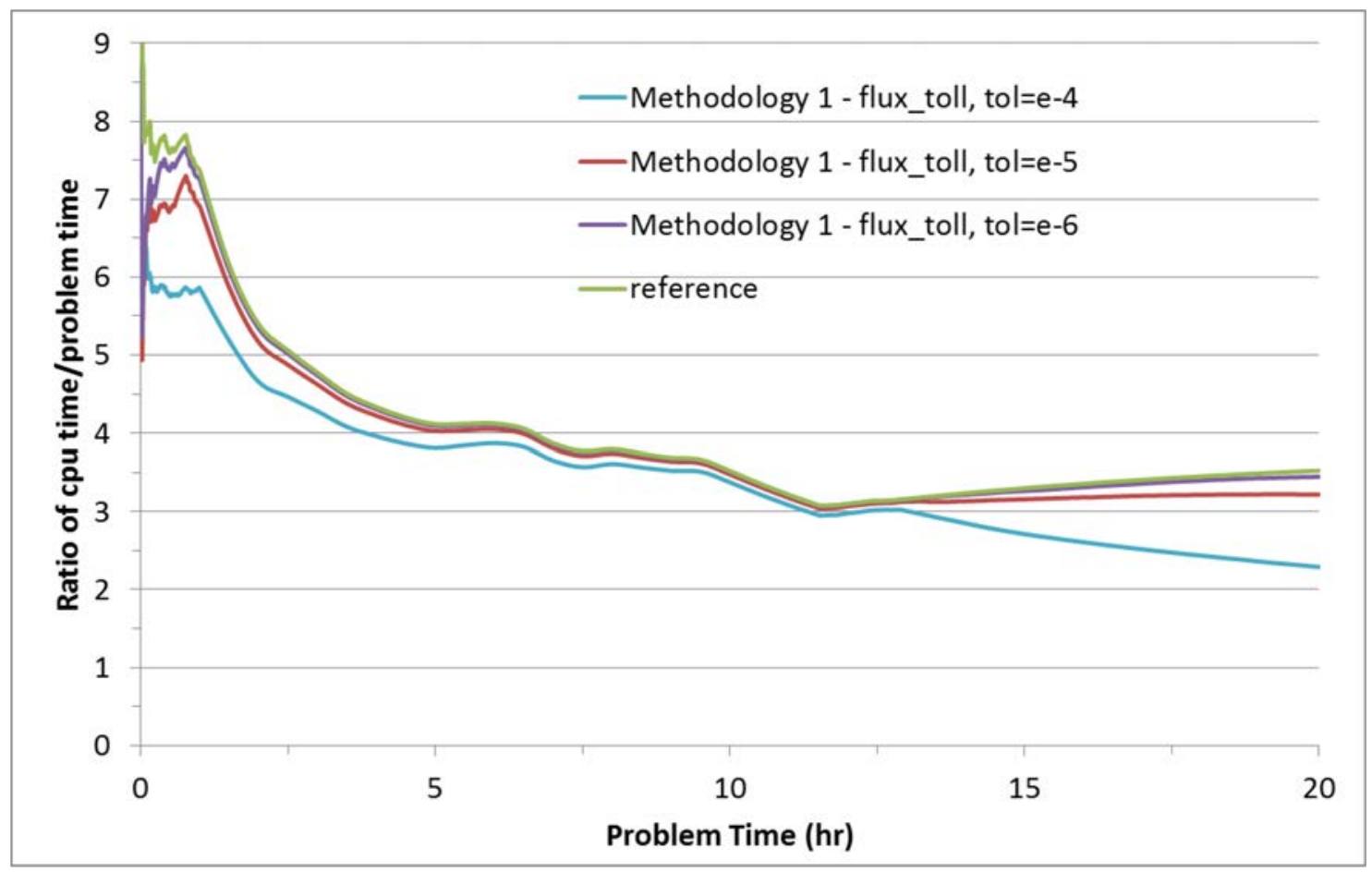

Figure 13. Time enhancement for the reference case and Method 1 using flux_toll and three different tolerance values: $1 \mathrm{E}-4,1 \mathrm{E}-5$, and $1 \mathrm{E}-6$. 


\subsubsection{Methods 1, 2, and 3 Using Flux1_toll}

In this section, the same comparisons are performed as in Section 4.1.1 for the flux_toll option, but now the flux1_toll option is used with the tolerance setting of 1E-4 (shown in Figure 14 through Figure 21). The plots show the overall trends first and then are separated into the areas of interest in order to highlight the results of the different methods.

The re-critical power peaks calculated using the flux1_toll option for each of the methods are offset from the reference by approximately 30 minutes (Figure 14). Additionally, Method 2 results in the calculation of a re-critical power peak that is about 12 times greater than the reference calculation and even above the $9 \mathrm{MW}$ steady-state power (Figure 17). This is clearly not an acceptable result. In contrast to this very large difference in power, the average fuel block temperatures for Method 2 are only about $35 \mathrm{~K}$ higher than the reference peak value of $555 \mathrm{~K}$ (Figure 19), again demonstrating that the large thermal heat capacity in the core graphite and the relative short duration of the fission power peak lead to a weak response in the fuel temperature. Method 3 produced low power and temperature differences with the reference case (Figure 17, Figure 18, Figure 20), and also led to a significant simulation time savings of about 53 hours or $75.3 \%$, as shown in Figure 21. After about 2.5 hours, the simulation using Method 3 proceeded at almost real-time or, in some sections, even faster than real-time.

The time enhancement achieved by Methods 1 and 2 are similar to Method 3 (i.e., the cpu time for the three methods was within 5 minutes of each other; see Table 5), but at the cost of significant accuracy loss in the case of Method 2 and, to a lesser degree, for Method 1 (i.e., compare Figure 18). Therefore, Method 3 is the recommended choice for use with this flux1_toll setting.

Table 5. Run time summary for Methods 1, 2 and 3 using flux1_toll with a tolerance of 1E-4.

\begin{tabular}{lcc} 
& Test Case & $\begin{array}{r}\text { Total CPU Time (Hours) to Simulate } \\
\text { a 20 Hour LOFC Transient }\end{array}$ \\
\hline Reference & 70.87 \\
Method 1 & 17.55 \\
Method 2 & 17.52 \\
Method 3 & 17.48 \\
\hline
\end{tabular}




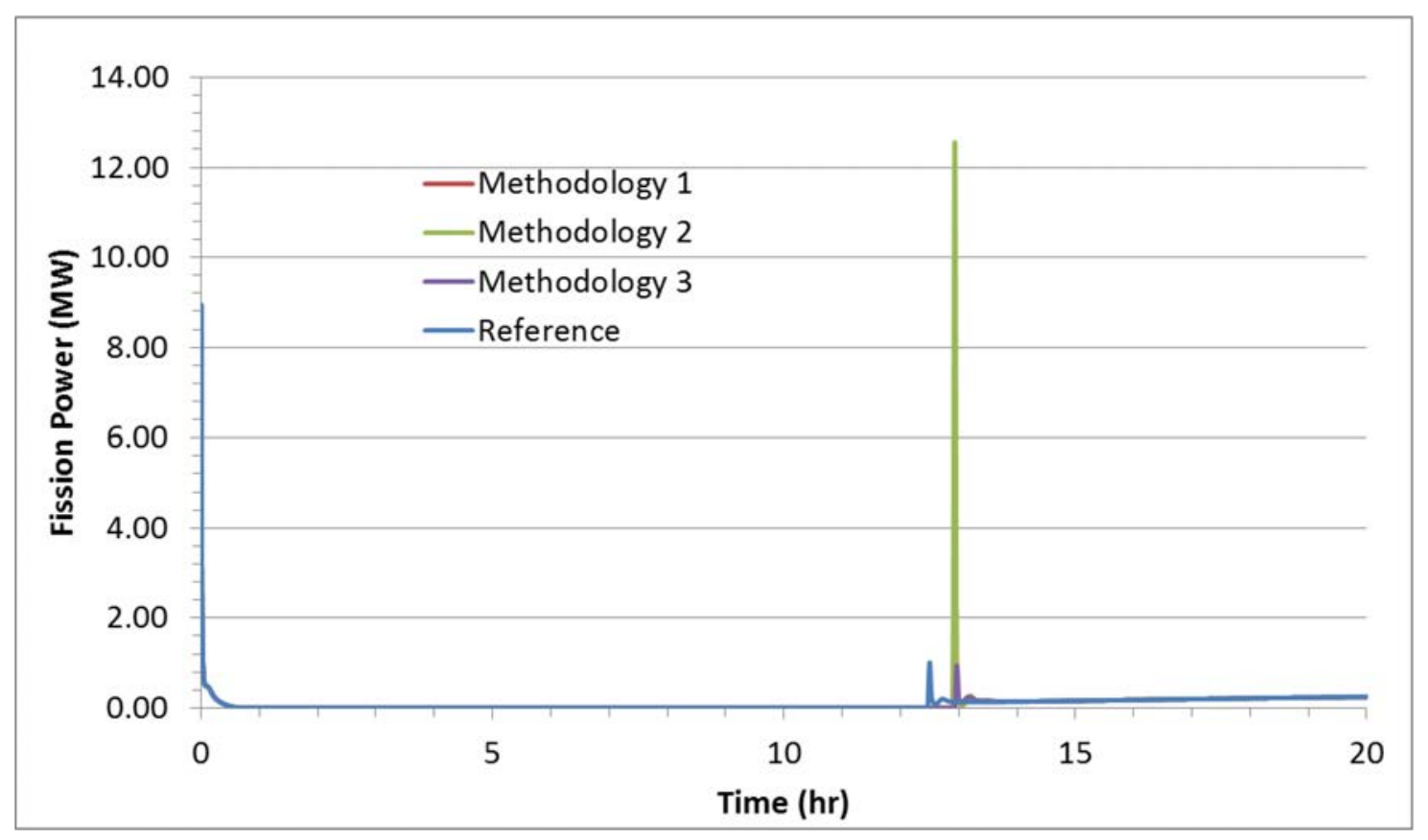

Figure 14. Fission power (MW) for the reference case and Methods 1, 2, and 3 using flux1_toll with a tolerance of $1 \mathrm{E}-4$.

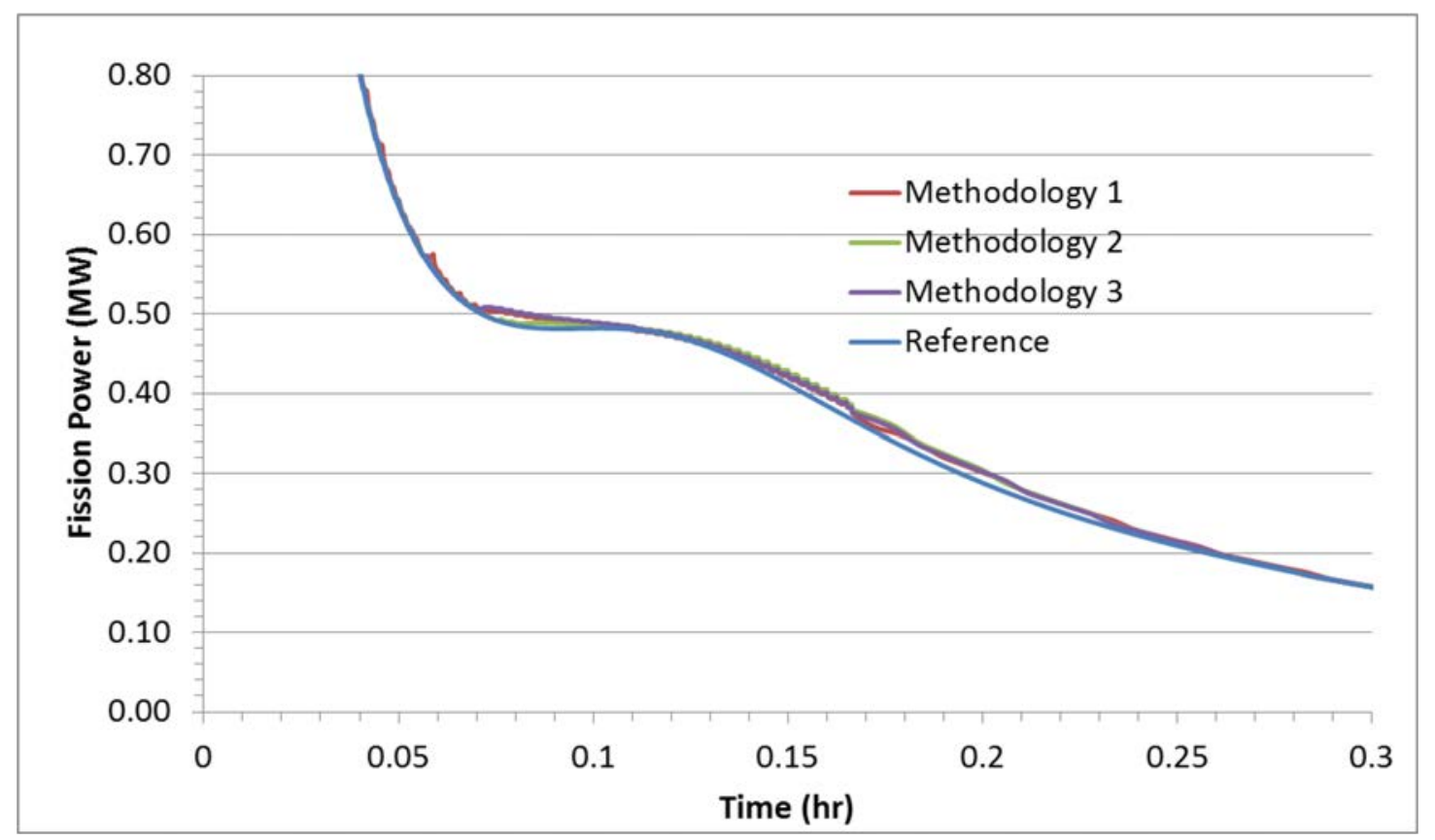

Figure 15. Fission power (MW) for the initial transient for the reference case and Methods 1, 2, and 3 using flux1_toll with a tolerance of $1 \mathrm{E}-4$. 


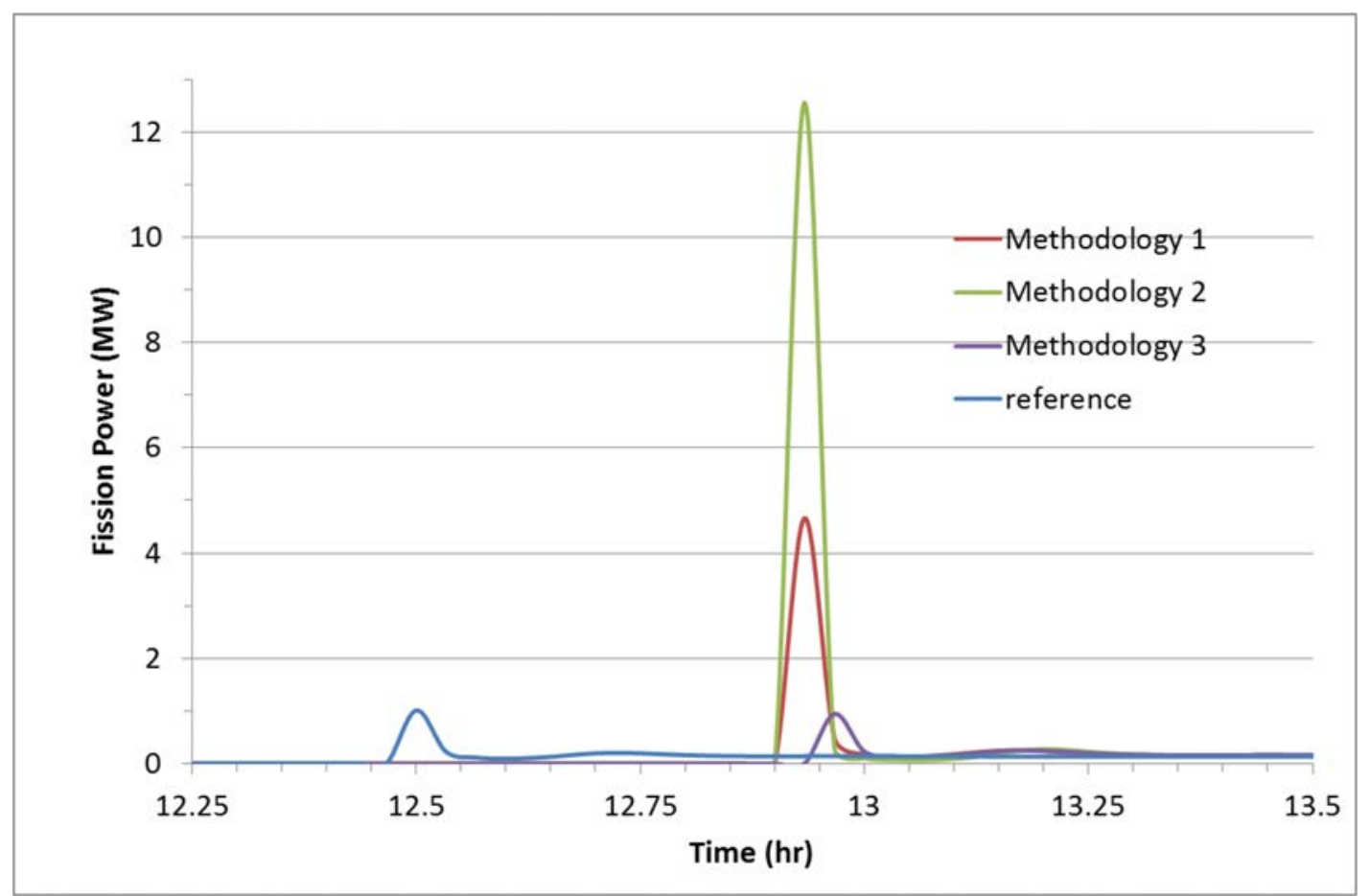

Figure 16. Fission power during the re-criticality portion of the transient for the reference case and Methods 1, 2, and 3 using flux1_toll with a tolerance of 1E-4.

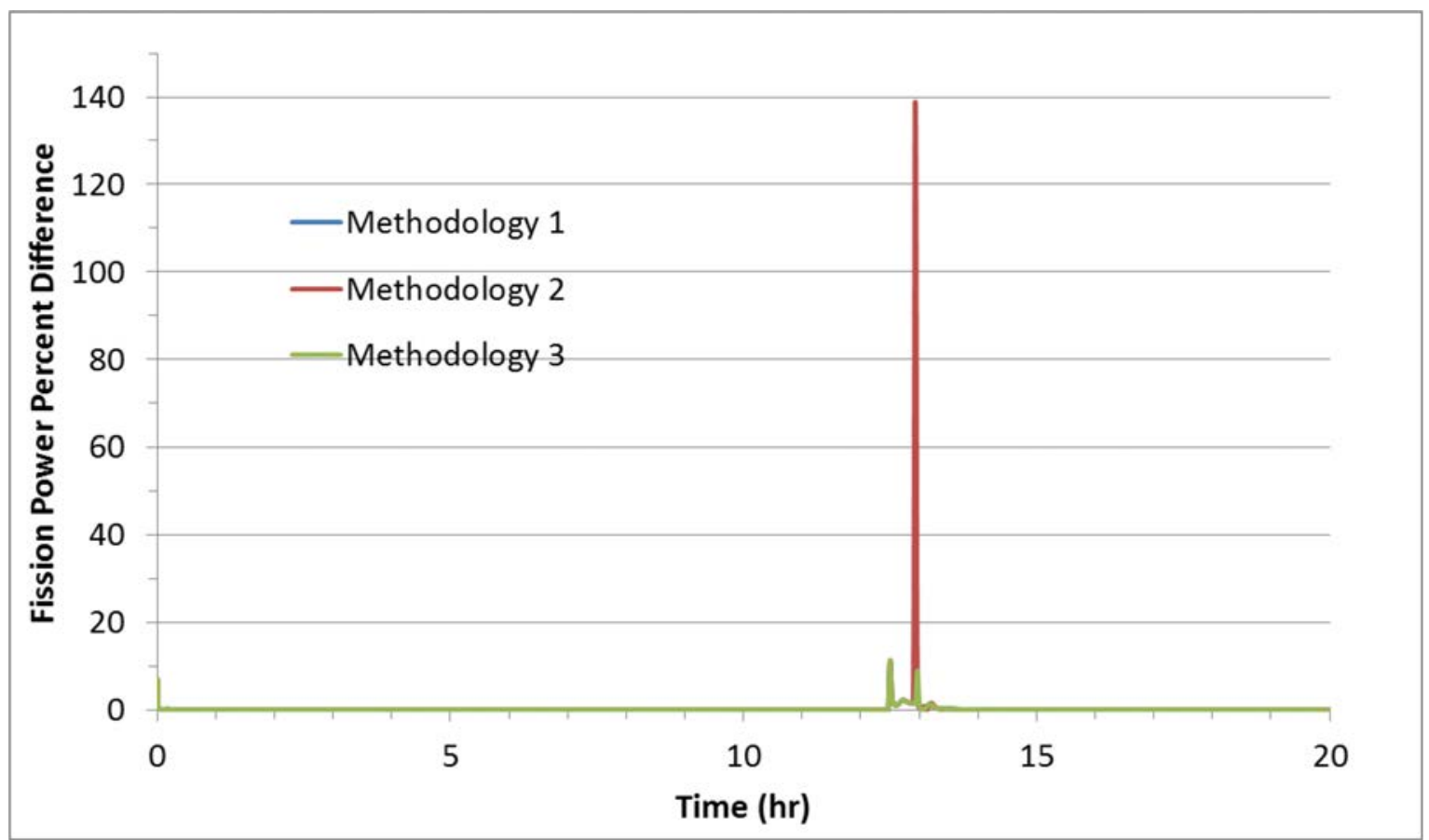

Figure 17. Fission power percent difference (from the reference case) for Methods 1, 2, and 3 using flux1_toll with a tolerance of $1 \mathrm{E}-4$. 


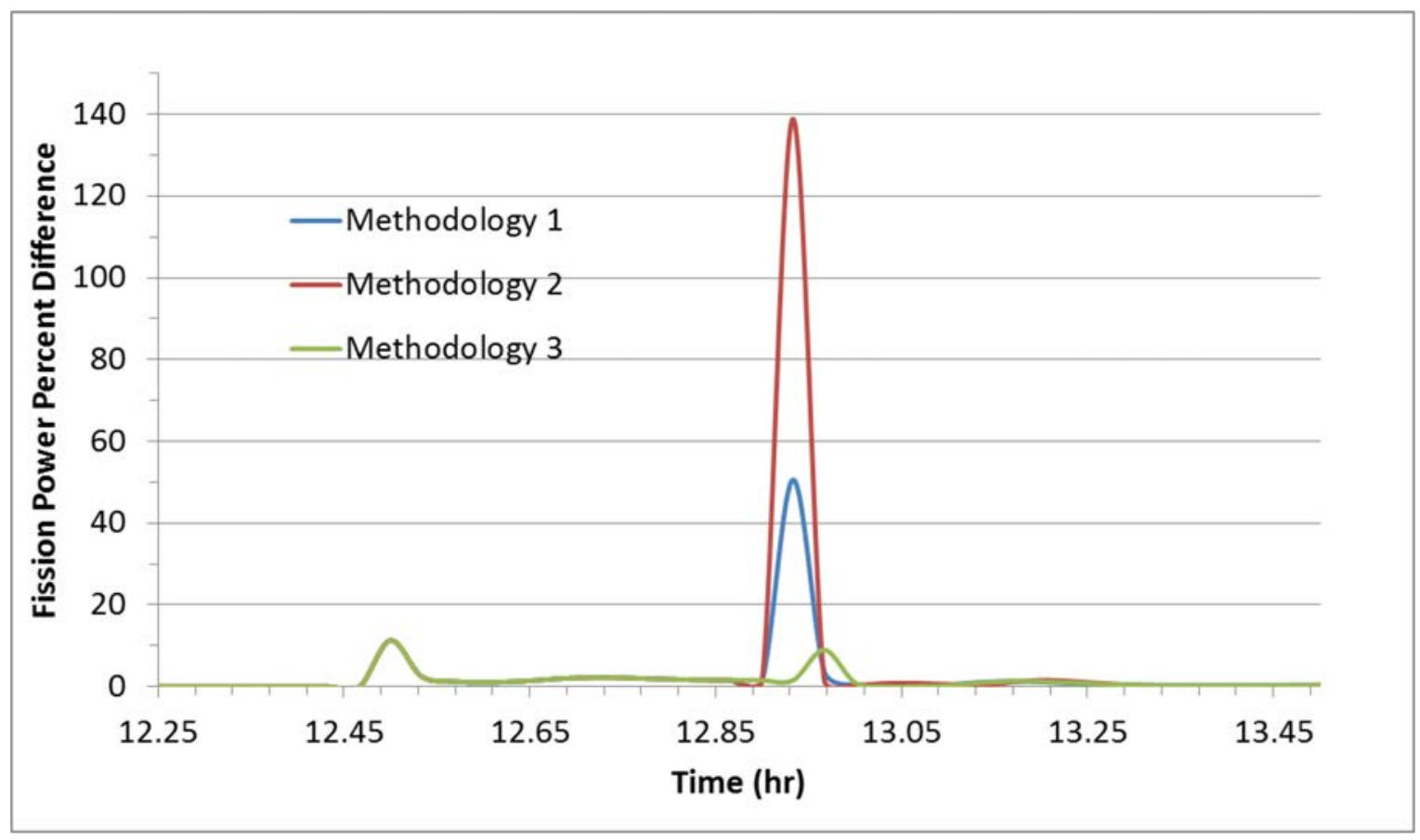

Figure 18. Fission power percent difference (from the reference case) in the re-criticality phase of the transient for Methods 1, 2, and 3 using flux1_toll with a tolerance of 1E-4.

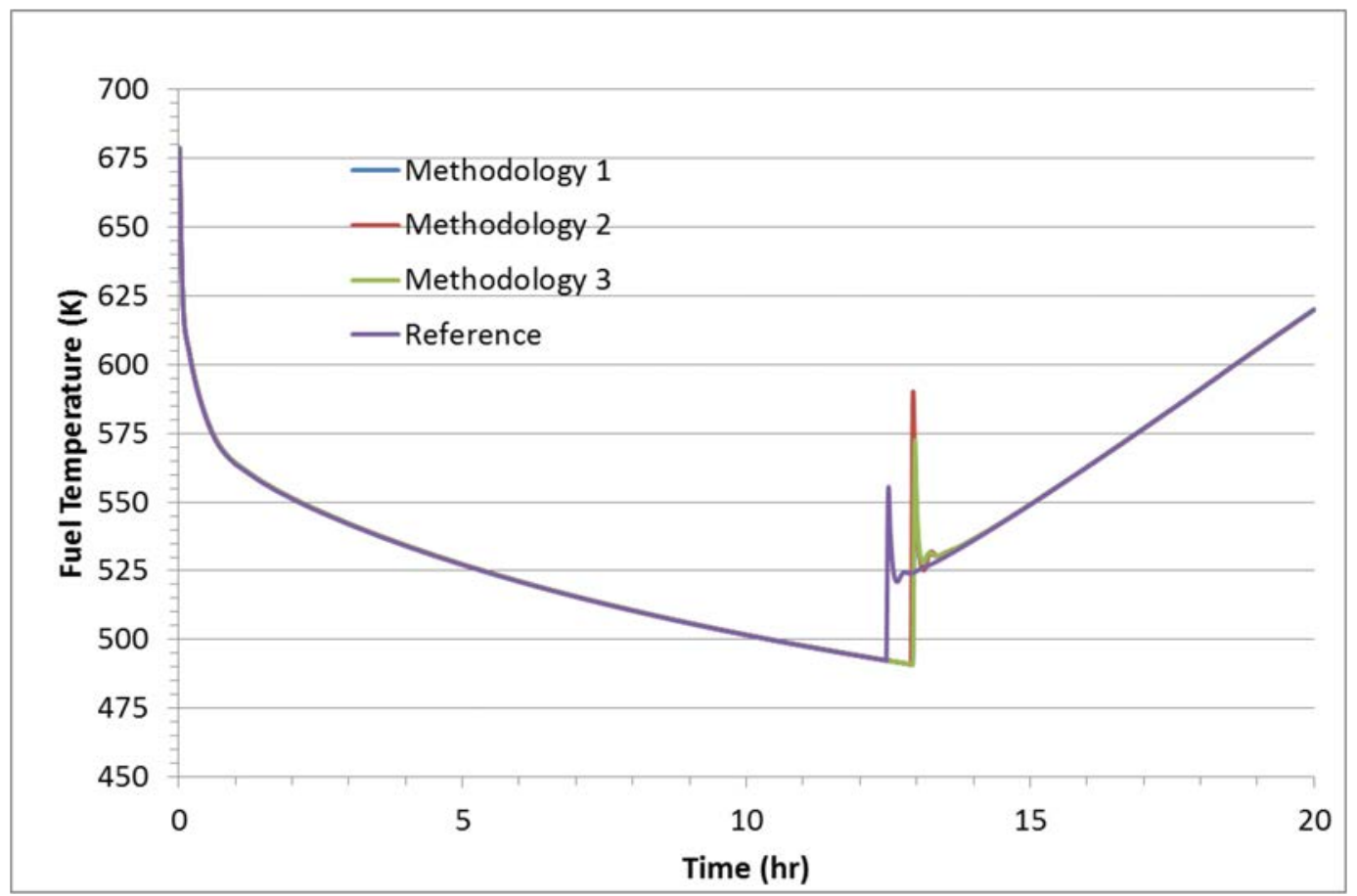

Figure 19. Ring 1 fuel block average temperature $(\mathrm{K})$ at the approximate axial mid-point for the reference case and Methods 1, 2, and 3 using flux1_toll with a tolerance of 1E-4. 


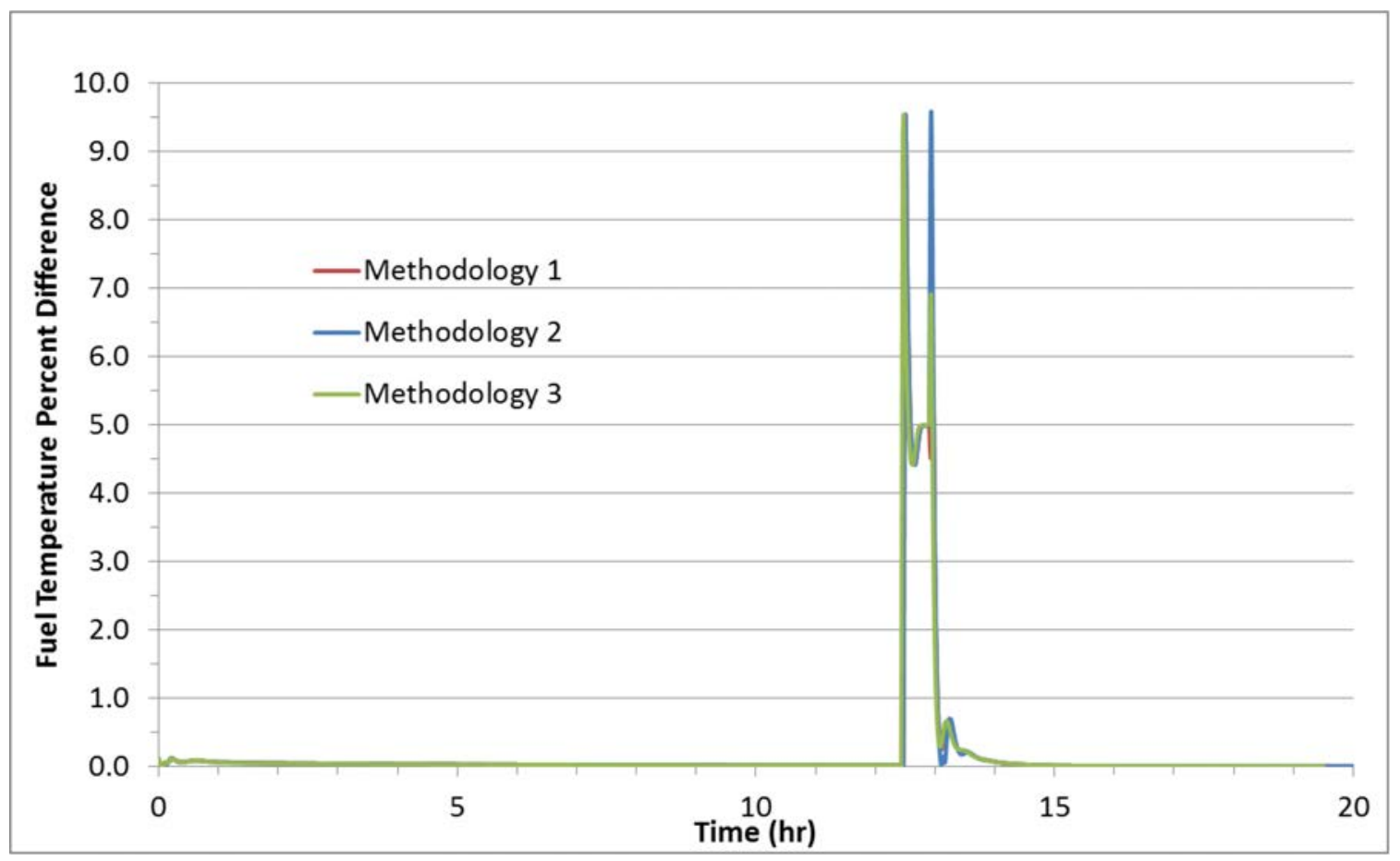

Figure 20. Fuel temperature percent difference (from the reference case) for Methods 1, 2, and 3 using flux1_toll with a tolerance of 1E-4.

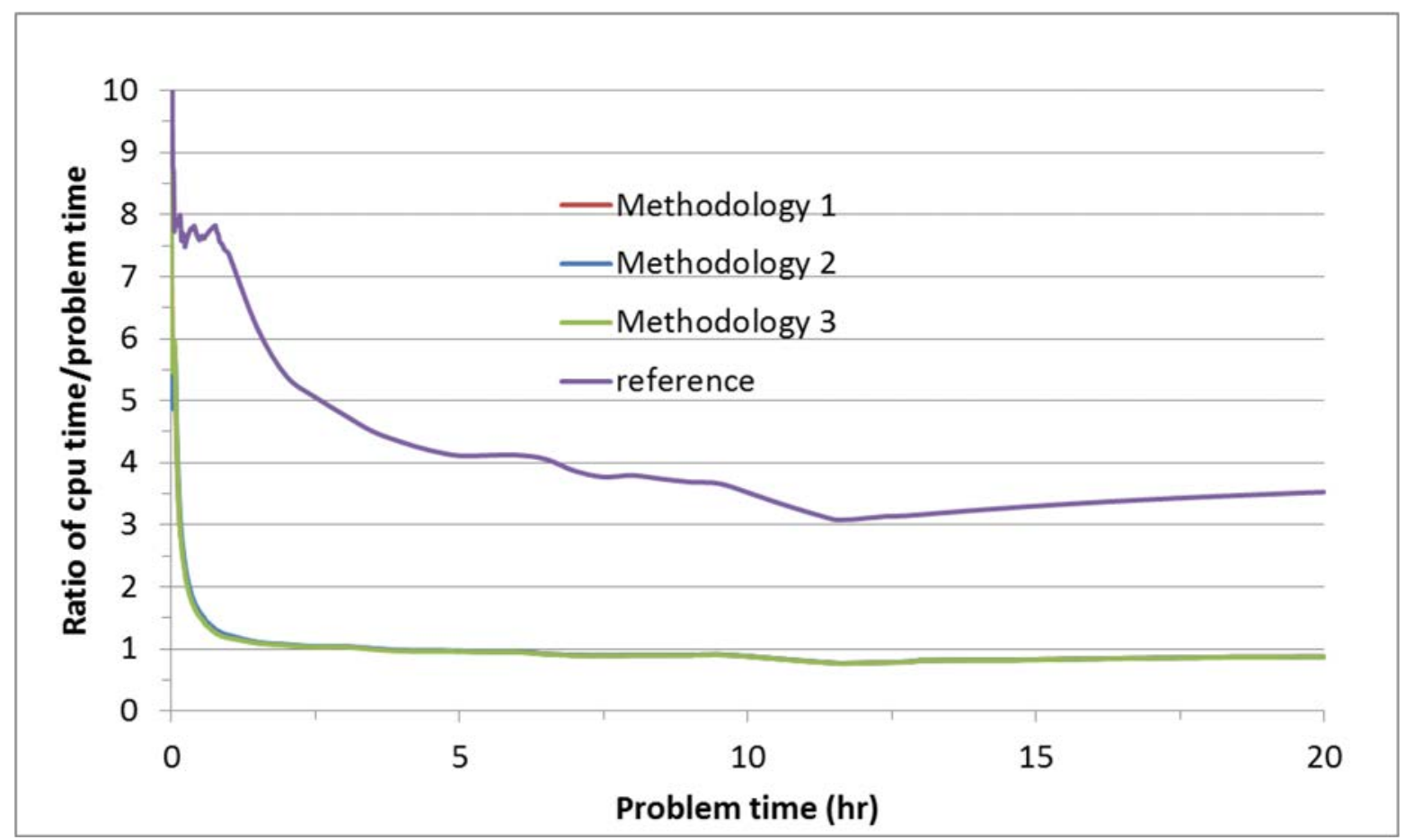

Figure 21. Time enhancement factor using a ratio of the cpu time to the problem time plotted over time for Methods 1, 2, and 3 using flux1_toll with a tolerance of 1E-4. 


\subsubsection{Method 1 Using Flux1_toll and Varying the Tolerance}

Similar to Section 4.1.2, tolerances used for Method 1 using flux1_toll were varied by a factor of ten greater than and less than the value of 1E-4 used in Section 4.1.3. Figure 22 through Figure 26 show data similar to those reported in previous sections. The highest tolerance value of $1 \mathrm{E}-5$ resulted in the lowest percent difference, similar to testing using flux_toll. Additionally, the cpu time used for the run with the highest tolerance (Table 6) completed almost 1.5 hours sooner than the other two methods with lower tolerance levels. Using a tolerance of $1 \mathrm{E}-5$ resulted in a time savings of $77.5 \%$. The shorter completion time for the higher tolerance was not an expected result and further review will be done to validate the obtained data.

Table 6. Run time summary for Method 1 using flux1_toll with tolerances of 1E-3, 1E-4, and 1E-5.

Test case

\begin{tabular}{ll} 
Reference & 70.87 \\
Method 1 tolerance $=1 \mathrm{E}-3$ & 17.30 \\
Method 1 tolerance $=1 \mathrm{E}-4$ & 17.55 \\
Method 1 tolerance $=1 \mathrm{E}-5$ & 15.98 \\
\hline
\end{tabular}
a 20 hour LOFC transient
Total CPU time (hours) to simulate

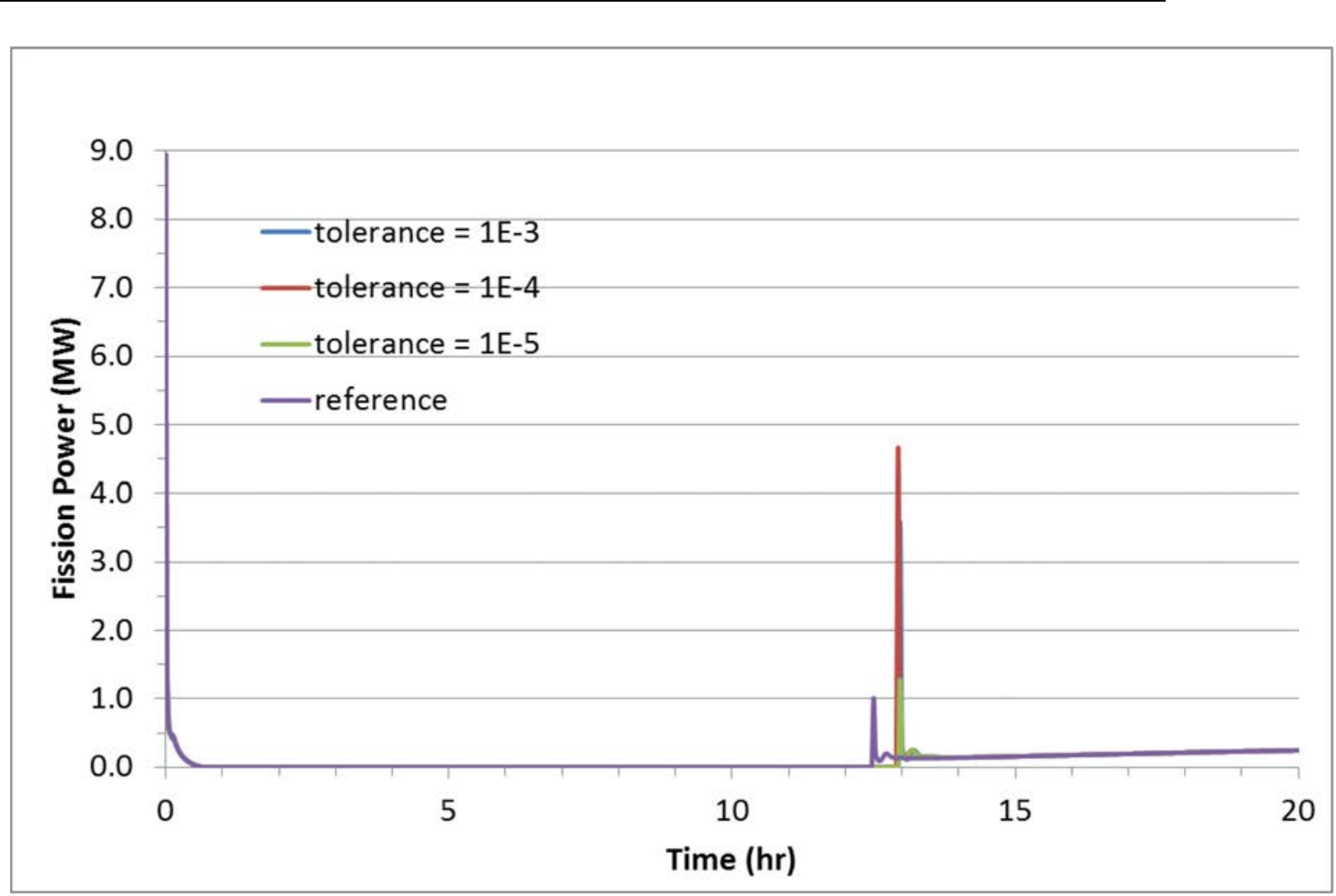

Figure 22. Fission power for the reference case and Method 1 flux1_toll using three different tolerance values: $1 \mathrm{E}-3,1 \mathrm{E}-4$, and $1 \mathrm{E}-5$. 


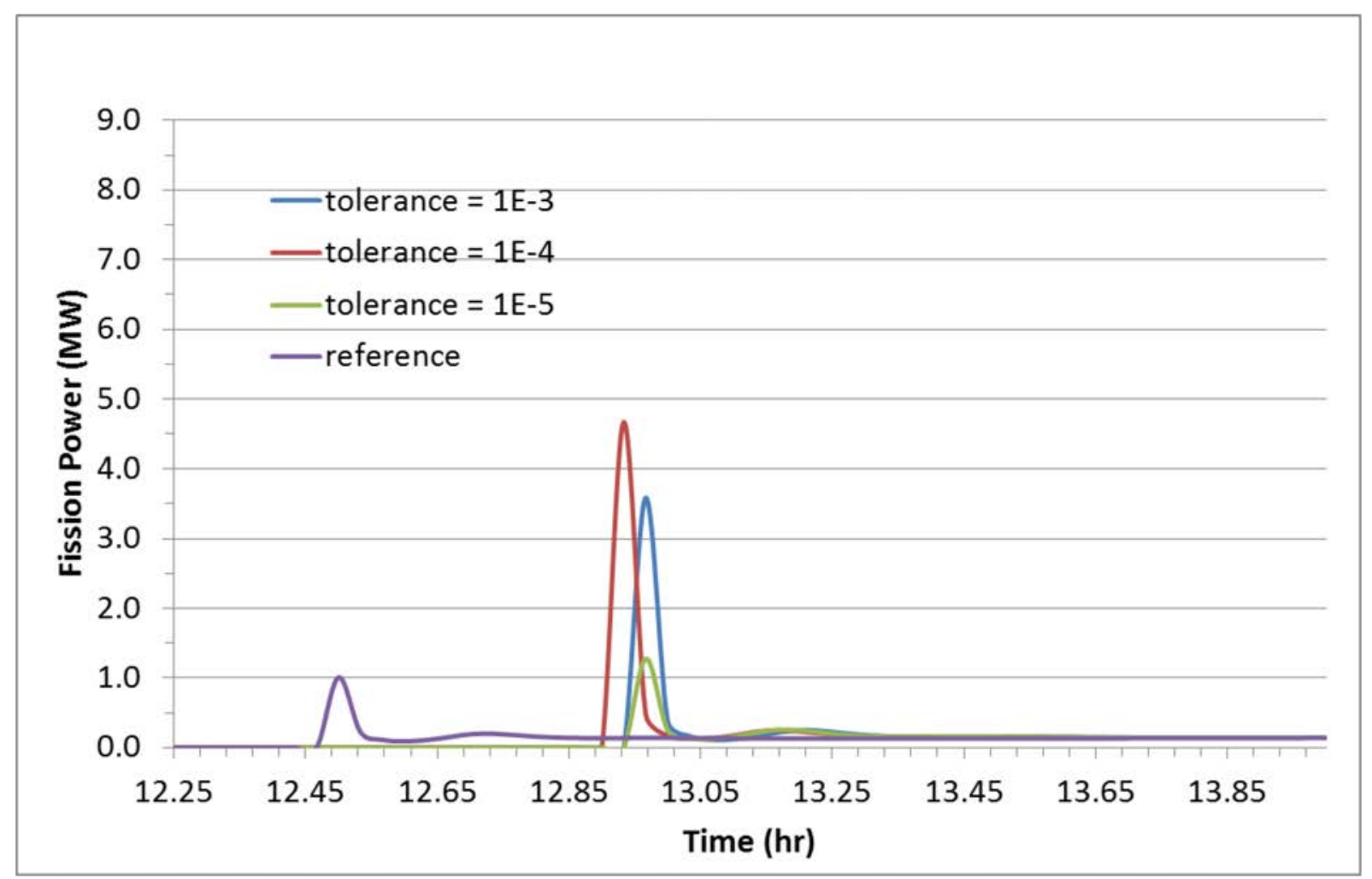

Figure 23. Fission power during the re-criticality period for the reference case and Method 1 flux1_toll using three different tolerance values: $1 \mathrm{E}-3,1 \mathrm{E}-4$, and $1 \mathrm{E}-5$.

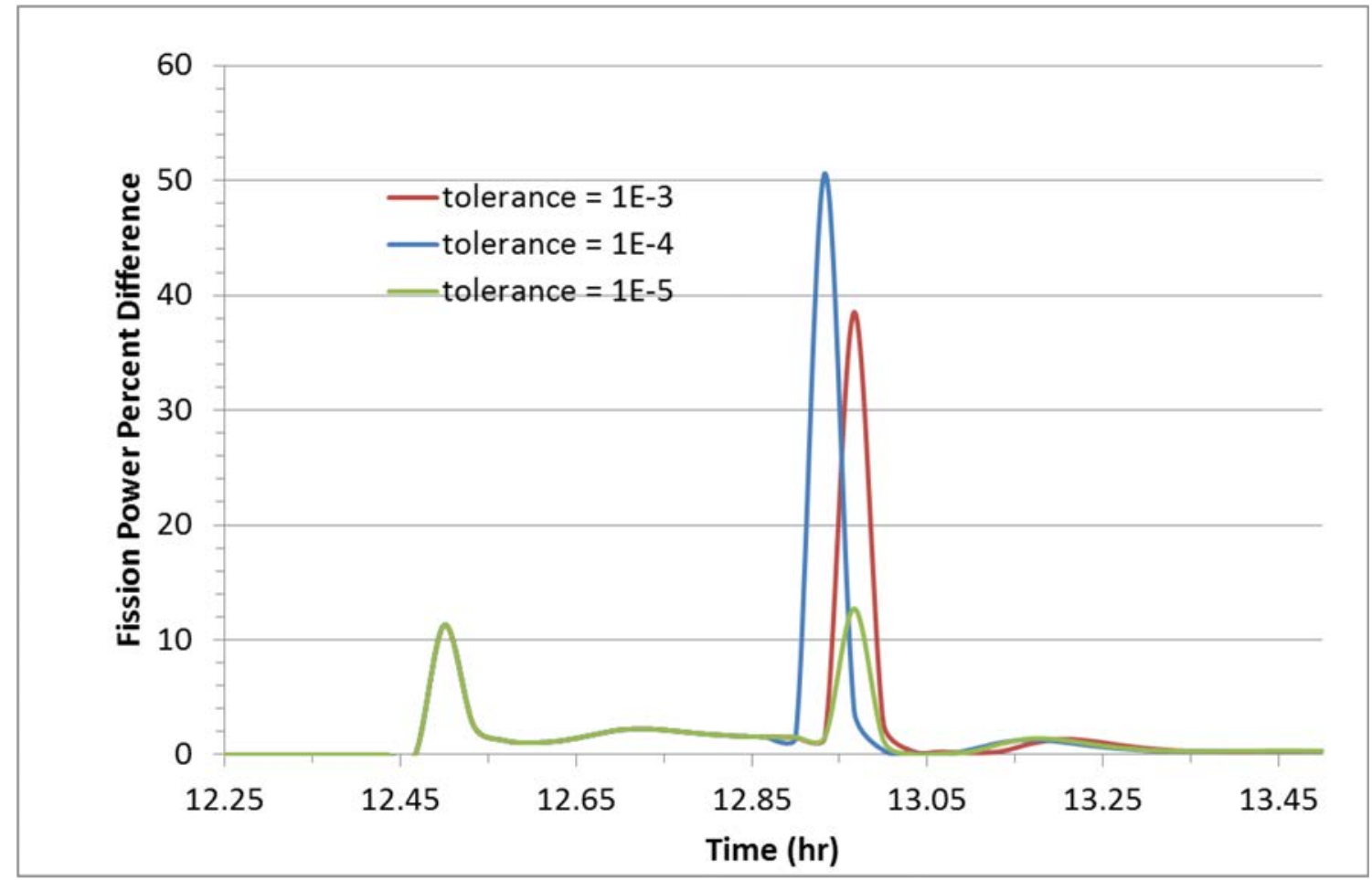

Figure 24. Fission power percent difference (from the reference case) for Method 1 using flux 1 _toll and three different tolerance values: $1 \mathrm{E}-3,1 \mathrm{E}-4$, and $1 \mathrm{E}-5$. 


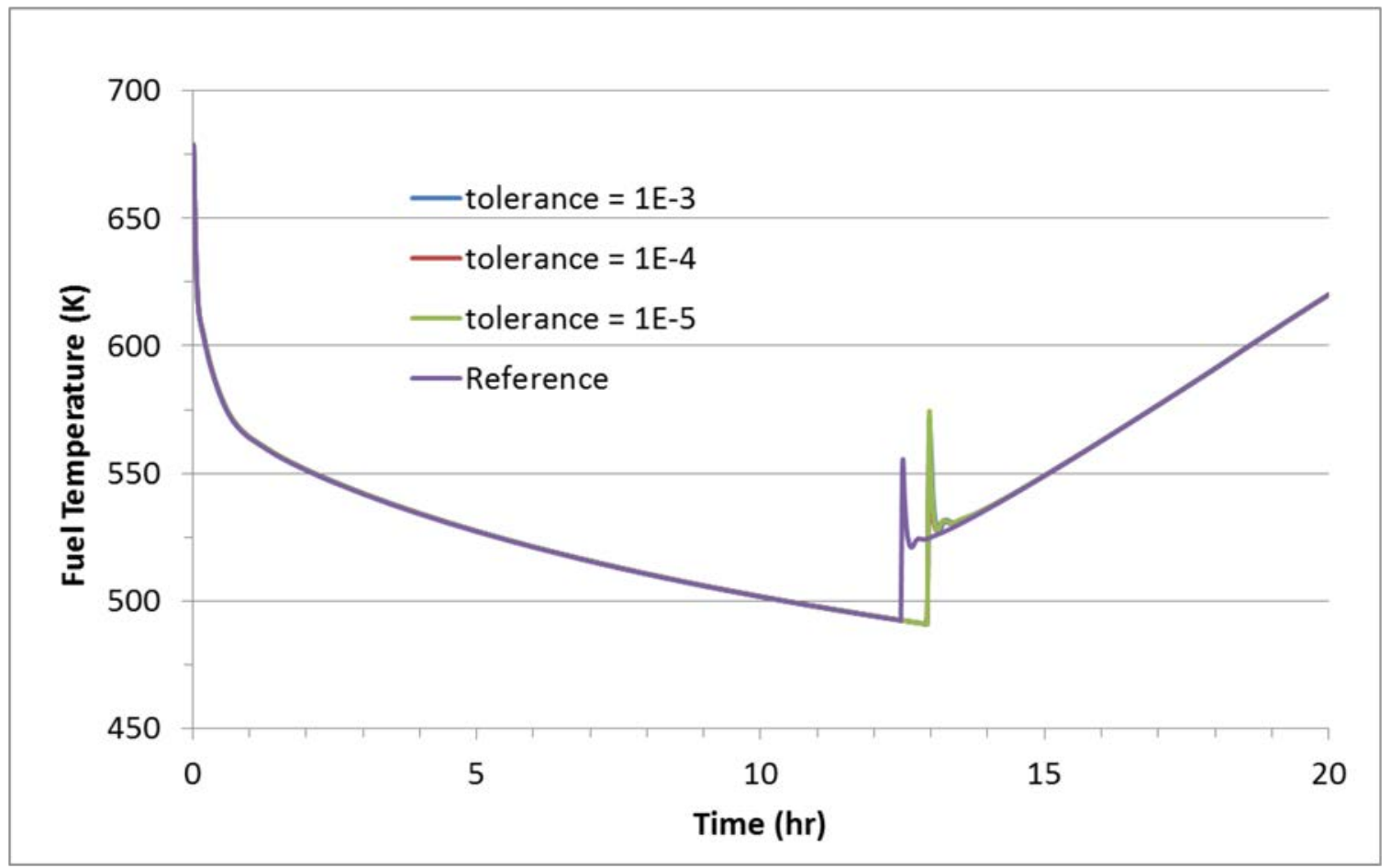

Figure 25. Ring 1 fuel block average temperature $(\mathrm{K})$ at the approximate axial core mid-point for the reference case and Method 1 flux1_toll using three different tolerance values: $1 \mathrm{E}-3,1 \mathrm{E}-4$, and $1 \mathrm{E}-5$.

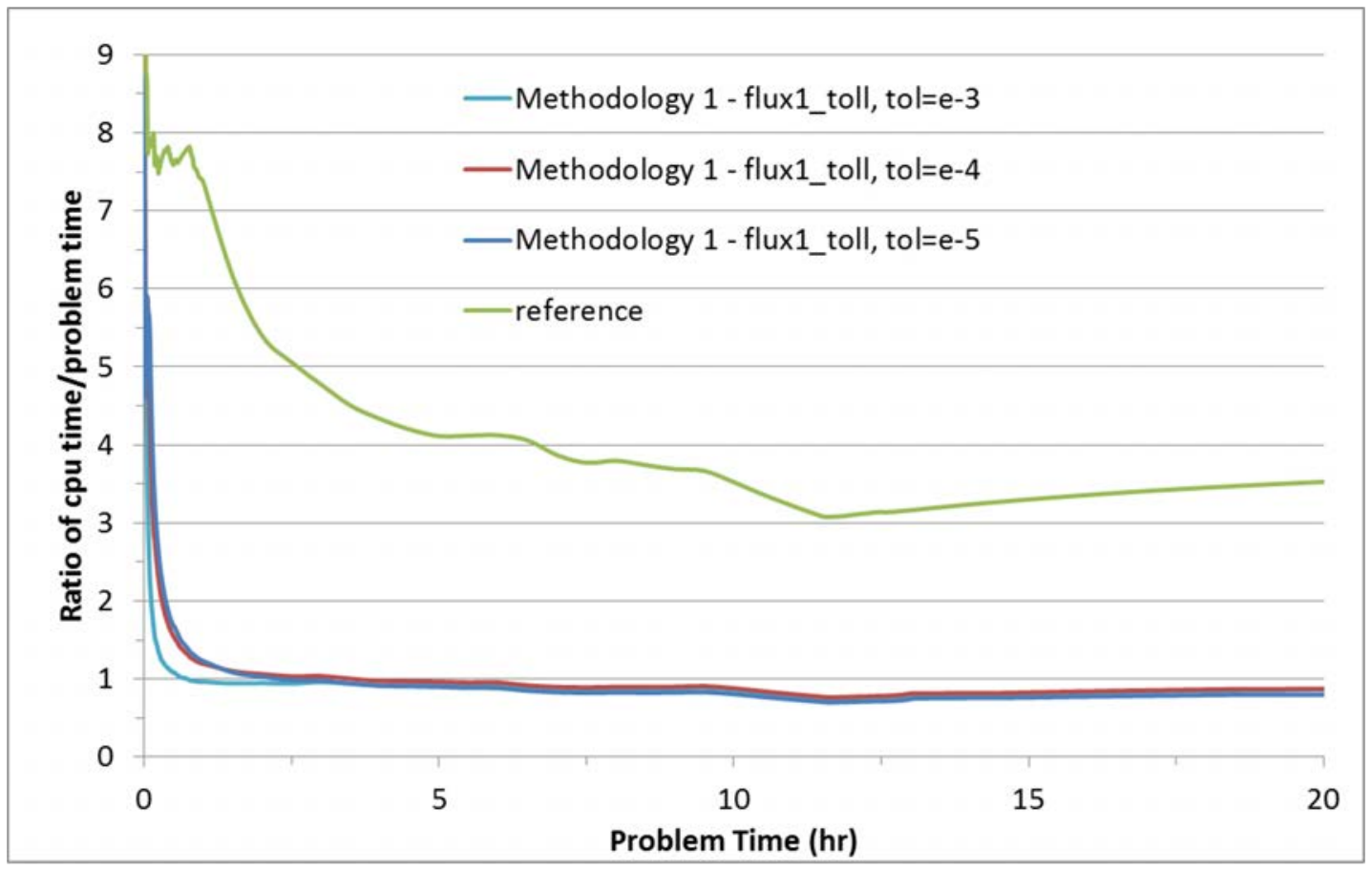

Figure 26. Time enhancement for the reference case and Method 1 using flux1_toll and three different tolerance values: $1 \mathrm{E}-3,1 \mathrm{E}-4$, and $1 \mathrm{E}--5$. 


\subsubsection{Method 2 using a Maximum Time Step Between Flux_toll and Flux1_toll}

In the final section of the 8-group calculations, a solver option is chosen that combines "the best of" the previous flux_toll and flux1_toll solvers. This option aims to optimize performance by using the largest time step allowed of the values calculated by the flux_toll and flux1_toll options.

To limit the number of calculations, only one method (\#2) and a tolerance setting have been chosen for this test, based on the recommendations from the first tests that were performed by the developer (Appendix B). The smaller model used in Appendix B did not lead to a re-critical phase, which lead to a recommendation to use Method 2 due to the large speed-up time factor that could be attained with reasonable accuracy. However, for the much larger HTTR model used here, the same performance using Method 2 in section 4.1.4 was not observed; although it achieved a speed-up factor on par with Method 3, it severely over-estimated the fission power peak and is not recommended for use. Therefore, Method 3 would have been the preferred option to test in this section, but due to time and HPC resource limitations, only the performance of the combined solver using Method 2 was included here. It is recommended that an additional test using Method 3 should be performed to confirm the findings of this section.

Figure 27 through Figure 29 provide the results of these tests. The re-critical power peak magnitude is very close to the reference; however, it is delayed by about 30 minutes. The percent difference during the initial power reduction is high due to offset of the timing; the difference during the re-criticality is mostly due to the delay time of the re-criticality. The time savings observed in Figure 29 is very good; most of the accelerated calculation was performed three to four times faster than the reference calculation. The total simulation time of 17.6 hours is about four times faster than the 70.9 hours for the reference run (Table 7), but it is not significantly faster than just using the flux1_toll option.

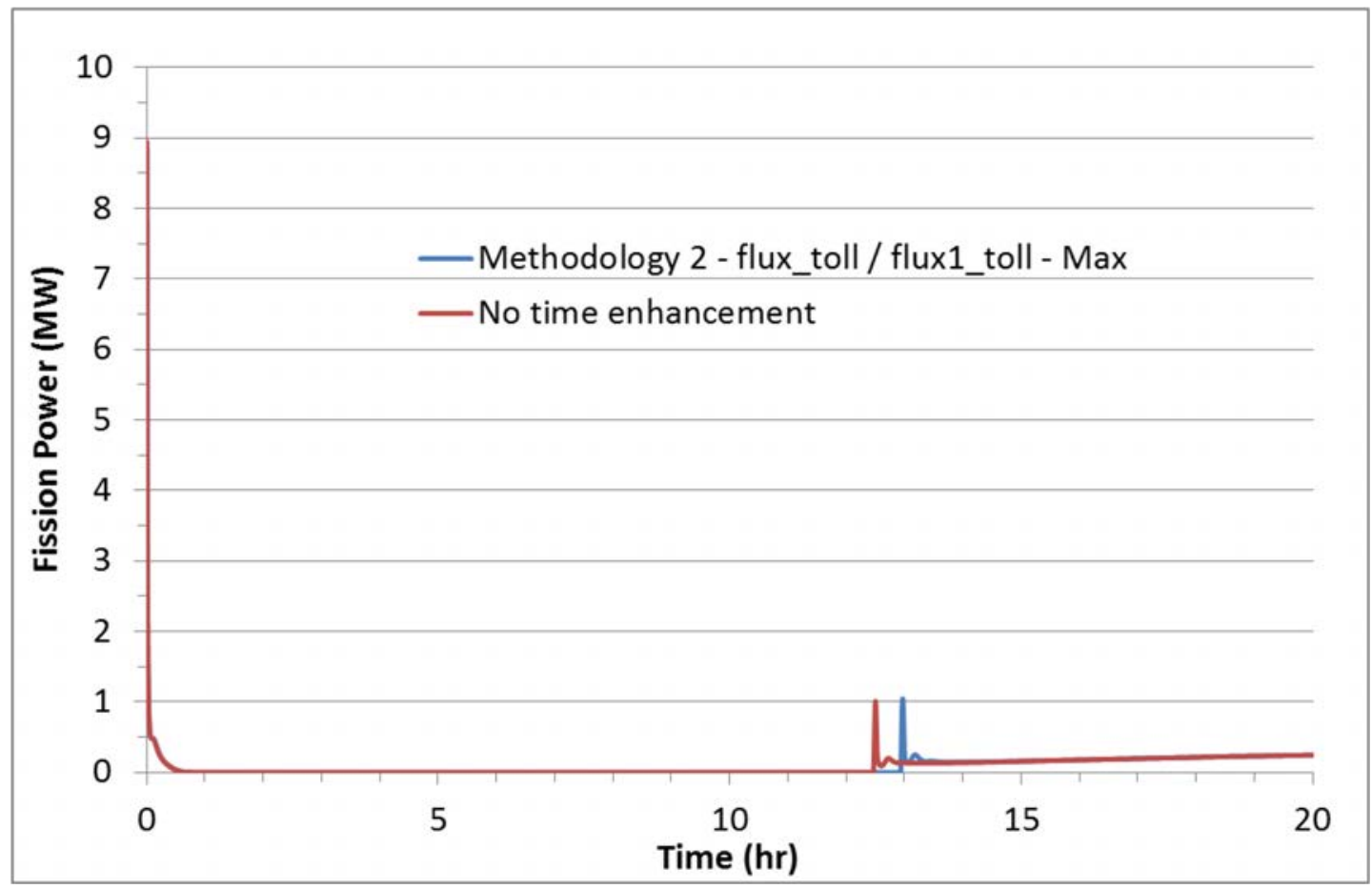

Figure 27. Fission power for the reference case and Method 2 using maximum time step between flux_toll and flux1_toll with tolerances of $1 \mathrm{E}-5$ and $1 \mathrm{E}-4$, respectively. 


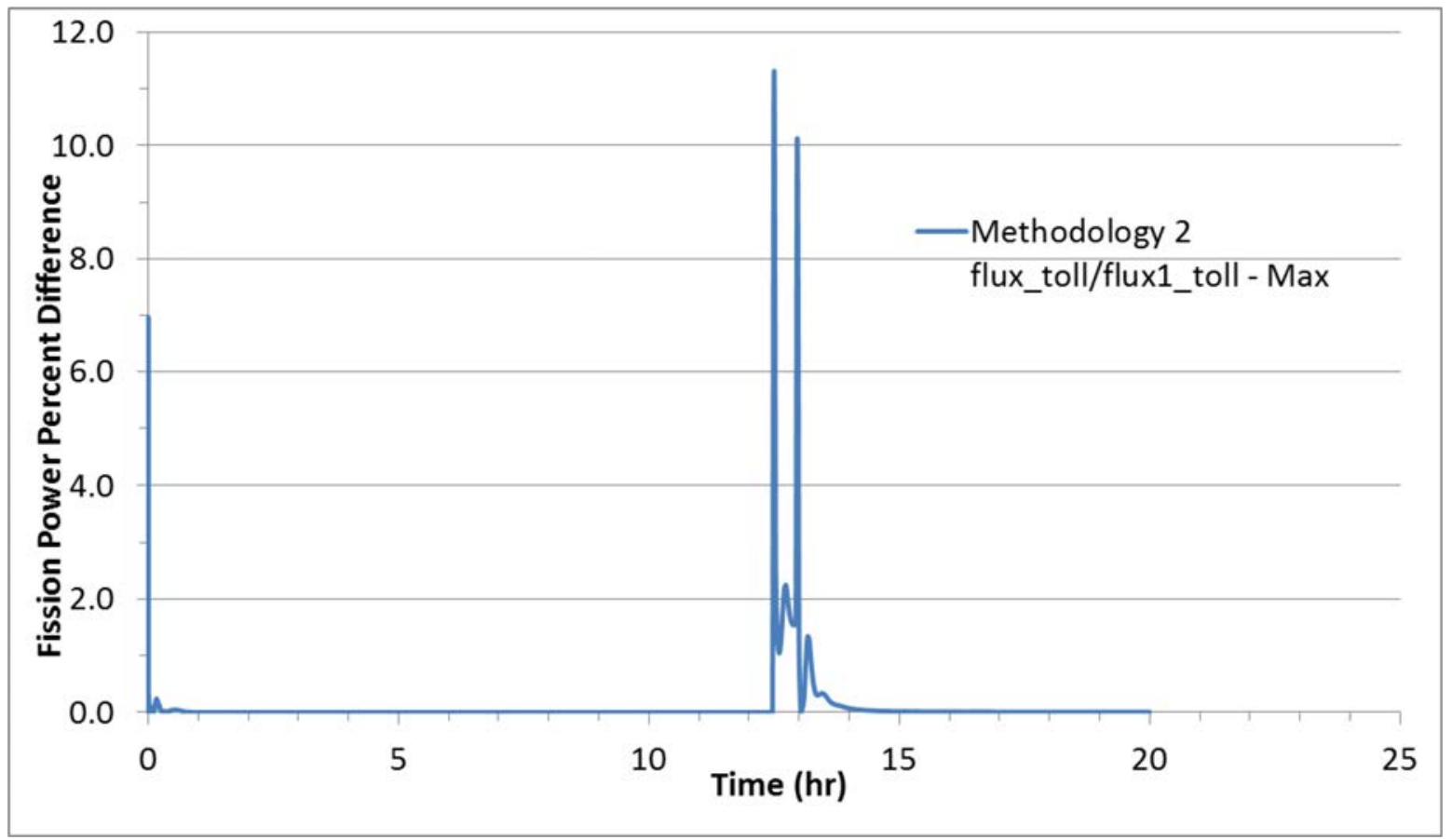

Figure 28. Fission power percent difference (from the reference case) for Method 2 using the maximum time step between flux_toll and flux1_toll with tolerances of $1 \mathrm{E}-5$ and $1 \mathrm{E}-4$, respectively.

Table 7. Run time summary for Method 2 using maximum time step between flux_toll (tolerance=1E-5) and flux1_toll (tolerance=1E-4).

\begin{tabular}{lc}
\hline \multicolumn{1}{c}{ Test Case } & $\begin{array}{c}\text { Total CPU Time (Hours) to Simulate } \\
\text { an 20 Hour LOFC Transient }\end{array}$ \\
\hline Reference & 70.9 \\
Method 2 with Maximum time step & 17.6 \\
between flux_toll and flux1_toll & \\
\hline
\end{tabular}




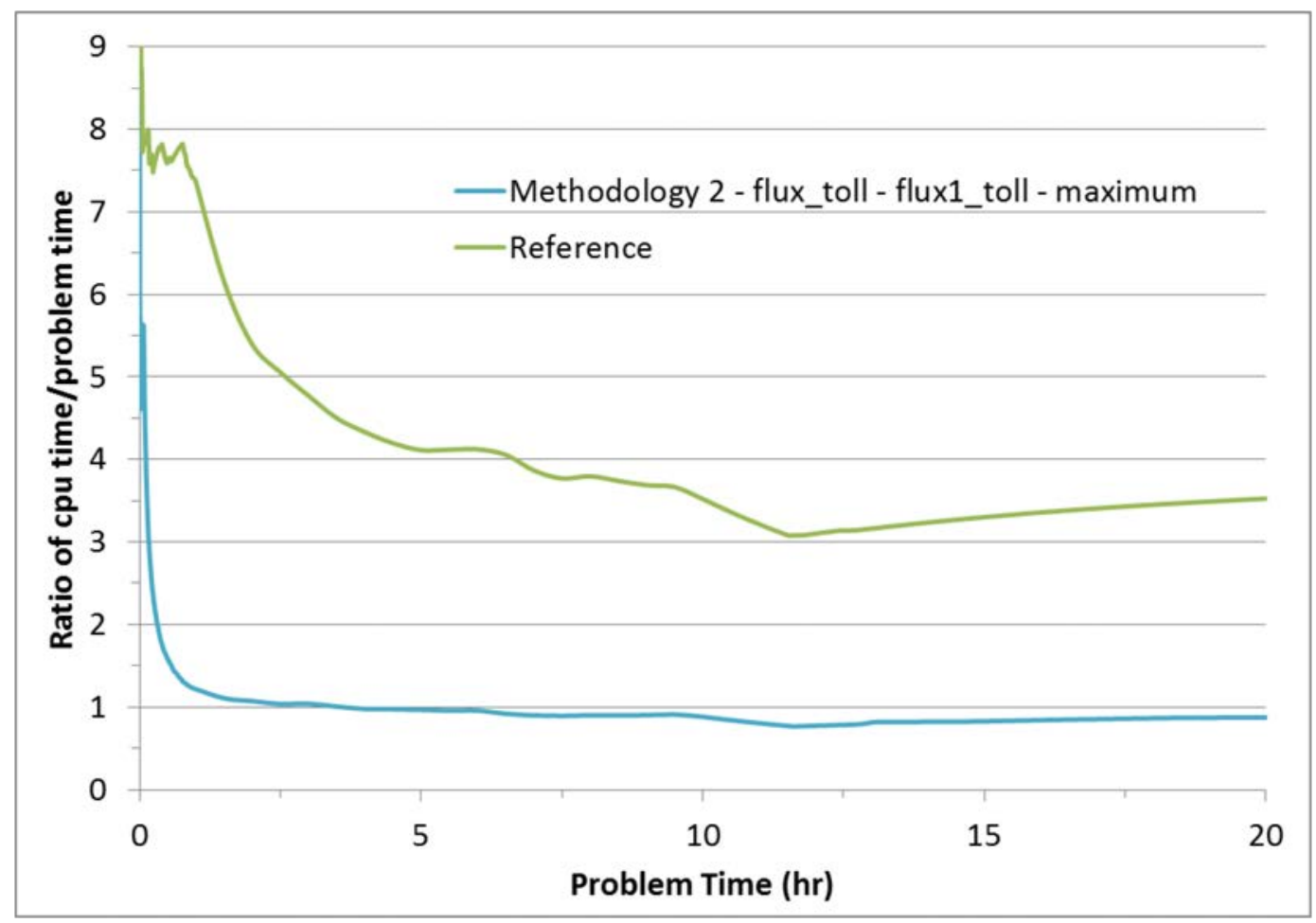

Figure 29. Time enhancement for the reference case and Method 2 using the maximum time step calculation between flux_toll and flux1_toll with tolerances of $1 \mathrm{E}-5$ and $1 \mathrm{E}-4$, respectively.

\subsubsection{8-group Model Summary}

In conclusion to Section 4.1, the main findings are as follows:

- The choice of both the method and tolerance settings are very important to the level of time enhancement achieved and the accuracy of the results, compared with a calculation that does not use any adaptive time stepping.

- Use of Method 1 flux_toll option with a tolerance of 1E-5 did not result in a significant improvement in performance.

- Relaxing the tolerance setting to 1E-4 for Method 1 flux_toll improved the speed-up factor significantly (to almost real-time performance), with an acceptable accuracy level for scoping calculations. If higher fidelity results are desired, a tolerance setting of $1 \mathrm{E}-5$ is recommended.

- The flux1_toll option performed much better than the flux-toll option in terms of the speed-up achieved. No significant differences were seen when the "best-of" approach for using both types was applied; therefore, it seems that the use of just the flux1_toll option is sufficient.

- From the results presented in this section, it is recommended that the best-performing option in terms of time-enhancement and accuracy should be Method 3 using flux1_toll and a tolerance of 1E-4. An even lower setting of 1E-3 should also be tested, especially if the intended use is scoping studies. A significant speed-up improvement of the cpu/problem time factor should be achieved, ranging from a factor of three to eight times faster than the reference calculation. An 8-group HTTR LOFC calculation running close to or at real-time should be possible.

- Method 2 using flux1_toll with a tolerance of 1E-5 is not recommend for the HTTR model, because it leads to unacceptable predictions of the re-critical fission power peak. 


\subsection{Results Using 26 Neutron Energy Groups}

\subsubsection{Methods 1, 2, and 3 Using Flux_toll and a Tolerance of 1E-5}

The distribution of time spent by the neutronics and thermal hydraulics solvers during the PHISICS/RELAP5-3D time-dependent iteration scheme depends on many factors and is a varying function over time (see Section 4.1). Because the neutronics module takes significantly longer to solve the transport equation for 26-groups than for 8-groups, it can be expected that the performance of the adaptive time-step methods would be better (or more efficient) for the 26-group cases, because the adaptive time-step improvements only operate on the neutronics module. As stated before, the RELAP53D thermal hydraulic model coupled to the 8-group PHISICS model is identical to that coupled to the26group PHISICS model, so in addition to some overhead, the 26 group simulation should be at least three times slower than the 8-group simulation.

It is shown in Table 8 that the reference 26-group calculation (i.e., without time enhancement) took a total of about 210 hours to complete 18 hours of simulation time, compared with 62 hours for the 8-group simulation for 18 hours of simulation time (i.e., a factor of about 3.4 times slower). (The 26-group simulations were terminated after 8.75 days on the HPC before they could be fully completed to 20 hours). All three methods finished in approximately the same time (Table 8) and, consistent with the 8group results, the flux_toll option does not provide any time enhancement for the run (Figure 30).

Figure 31 shows that the timing of the re-critical fission power peak is similarly predicted by all three methods, and the magnitudes are almost identical to the reference value (approximately $1 \mathrm{MW}$ ). The fuel block temperature data (Figure 32) are consistent with the power behavior.

Based on testing results using flux_toll with a tolerance of $1 \mathrm{E}-5$, the conclusion is that these options do not provide significant time enhancements.

Table 8. Run time summary for Methods 1,2 and 3 using flux_toll with a tolerance of 1E-5.

\begin{tabular}{lcc} 
& Test Case & $\begin{array}{c}\text { Total CPU Time (Hours) to Simulate } \\
\text { an 18 Hour LOFC Transient }\end{array}$ \\
\hline Reference & 211.4 \\
Method 1 & 197.6 \\
Method 2 & 204.4 \\
Method 3 & 197.6 \\
\hline
\end{tabular}




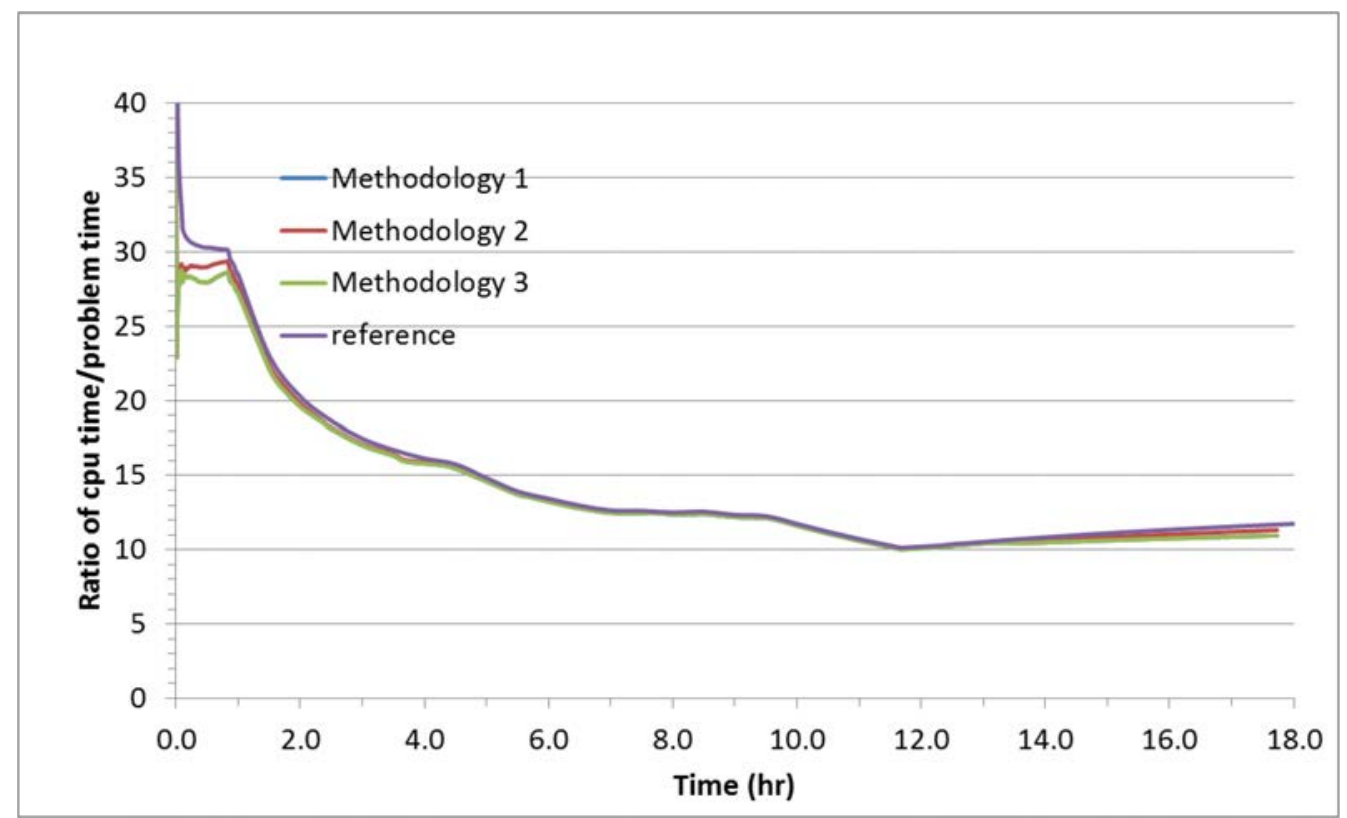

Figure 30. Time enhancement factor using a ratio of the cpu time to the problem time plotted over time for Methods 1, 2, and 3 using flux toll with a tolerance of $1 \mathrm{E}-5$.

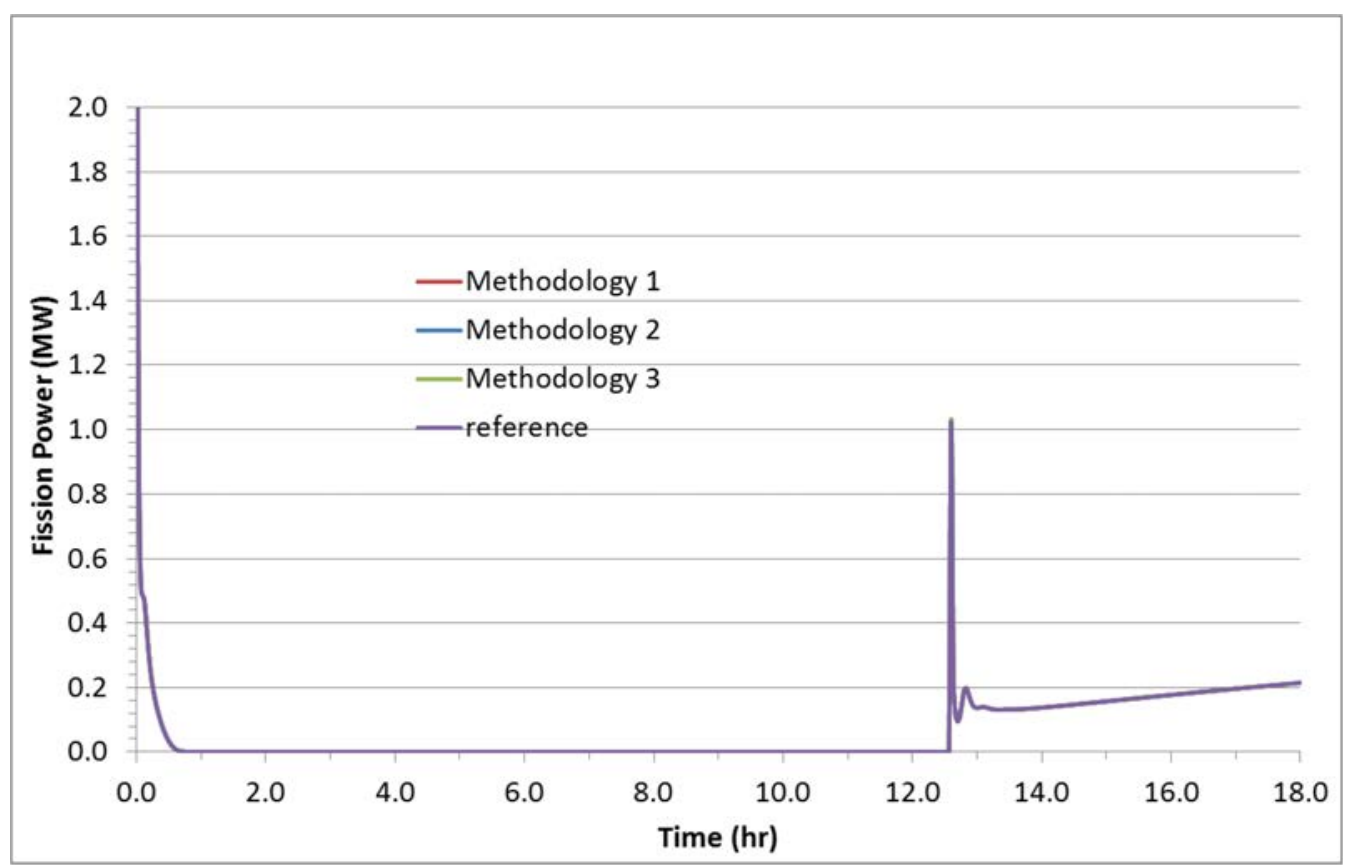

Figure 31. Fission power (MW) for the reference case and Methods 1, 2, and 3 using flux_toll with a tolerance of $1 \mathrm{E}-5$. 


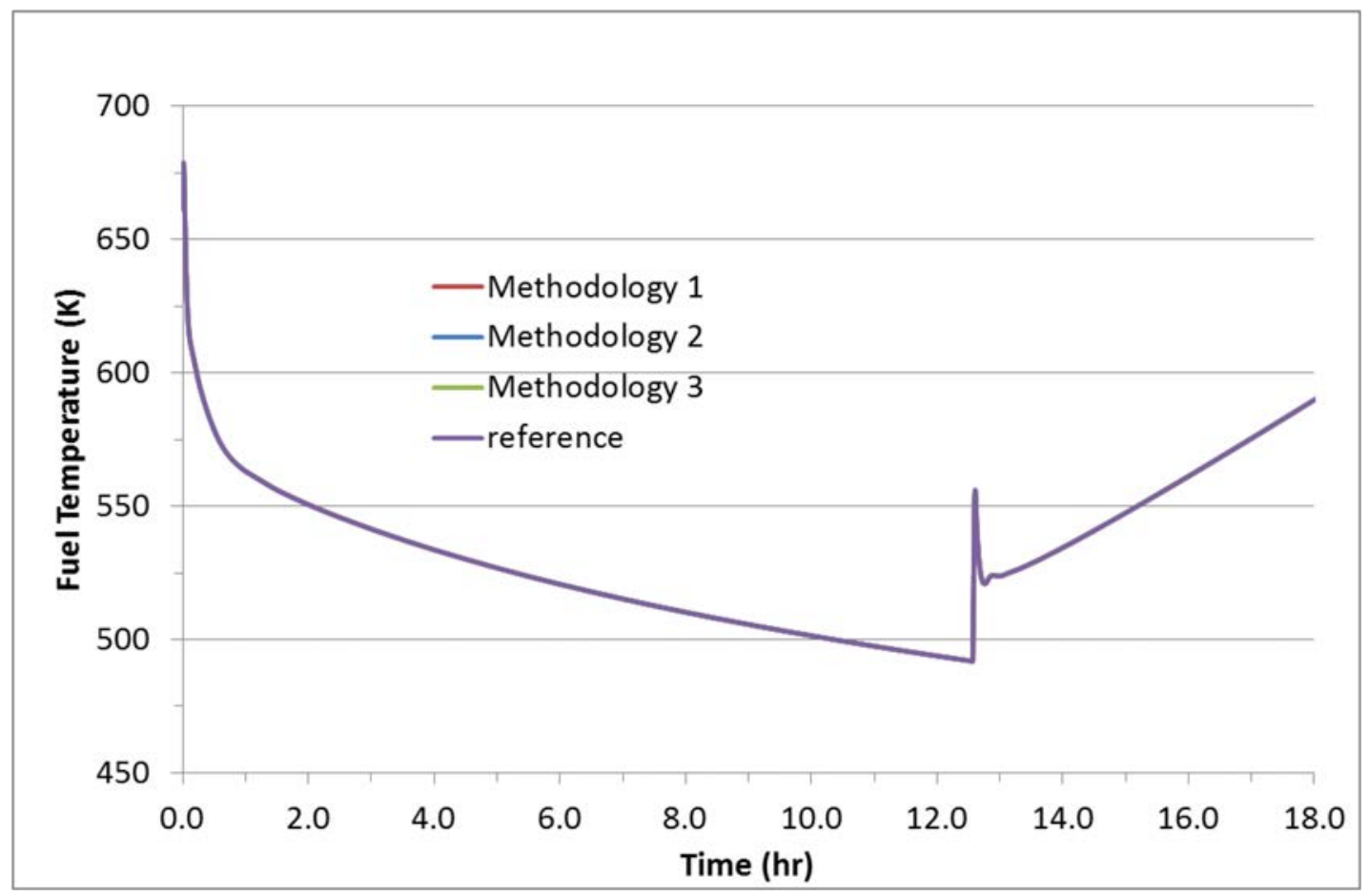

Figure 32. Ring 1 fuel block average temperature $(\mathrm{K})$ at the approximate axial mid-point for the reference case and Methods 1, 2, and 3 using flux_toll with a tolerance of $1 \mathrm{E}-5$.

\subsubsection{Method 1 Using Flux_toll and Varying the Tolerance}

It was concluded in Section 4.1.2 that use of a 1E-4 tolerance setting could be quite useful for scoping studies when absolute accuracy is not important, but rapid model development and debugging is required. A similar conclusion can also be reached in the case of the 26-group simulation as well. Figure 33 shows that fission power behavior was not affected by an increase or decrease of the tolerance setting by an order of magnitude (i.e., all three curves overlap). Improvement in simulation time is likewise noticeable for the 1E-4 setting (Figure 34), with this simulation finishing in 150 hours compared to the approximate 210 hours for the reference run (Table 9). Based on these results, it is recommended that a tolerance of $1 \mathrm{E}-4$ be utilized for Method 1 using flux_toll.

Table 9. Run time summary for Method 1 using flux_toll with tolerance values of 1E-4, $1 \mathrm{E}-5$, and $1 \mathrm{E}-6$.

Test Case

\section{Reference}

Method 1 tolerance $=1 \mathrm{E}-4$

Method 1 tolerance $=1 \mathrm{E}-5$

Method 1 tolerance $=1 \mathrm{E}-6$
Total CPU Time (Hours) to Simulate a 18 Hour LOFC Transient

211.4

150.4

197.6

209.3 


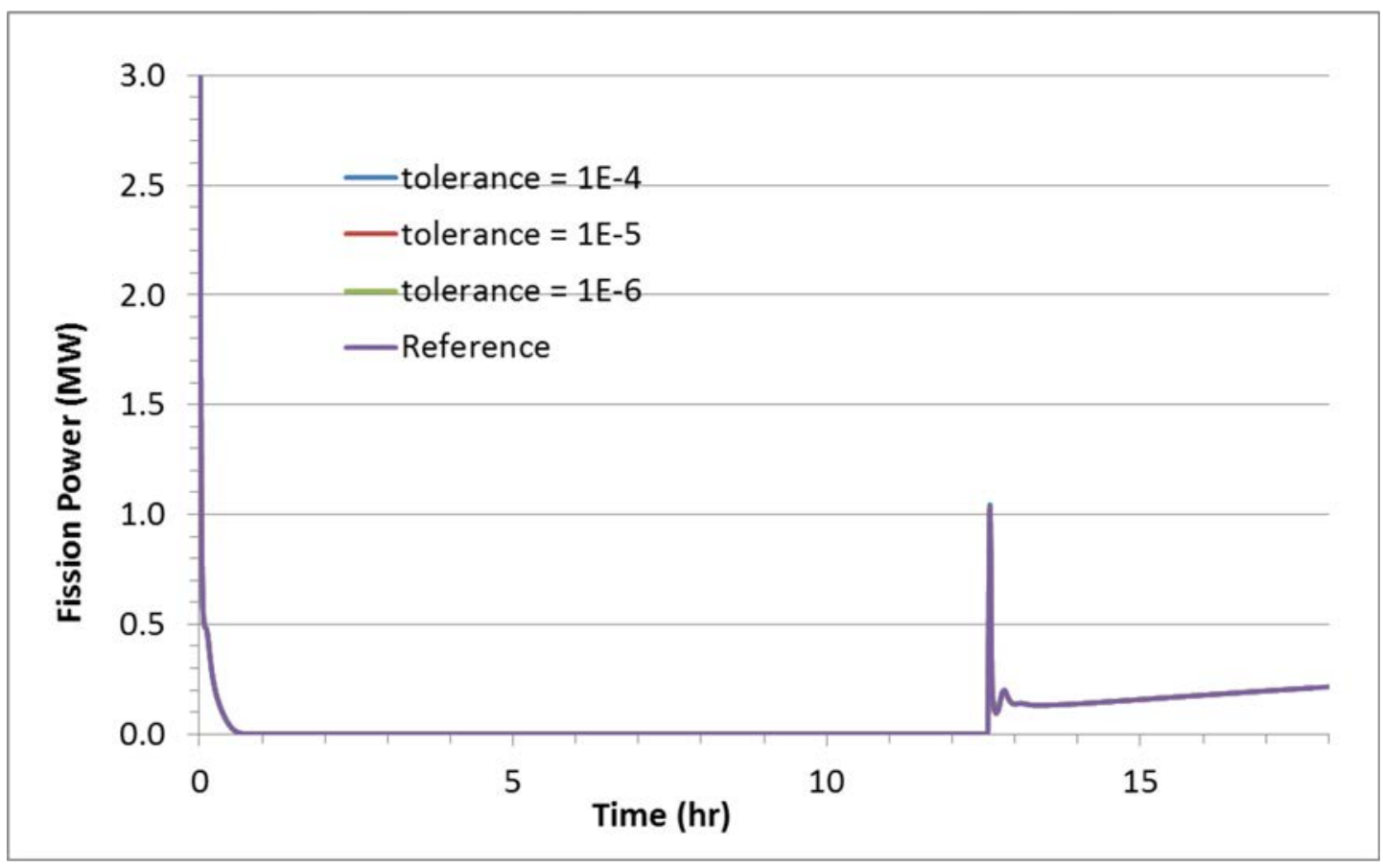

Figure 33. Fission power (MW) for the reference case and Method 1 flux_toll using three different tolerance values: $1 \mathrm{E}-4,1 \mathrm{E}-5$, and $1 \mathrm{E}-6$.

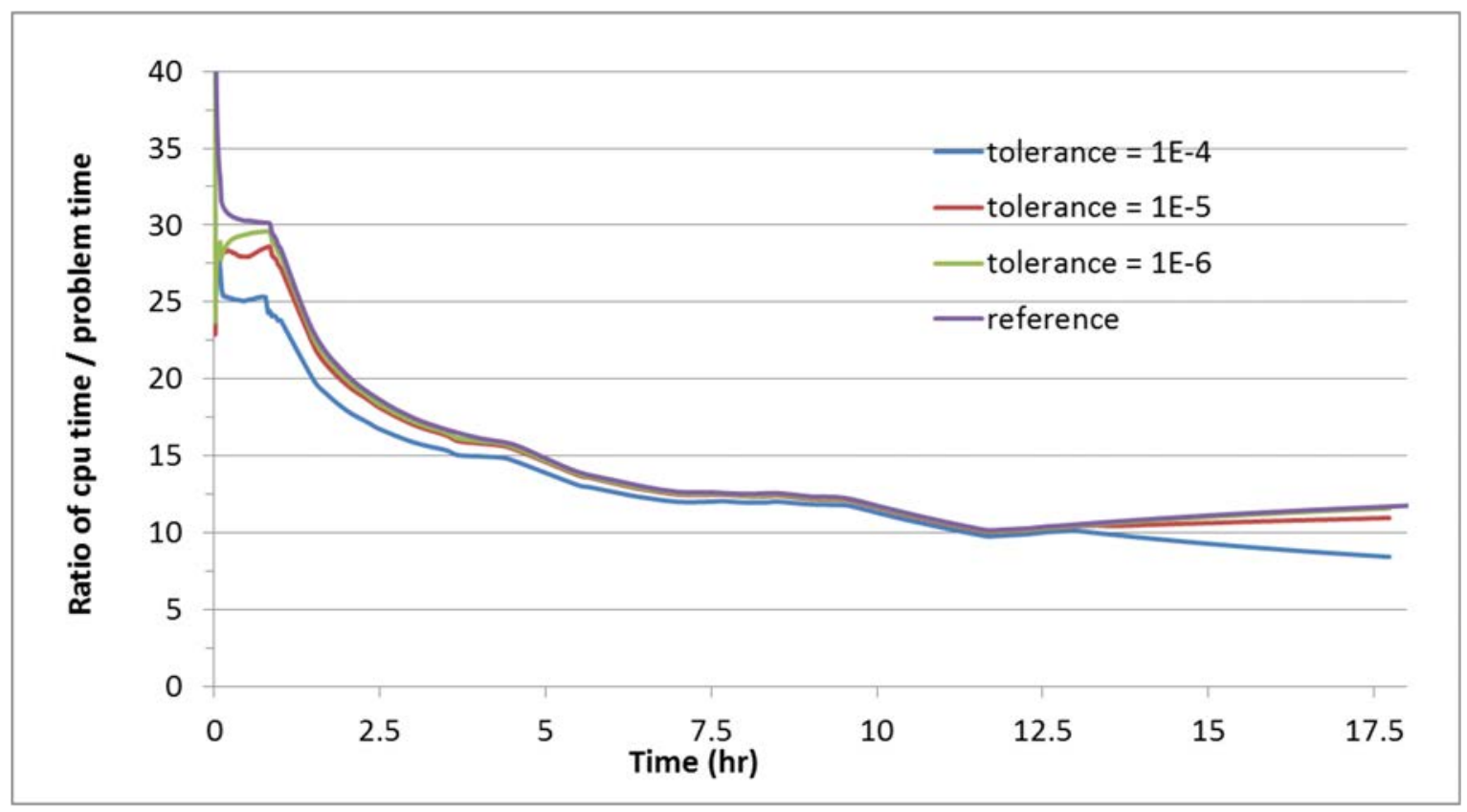

Figure 34. Time enhancement for the reference case and Method 1 using flux_toll and three different tolerance values: $1 \mathrm{E}-4,1 \mathrm{E}-5$, and $1 \mathrm{E}-6$.

\subsubsection{Methods 1, 2, and 3 Using Flux1_toll}

In this section, the same comparisons are performed as in Section 4.1.3 for the flux1_toll option, and a tolerance setting of 1E-4. A speed-up factor of 3.5 was achieved by using these settings for all three methods (Figure 35 and Table 10). 
The re-critical power and fuel temperature peaks calculated using the flux1_toll option for each of the methods is offset from the reference by approximately 30 minutes (Figure 36). These trends are similar to the results obtained for the 8-group simulations in Section 4.1.3, but interestingly not for the same methods. For the 8-group case, it was found that Method 2 resulted in the calculation of a re-critical power peak that is about 12 times greater than the reference calculation, where in the case of 26-group case it is Method 1 that leads to a very large difference (i.e., about a seven times larger fission peak; Figure 37). Method 3 actually seems to perfom the best for the 26-group simulation, based on the magnitude of the re-critical peak similarity to the reference, consistent with what was recommended for use in the 8-group simulation. It is not clear at this point why use of these Methods lead to different trends when used with more energy groups. This aspect should be investigated further in future iterations of this HTTR model.

The fuel temperature trend (Figure 38) shows a greater increase in the temperature for Method 1 (approximately $140 \mathrm{~K}$ ) as compared to the 8-group calculation for Method 2 (approximately $100 \mathrm{~K}$ ) and the 8-group calculation resulted in a greater magnitude for its re-critical peak.

Table 10. Run time summary for the 26-group simulation using Methods 1, 2 and 3 and flux1_toll with a tolerance of $1 \mathrm{E}-4$.

Test Case

Total CPU Time (Hours) to Simulate an 18 Hour LOFC Transient

\begin{tabular}{lr} 
Reference & 211.4 \\
Method 1 & 52.4 \\
Method 2 & 52.6 \\
Method 3 & 52.6 \\
\hline
\end{tabular}

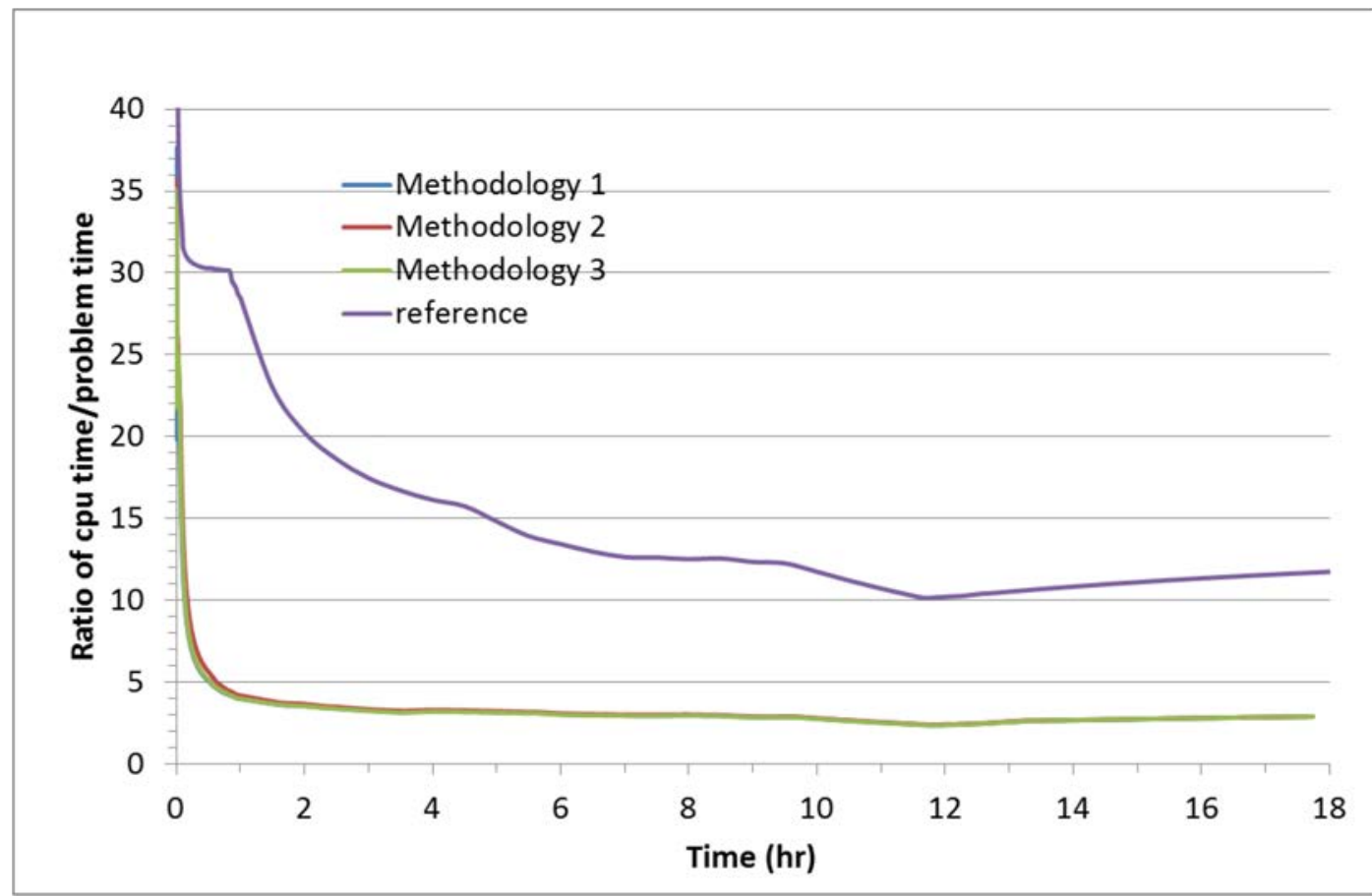

Figure 35. Time enhancement factor using a ratio of the cpu time to the problem time plotted over time for Methods 1, 2, and 3 using flux1_toll with a tolerance of 1E-4. 


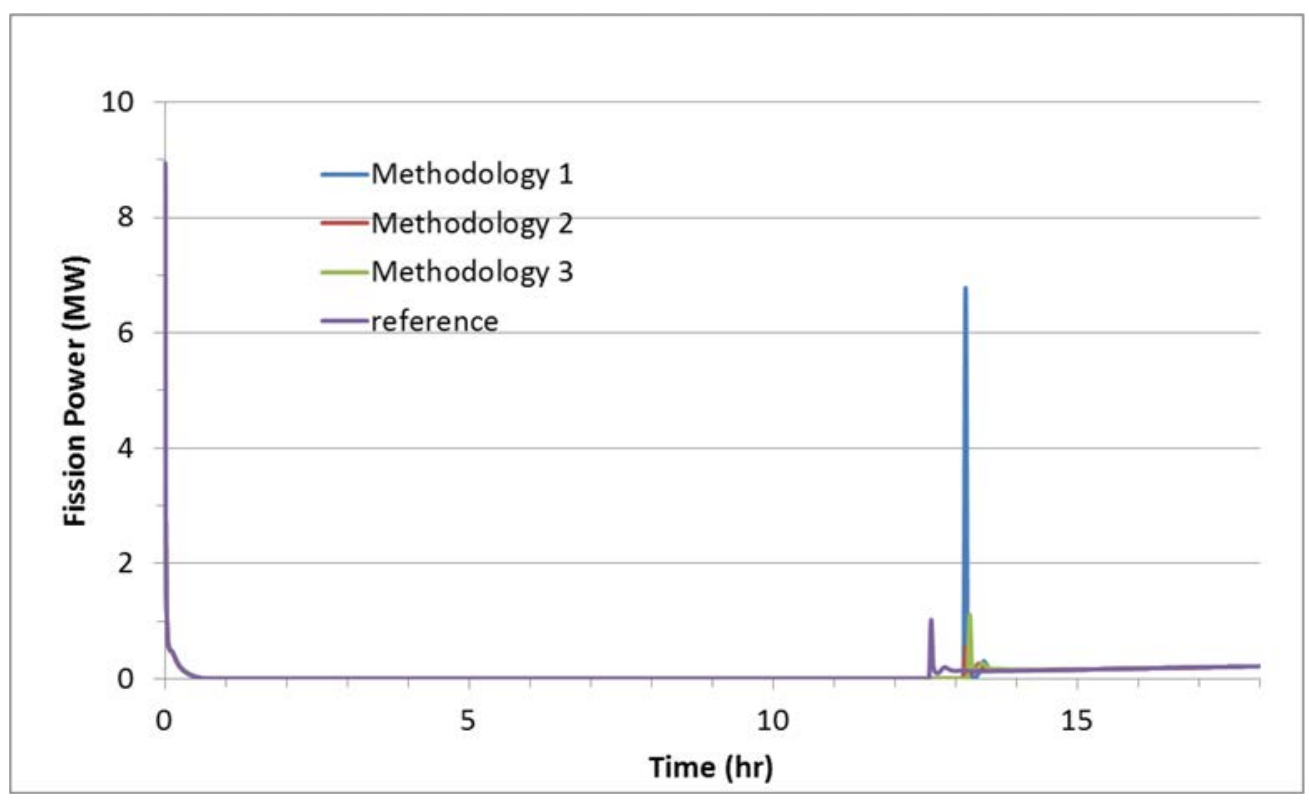

Figure 36. Fission power (MW) for the reference case and Methods 1, 2, and 3 using flux1_toll with a tolerance of $1 \mathrm{E}-4$.

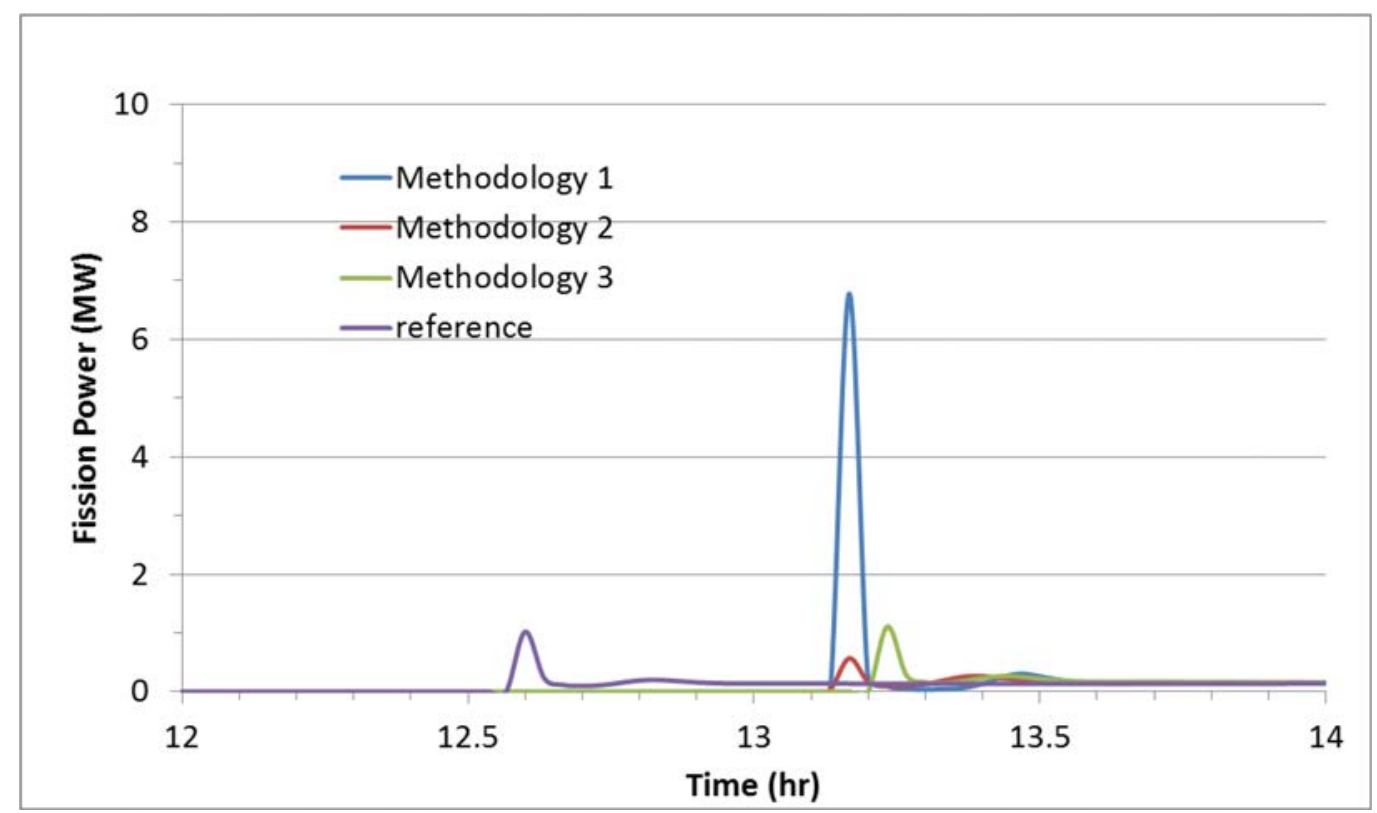

Figure 37. Fission power during the re-criticality portion of the transient for the reference case and Methods 1, 2, and 3 using flux1_toll with a tolerance of 1E-4. 


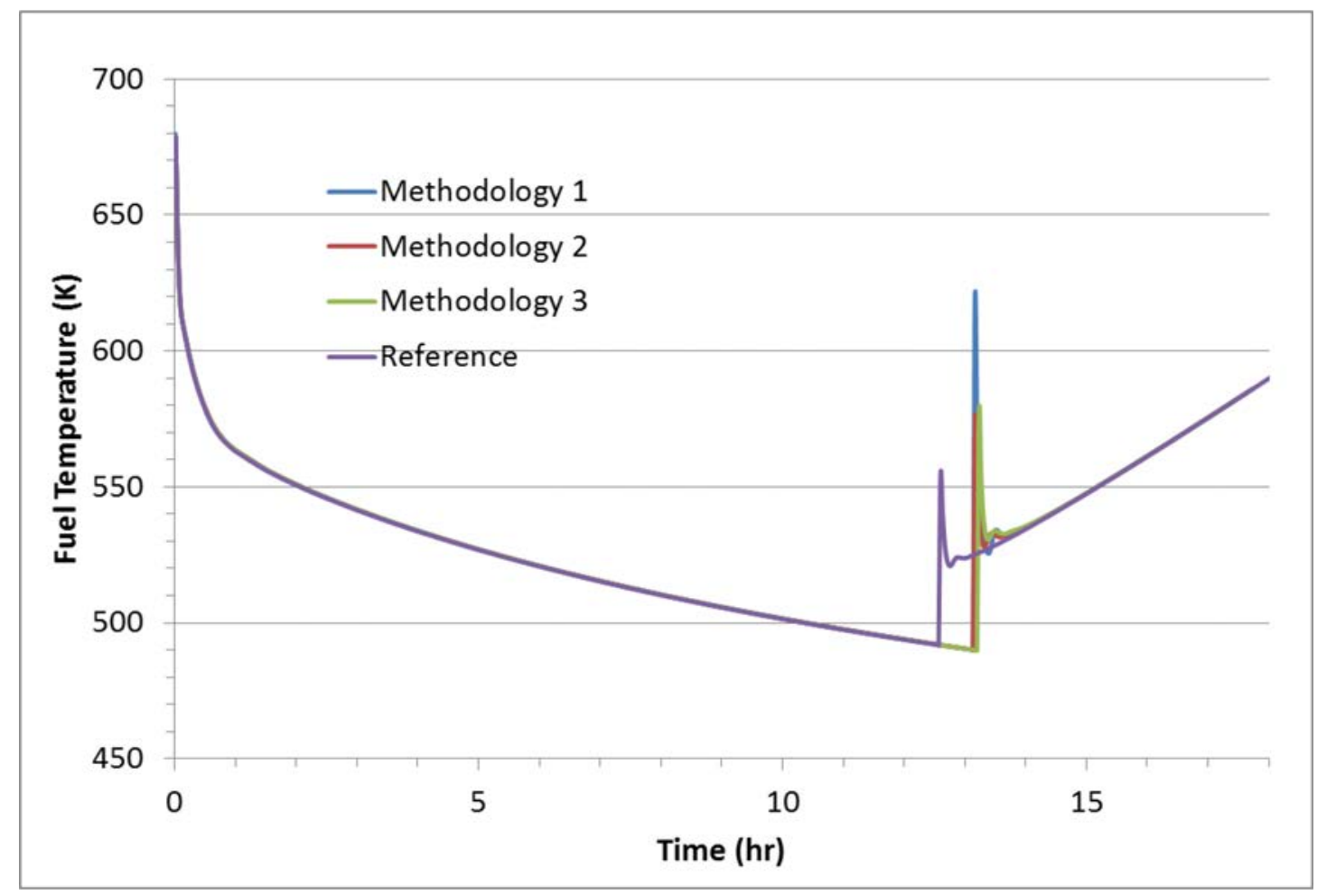

Figure 38. Ring 1 fuel block average temperature $(\mathrm{K})$ at the approximate axial mid-point for the reference case and Methods 1, 2, and 3 using flux1_toll with a tolerance of 1E-4.

\subsubsection{Method 1 Using Flux1_toll and Varying the Tolerance}

The results obtained with Method 1 using the flux1_toll option with the 26-group model (Figure 39 and Figure 40) indicate that a tolerance of 1E-4 is the worst performing due to the high re-critical peak and $1 \mathrm{E}-3$ and $1 \mathrm{E}-5$ are almost identical. The 8 -group results showed the $1 \mathrm{E}-3$ tolerance value to be the best option. The cpu times for each case (Table 11 and Figure 41) are consistent with an approximate factor of 4.0 .

Because Method 3 from Section 4.2.3 performed the best on this HTTR, testing using Method 3 and flux1_toll with a tolerance of $1 \mathrm{E}-3$ is recommended.

Table 11. Run time summary for Method 1 using flux1_toll with tolerances of 1E-3, 1E-4, and 1E-5.

\section{Test case}

Reference
Method 1 tolerance $=1 \mathrm{E}-3$
Method 1 tolerance $=1 \mathrm{E}-4$
Method 1 tolerance $=1 \mathrm{E}-5$

Total CPU time (hours) to simulate a 18 hour LOFC transient 


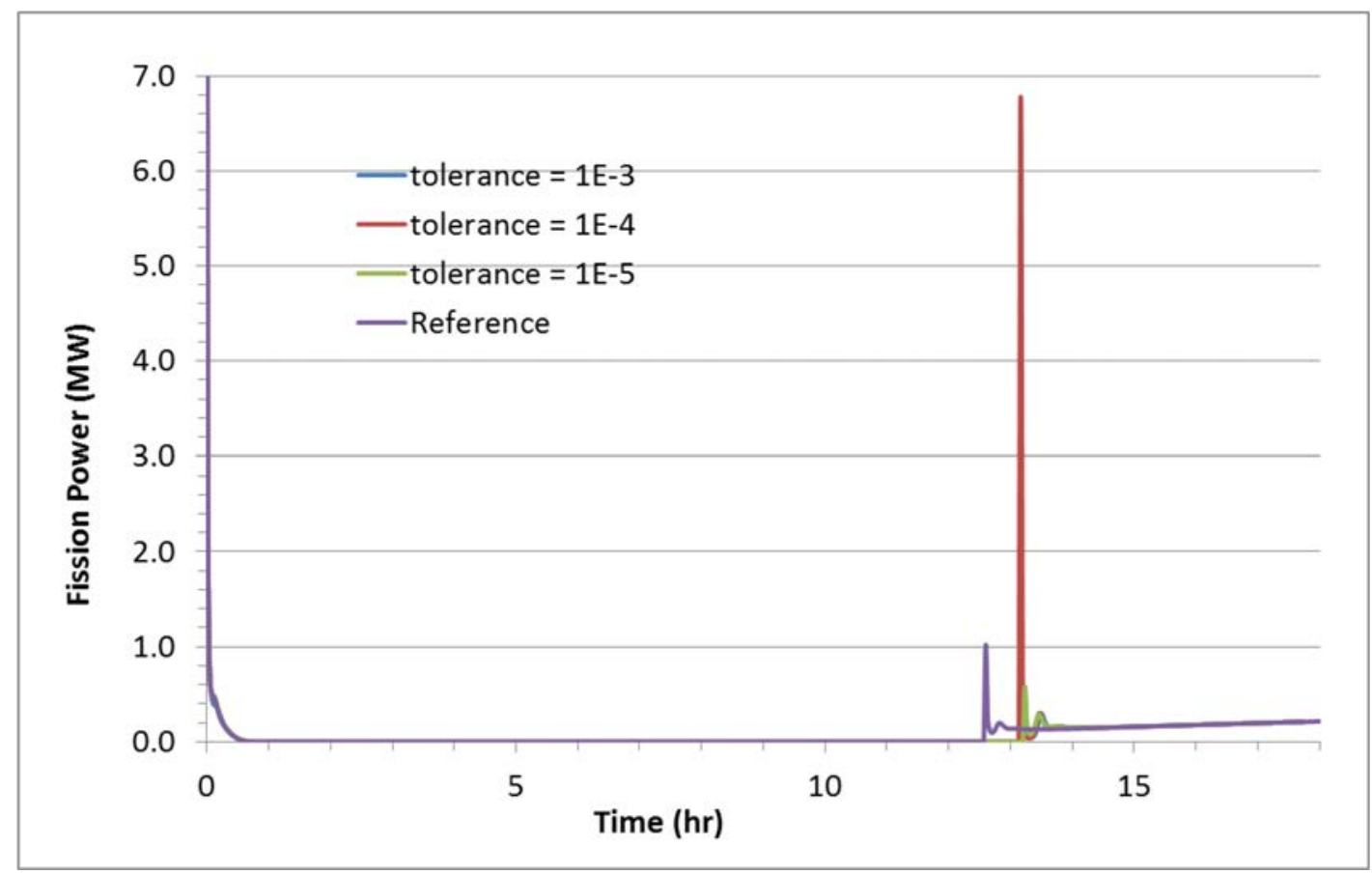

Figure 39. Fission power for the reference case and Method 1 flux1_toll using three different tolerance values: $1 \mathrm{E}-3,1 \mathrm{E}-4$, and $1 \mathrm{E}-5$.

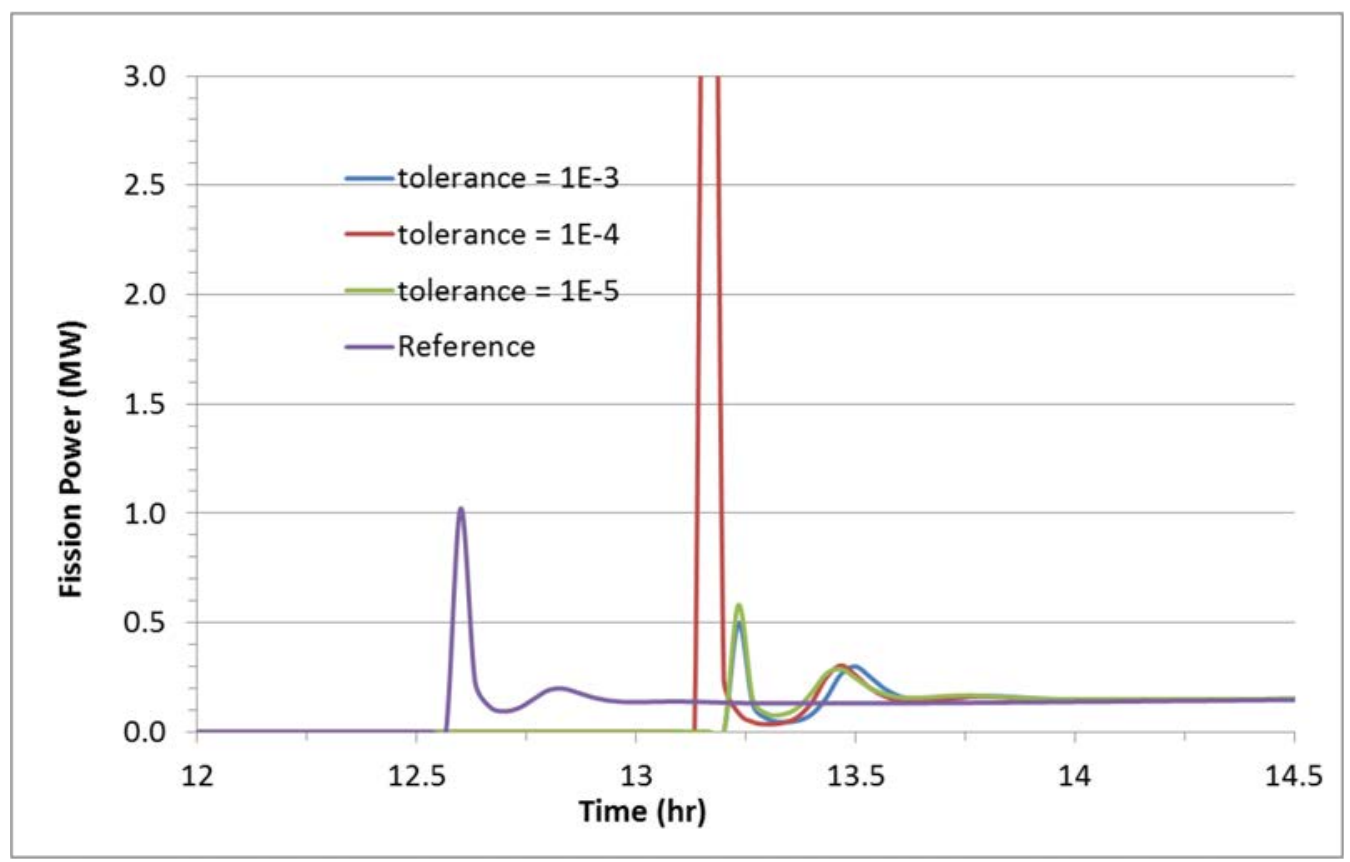

Figure 40. Fission power during the re-criticality period for the reference case and Method 1 flux 1 _toll using three different tolerance values: $1 \mathrm{E}-3,1 \mathrm{E}-4$, and $1 \mathrm{E}-5$. 


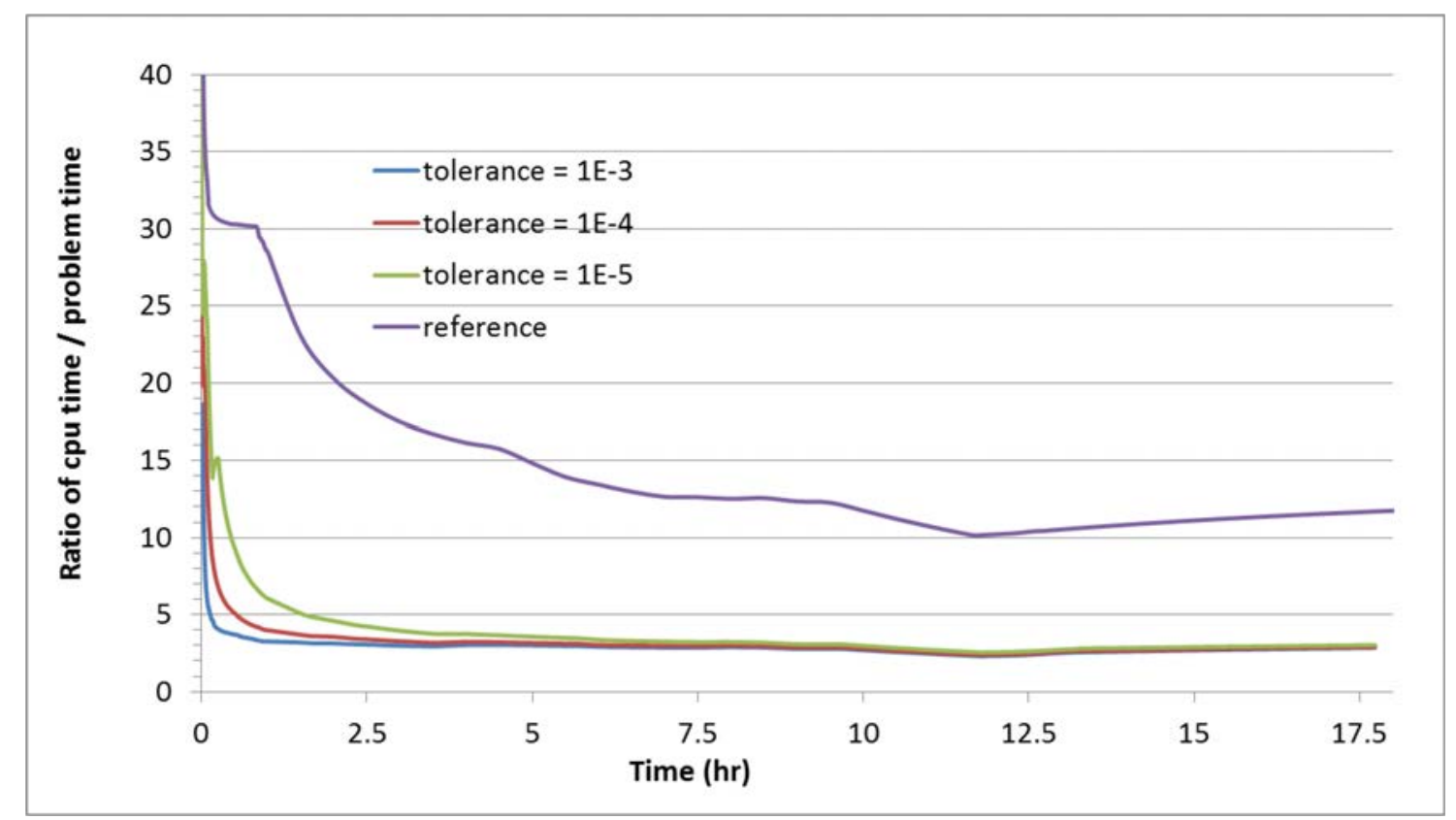

Figure 41. Time enhancement for the reference case and Method 1 using flux1_toll and three different tolerance values: $1 \mathrm{E}-3,1 \mathrm{E}-4$, and $1 \mathrm{E}-5$.

\subsubsection{Method 2 Using Maximum Time Step Between Flux_toll and Flux1_toll}

As previously mentioned, the testing matrix was set up prior to having obtained any test results for this PHISICS/RELAP5-3D HTTR model. If the data had been evaluated prior to deciding which method to test for this section on the maximum time step, Method 3 would have been preferred, which was also indicated for the 8-group testing. Results obtained using Method 2 and the maximum time step calculated using flux_toll with a tolerance of 1E-5 and flux1_toll with a tolerance of 1E-4 are seen in Figure 42.

Time enhancement associated with the calculation (Table 12 and Figure 43) remained good; however, the re-critical peak obtained was greater than the initial power level. Therefore, this method is not recommended.

Table 12. Run time summary for Method 2 using maximum time step between flux_toll (tolerance=1E-5) and flux1_toll (tolerance=1E-4).

\section{Test Case}

\section{Reference}

Method 2 with Maximum time step

between flux toll and flux 1_toll
Total CPU Time (Hours) to Simulate an 18 Hour LOFC Transient 


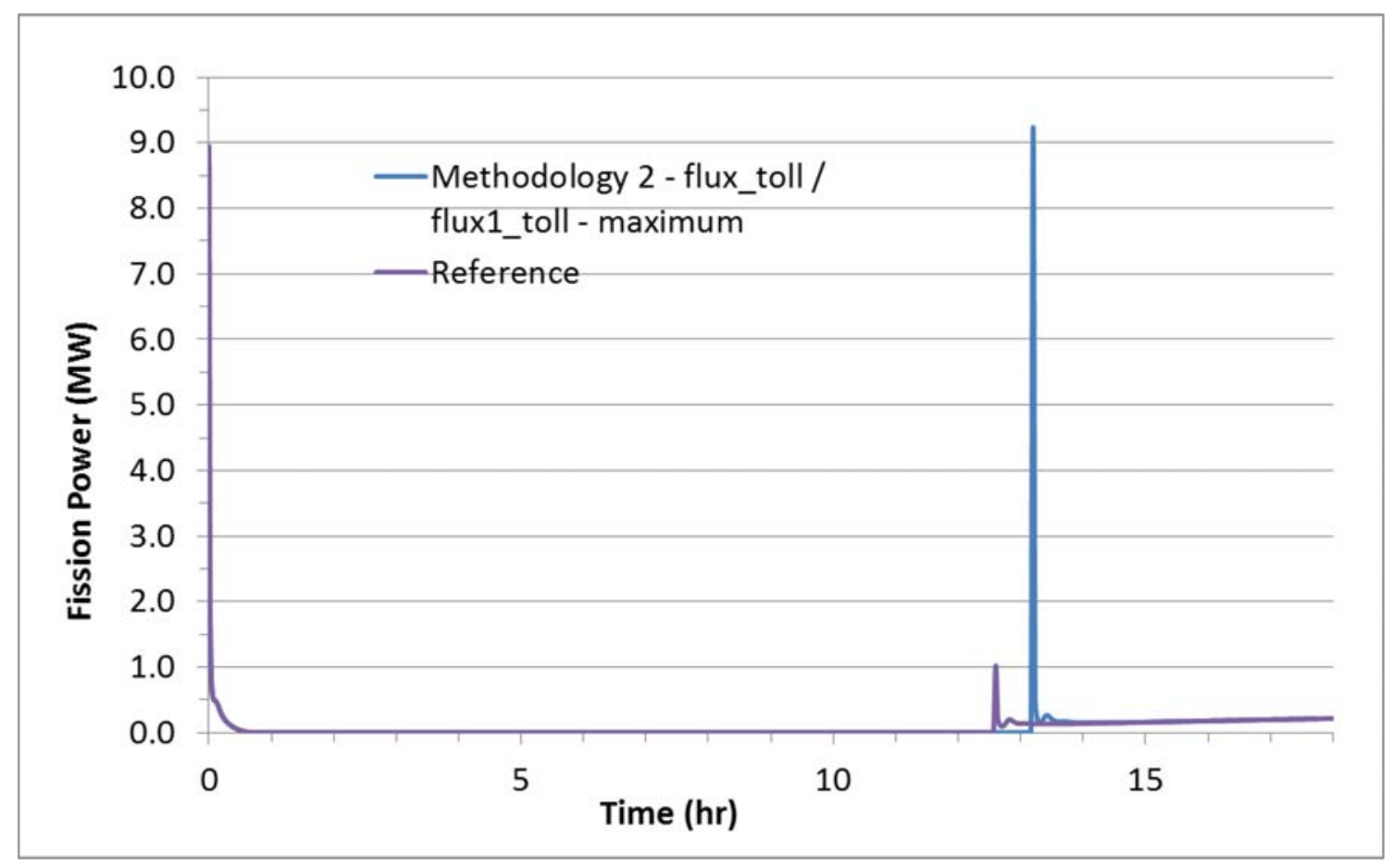

Figure 42. Fission power for the reference case and Method 2 using the maximum time step calculated using flux_toll and flux 1 _toll with tolerances of $1 \mathrm{E}-5$ and $1 \mathrm{E}-4$, respectively.

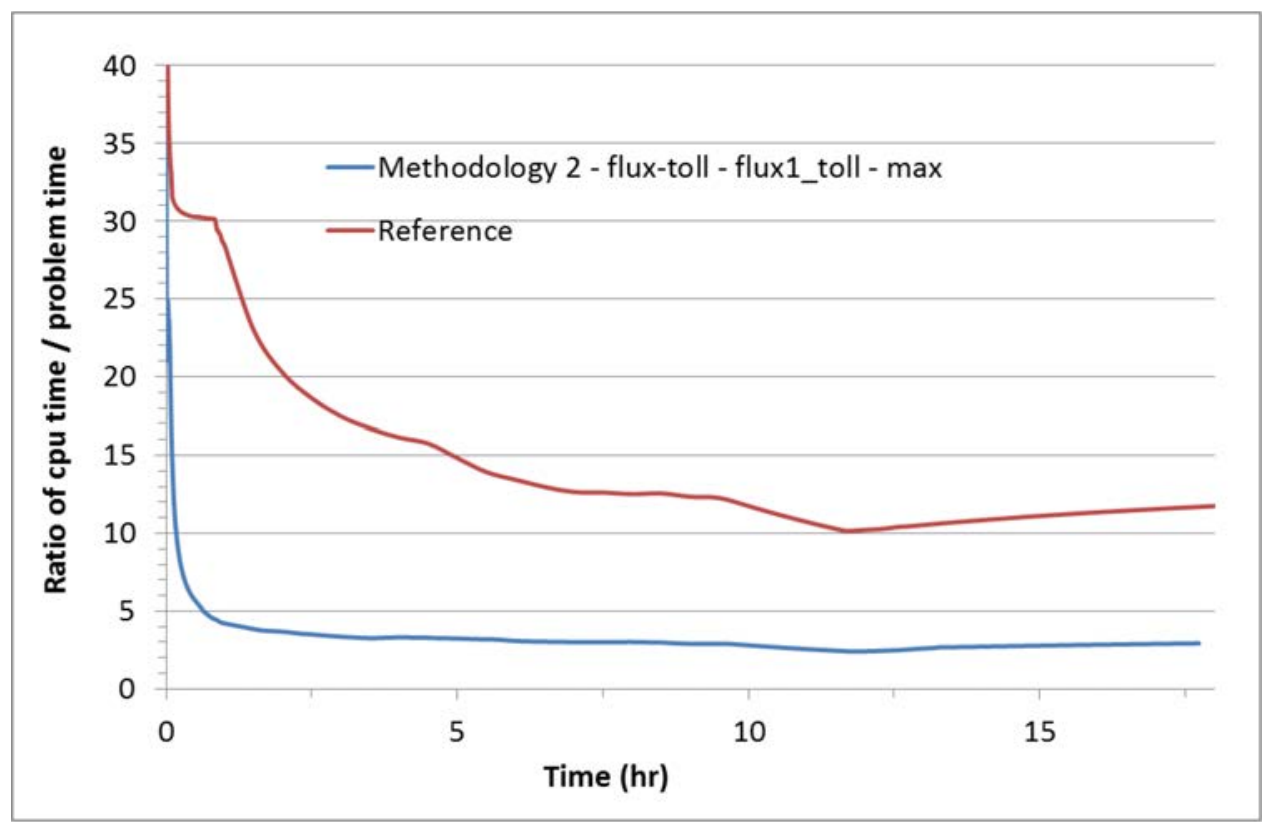

Figure 43. Time enhancement for the reference case and Method 2 using the maximum time step calculation between flux_toll and flux1_toll with tolerances of $1 \mathrm{E}-5$ and $1 \mathrm{E}-4$, respectively.

\section{CONCLUSIONS}

This report summarizes the changes made to the PHISICS-RELAP5-3D code for implementing an adaptive time-step method into the code for the first time. Various adaptive schemes, based on flux 
convergence criteria were tested using 8- and 26-energy group versions of the HTTR PHISICS/RELAP5-3D model developed by JAEA and INL for the LOFC simulation.

It was found that the adaptive method based on the absolute difference in the second derivative of the flux (flux1_toll) lead to a significant improvement in the LOFC simulation time required, without significant accuracy penalties in predictions of fission power and fuel temperature. In the 8-group model scenario, the LOFC simulation of 20 hours could be completed in real-time, or even less than real-time, compared with the previous version of the code that completed the same transient three to eight times slower than real-time.

The HTTR model is very sensitive to the choice of various combinations of solver methods and tolerance settings. Use of the adaptive method based on the relative difference in the second derivative of the flux (flux_toll) did not lead to significant simulation time gains and, in combination with some methods and tolerance settings, resulted in unacceptably high errors in some test cases. Because the adaptive time schemes introduce truncation errors into the iteration scheme, small variances in the xenon concentration levels could build-up to have a significant effect on the eventual re-critical fission power peak magnitude (but not as much on the timing of this peak). However, it should, be noted that in spite of large fission power peak differences observed for some of these test cases, fuel temperatures are only very slightly affected during the re-critical phase, because the additional energy deposited is easily absorbed by the large thermal capacity of the graphite system.

Overall, it is recommended that users of the PHISICS-RELAP5-3D HTTR model confirm the main findings of this study by using a comprehensive test matrix if any changes are made to the neutronics or thermal hydraulics input. The combination of specifying the flux1_toll method with a tolerance of 1E-3 seems to be a good starting point for future time-optimization efforts.

\section{REFERENCES}

1 Baker, R., et al., Civil Nuclear Energy Working Group Project: High Temperature Engineering Test Reactor Transient Simulation with PHISICS/RELAP5-3D, INL/LTD-16-38889, Idaho National Laboratory, 2016.

2 Balestra, P., Implementation of an Adaptive Time Step Methodology for PHISICS-RELAP5-3D Coupling Scheme, University of Rome, December 2016.

3 Turinsky, P. J., et al., NESTLE: A Few-Group Neutron Diffusion Equation Solver Utilizing the Nodal Expansion Method for Eigenvalue, Adjoint, Fixed-Source Steady-State and Transient Problems, EGG-NRE-11406, Idaho National Engineering Laboratory, June 1994.

4 Strydom, G., et. al., "Comparison of the PHISICS/RELAP5-3D Ring and Block Model Results for Phase I of the OECD/NEA MHTGR-350 Benchmark," Nuclear Technology, Vol. 193, pp. 15-35, 2015.

5 Rabiti, C., Alfonsi, A., Epiney, A., "New Simulation Schemes and Capabilities for the PHISICS/RELAP5-3D Coupled Suite," Nuclear Science and Engineering, Vol. 182, No. 1, pp. 104$118,2015$. 


\section{Appendix A}

\section{HTTR and the PHISICS-RELAP5-3D Model}




\section{Appendix A HTTR and the PHISICS-RELAP5-3D Model}

A description of the HTTR and the RELAP5-3D model of the HTTR is provided in this Appendix. The information provided here was taken from a report issued in December 2016 which had a limited distribution due to proprietary data. The proprietary data is not included here.

\section{HIGH TEMPERATURE ENGINEERING TEST REACTOR DESCRIPTION}

Located in Japan at the Oarai Research and Development Center of JAEA, the HTTR is a $30 \mathrm{MWt}$-rated, helium-cooled and graphite moderated prismatic block HTGR. The first criticality of the HTTR was achieved in November 1998. A detailed description of the HTTR is given in Reference 1.

The LOFC testing was performed at a starting power level of $30 \%$ or $9 \mathrm{MW}$. Table A-1 lists operating parameters for the HTTR at $9 \mathrm{MW}$.

Table A-1. High Temperature Engineering Test Reactor design parameters at $9 \mathrm{MW}{ }^{2}$

\begin{tabular}{|l|l|}
\hline Coolant & Helium \\
\hline Outlet coolant temperature & $320^{\circ} \mathrm{C}$ \\
\hline Inlet coolant temperature & $180^{\circ} \mathrm{C}$ \\
\hline Primary pressure & $2.774 \mathrm{MPa}$ \\
\hline Average power density & $2.5 \mathrm{~W} / \mathrm{cm}^{3}$ \\
\hline Core diameter & $2.9 \mathrm{~m}$ \\
\hline Fuel & Low enriched uranium $(3-10 \%)$ \\
\hline
\end{tabular}

Important HTTR design features are summarized in the following sections.

\subsection{Fuel Design}

The HTTR fuel blocks ${ }^{3}$ are a prismatic pin-in-block type design, such that the helium flow channels in the graphite block also contain the fuel rods. The annular fuel rods consist of 14 stacked fuel compacts. Each compact is cylindrically shaped, with a central region that is filled with stagnant helium when the fuel rod is assembled. The fuel compacts consist of low-enriched uranium dioxide $\left(\mathrm{UO}_{2}\right)$ kernels coated with multiple layers of high-density pyrolytic carbon and silicon carbide layers in a form known as tristructural isotropic (TRISO) fuel, embedded in a graphite matrix. The fuel rods are encased in an outer graphite sleeve, with a helium gap between the sleeve and compact. The fuel rods are then loaded into the channels of the fuel block as shown in Figure A-1. Each fuel block also contains two burnable poison (BP) rods, each located in a vertex of the block for excess reactivity control. Fuel block specifications are contained in Table A-2. 


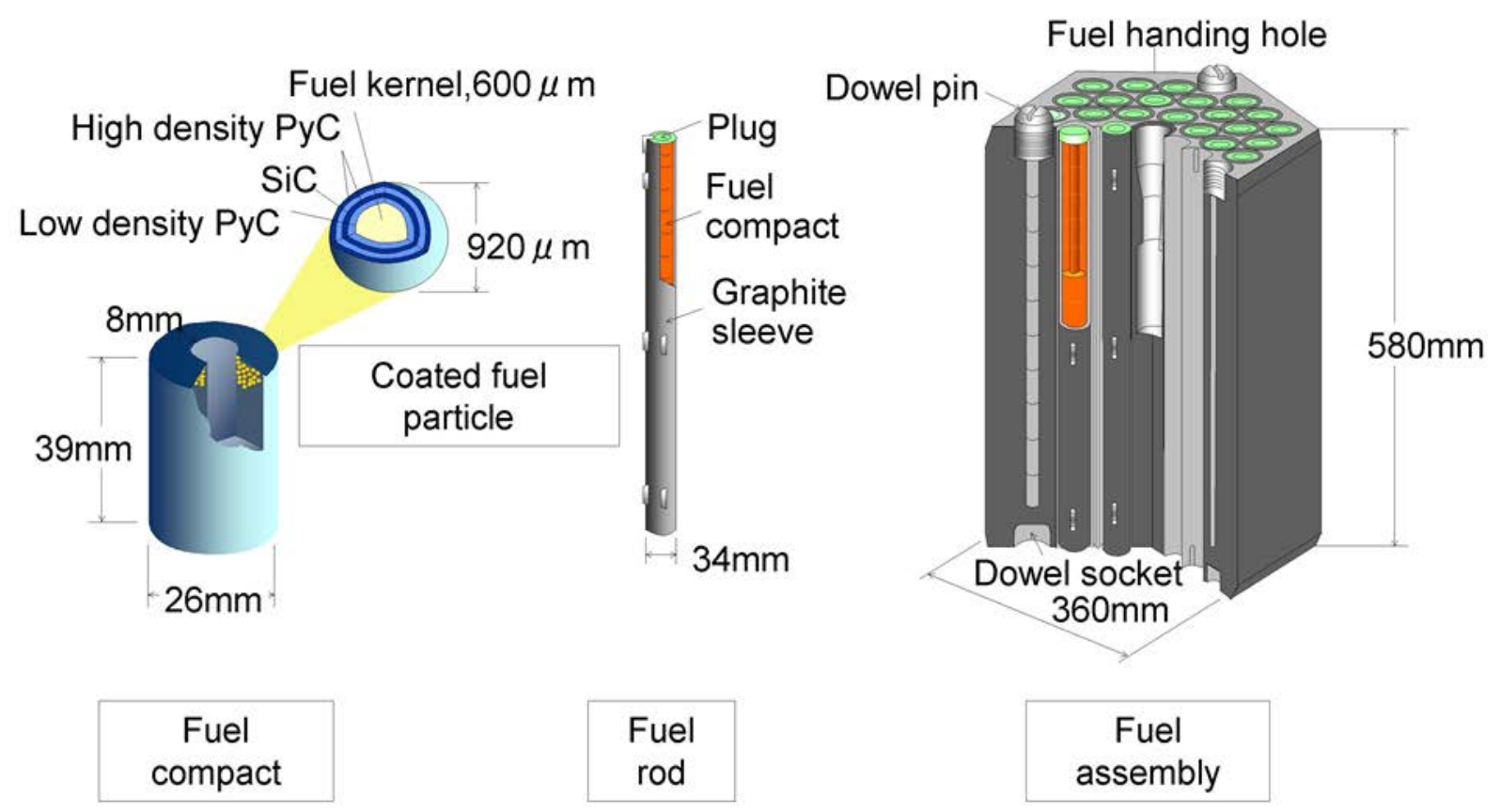

Figure A-1. Cutaway of a HTTR compact and prismatic fuel block. ${ }^{3}$ 
Table A-2. Fuel block and fuel compact specifications. ${ }^{3}$

\begin{tabular}{|l|l|}
\hline Fuel Block & Pin in block \\
\hline Design & Hexagonal \\
\hline Shape & - \\
\hline Dimension & $0.58 \mathrm{~m}$ \\
\hline Height & $0.36 \mathrm{~m}$ \\
\hline Flat-to-flat width & $0.041 \mathrm{~m}$ \\
\hline Fuel hole diameter & Graphite \\
\hline Material & 33 \\
\hline Number of fuel rods - Ring 1 or 2 & 31 \\
\hline Number of fuel rods - Ring 3 & \\
\hline Fuel Compact & Hollow cylinder \\
\hline Design & - \\
\hline Dimension & $0.026 / 0.010 \mathrm{~m}$ \\
\hline Outer/inner diameter & $0.039 \mathrm{~m}$ \\
\hline Height of a compact & $0.546 \mathrm{~m} \mathrm{(14} \mathrm{fuel} \mathrm{compacts)}$ \\
\hline Effective length of fuel rod & \\
\hline Graphite Sleeve & Cylindrical \\
\hline Design & - \\
\hline Dimensions & $0.034 \mathrm{~m}$ \\
\hline Outer diameter & 0.00375 \\
\hline Thickness & $0.580 \mathrm{~m}$ \\
\hline Length & $0.00025 \mathrm{~m}$ \\
\hline Gap between fuel and graphite sleeve (helium gap) & \\
\hline
\end{tabular}

\subsection{Core Layout}

The HTTR core consists of concentric radial rings of fuel and absorber (control) blocks surrounded by permanent graphite reflector blocks; a top view of which is illustrated in Figure A-2. The three types of prismatic blocks are fuel, control rod, and reflector blocks, each with the same outer dimensions. Rings 1, 2, and 3 contain the fuel, Ring 4 is the side reflector, and Ring 5 is the permanent reflector. The (radial) center block is a control rod (CR) block.

The CR blocks have three holes: two for the control rods and one for the reserve shutdown system in case an additional shutdown method is required. Seven pairs of CRs are in the core (including center CR rod block and Rings 1-3) and nine pairs of CRs are in the side reflector. Three of the pairs of CRs in the side reflector are for shutdown margin and the other six pairs are inserted with the remaining CRs to control reactivity and maintain a relatively flat radial power.

The active core (center CR, and Rings 1, 2, and 3) comprise as mixture of fuel and CR blocks, and a cross-section is shown in Figure A-3 on the left. Ring 1 (surrounding and adjacent to the central absorber block) and Ring 2 each consists of 6 fuel blocks containing 33 fuel rods/flow holes in each block. Ring 2 also contains six CR blocks alternating with the fuel blocks. Ring 3 contains 18 fuel blocks, each with 31 fuel/flow holes. Ring 4 consists of nine CR blocks and 15 graphite reflector blocks comprising the replaceable inner side reflector. The graphite reflector blocks in Ring 4 do not contain any flow holes. 
Surrounding Ring 4 is the permanent reflector. These graphite blocks are not replaced during the life of the reactor. They are larger than the prismatic blocks and provide structural support to the core in addition to being reflectors. Between the reactor pressure vessel (RPV) and the permanent reflector is the side shield that protects the RPV inner wall from neutron embrittlement.

Axially, there are two layers of graphite reflector blocks above and below the core region. These blocks contain flow holes that align with the fuel blocks. Each block has three dowels on the upper face and three sockets on the lower face to ensure the blocks and associated flow holes remain in alignment for cooling. Above the uppermost reflector block is the upper shield, which has flow holes as well, and helium flows through these from the upper plenum to the lower plenum. There are five fuel blocks between the upper and lower reflector blocks. The fuel enrichment of the fuel blocks varies both radially and axially within the core.

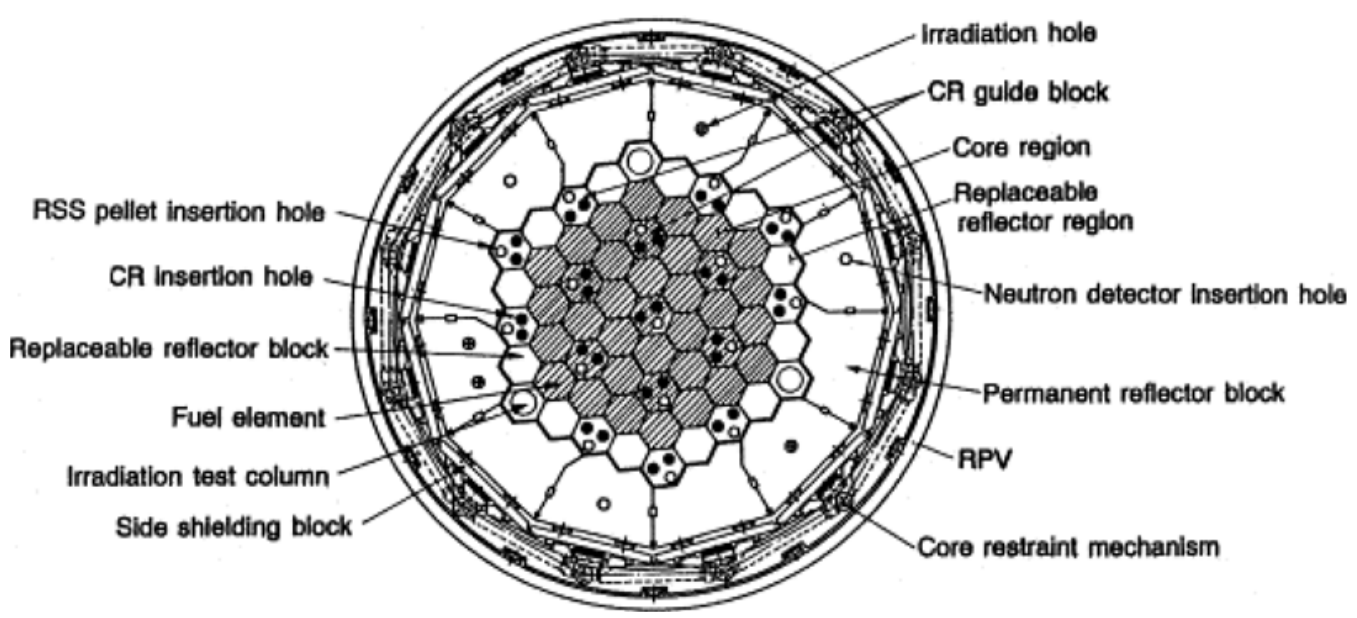

Figure A-2. Horizontal arrangement of the core. ${ }^{4}$

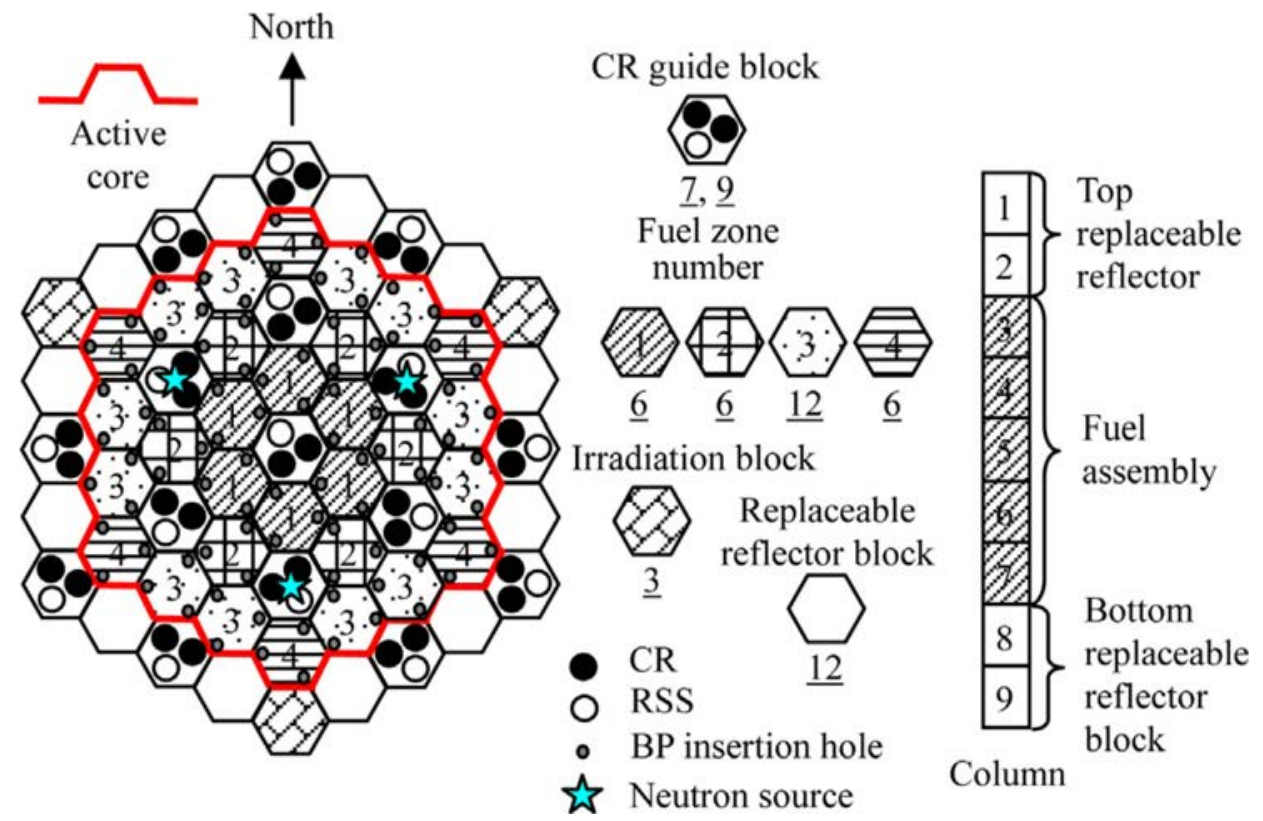

Figure A-3. Core arrangement of the HTTR. The underlined numbers (e.g., 6, 6, 12, and 6 under the different type of fuel elements) denote the total number of blocks. ${ }^{5}$ 


\subsection{Flow Paths}

Helium flows into and out of the bottom of the RPV through concentric pipes, with the hot gas leaving through the inner pipe. Inflow is via the outer annulus, into the lower plenum, and up through parallel paths - one between the RPV and side shielding and the other between the shielding and the permanent reflector. The flow converges in the upper plenum, where it is then directed downward through the engineered parallel paths in reactor internal structures. The helium is propelled down through the upper shield, upper reflectors, fuel blocks, CR blocks, gaps between the blocks, side reflector, permanent reflector, and lower reflectors. The coolant emerges into the outlet plenum, where the various flow paths converge once again and continue past the key blocks and seal blocks that form the high temperature plenum, and finally, out through the center region of the concentric high temperature pipe. Figure A-4 shows a cut away view of the RPV with arrows indicating the normal flow path. Bypass flow, which is the flow between the hexagonal blocks, is estimated to range from $3 \%$ to $10 \%$ and will vary over core life.

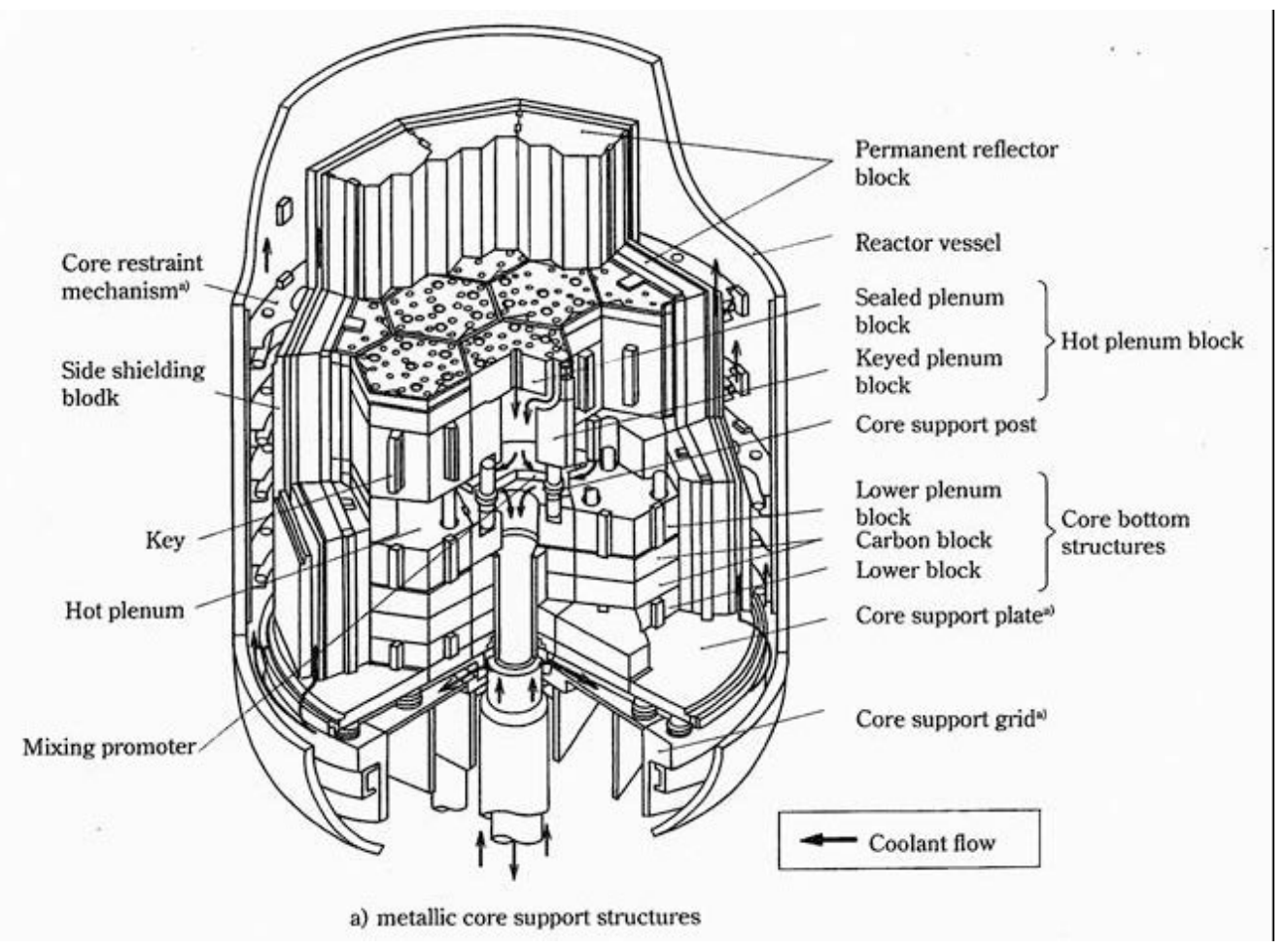

Figure A-4. Reactor core internals of the High Temperature Engineering Test Reactor. ${ }^{6}$

Helium flow outside of the RPV is part of the main cooling system. The components associated with this system, as well as the auxiliary cooling system, are not described within this report because they do not play a significant role in either the steady state or the transient conditions included in this study. Figure A-5 shows an axial slice of the RPV along with arrows indicating flow paths. 


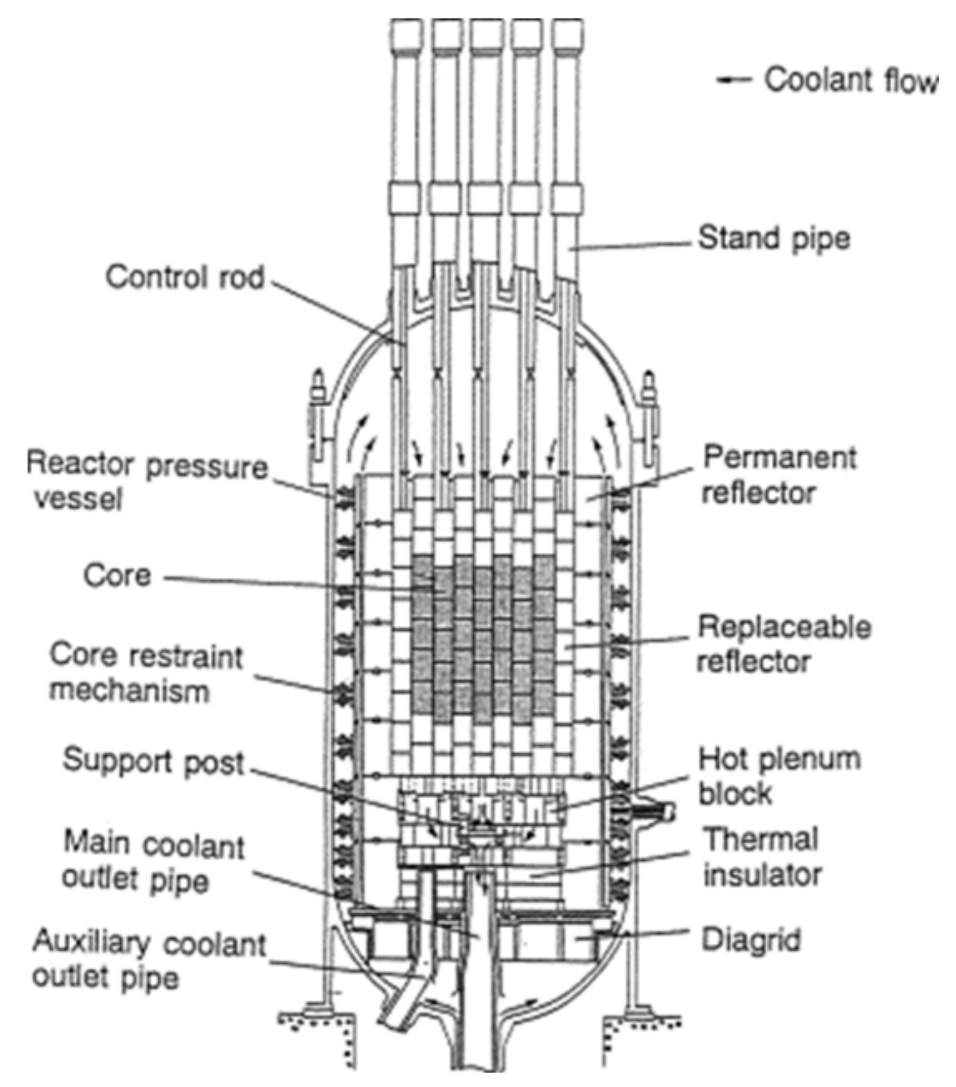

Figure A-5. Vertical view of High Temperature Engineering Test Reactor. ${ }^{3}$

\subsection{Heat Removal}

Outside of the RPV, heat removal is performed by the two independent vessel cooling systems (VCSs), which are among the engineered safety features (ESFs) of the plant. ${ }^{7}$ The VCS consists of cooling panels located around the sides of the RPV, as well as above and below the vessel, as shown in Figure A-6. The hot pressure vessel radiates heat to the VCS panels that conduct the heat to the cooling water inside. Under normal operation, coolant pumps circulate the water to a cooler that maintains the panel inlet coolant temperature less than $298 \mathrm{~K}$. The cooler transfers the heat to a secondary water coolant that then rejects the heat to the atmosphere via an air cooler.

Under a loss of plant power event, the VCS loops operate by natural circulation at a sufficient rate to maintain pressure vessel integrity (as is to be demonstrated in the planned OEDC-NEA LOFC experiments). This ESF system is a dual-train system that is designed to remove heat in the event that normal cooling via helium is not available. During normal operation, the VCS also removes heat from the concrete biological shield. Within the reactor vessel and during normal operation, heat removal is primarily through convection by the forced flow of helium through the reactor. During the LOFC transient, once the helium flow is secured, conduction and radiation heat transfer are the dominant means of heat transfer. 


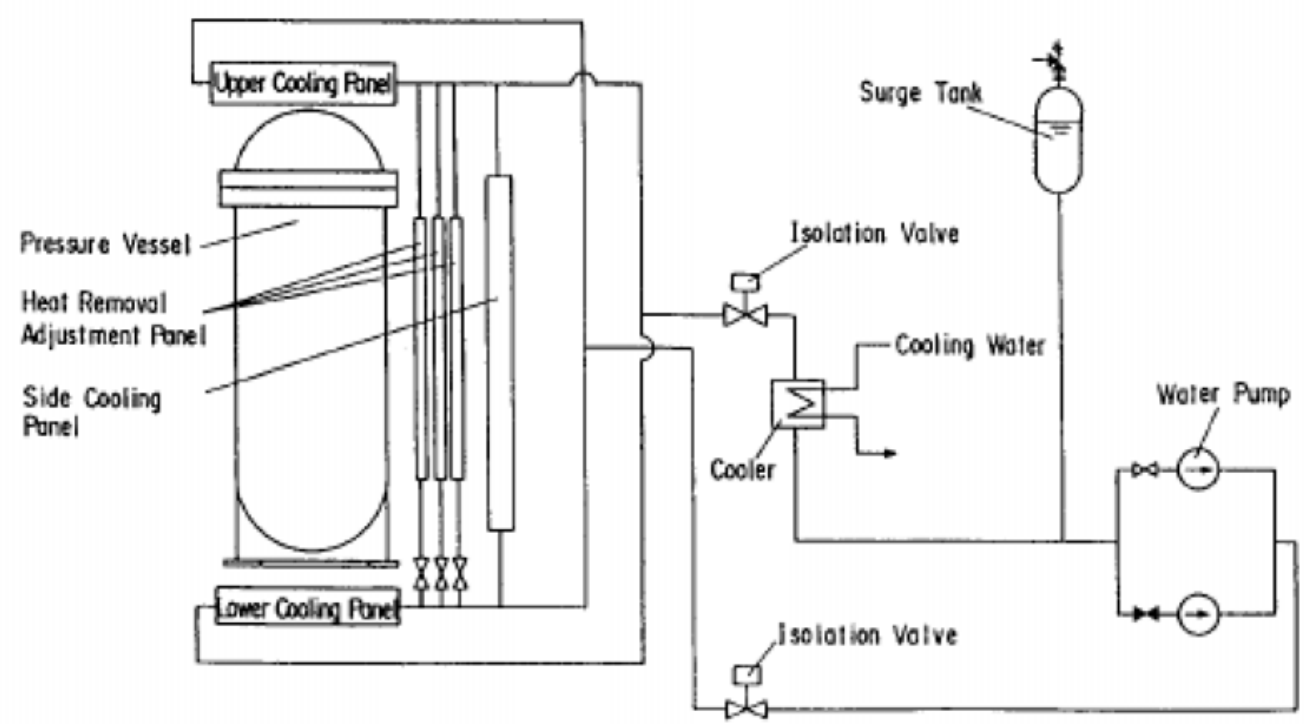

Figure A-6. Flow sheet of the HTTR vessel cooling system. ${ }^{7}$

\section{PHISICS/RELAP5-3D MODEL DESCRIPTION \\ 2.1 Modeling}

Multiple approaches are possible when using RELAP5-3D to model the operation of a reactor. Analyzing flow paths and dividing the reactor into major components to be modeled for thermal fluids analysis is required for every approach. The components and flow paths are then broken down into a nodalization scheme, such that the phenomena of interest can be adequately simulated. Heat transfer calculations via conduction, convection, and radiation are included in the model. Kinetics calculations performed by RELAP5-3D may be performed using point or nodal kinetics (limited in the NESTLE module to two- or four-neutron energy groups). PHISICS is coupled with RELAP5-3D to perform transport calculations with as many neutron energy groups as desired.

For this project, RELAP5-3D Version 4.3.4 was used, which was the most current version of the code available at the time. The major primary system flow paths and structures in the reactor vessel are included in the HTTR input deck; however, secondary systems external to the reactor, with the exception of the VCS, are not included. A more detailed description of the modeling is provided in Section 2.2. A summary of the flow components and heat structures defined in the input deck for RELAP5-3D is provided in Appendix A (of original document; not included in this excerpt). JAEA, with support from INL, produced the RELAP5-3D input deck to model the HTTR, but subsequent revisions of the original JAEA RELAP5-3D model have been implemented since October 2015.

The major sections of the RELAP5-3D input model include:

- Fluid dynamics components specifying the flow paths of helium through the reactor

- Heat structures, including the fuel, graphite, and control rod blocks, upper and lower reflectors, side and permanent reflectors, side shield, inlet and outlet plenum, reactor vessel and VCS

- Conduction and radiation enclosures linking heat structures for conductive and radiative heat transfer

- A kinetics section defining heat structures interfacing with the neutronics calculations

- Tabulated material properties. 
The axial and radial structures and flow paths are nodalized such that sufficiently detailed power, flux, and temperature profiles may be obtained. A one-third core neutronics model was used for calculation time efficiency. More specific modeling information is provided in the following sections.

\subsection{Unit Cell Model}

Graphite blocks in the RELAP5-3D model were constructed by repeating unit cells. The unit cell model uses cylindrical geometry and describes each of the HTTR rings for each axial level defined. The $\mathrm{CR}$, fuel, and graphite blocks each use the unit cell concept, and due to variations in the blocks geometry (e.g., control rod holes, 31 versus 33 flow holes, number of fuel blocks), the resultant definitions in RELAP5-3D vary. The unit cell concept for each of these block types will be described.

The center CR block was modeled as two unit cells. Although there are three holes in the control rod blocks, only two actually have a flow path (the reserve shutdown system (RSS) channel is closed at the bottom to hold the poison spheres). Figure A-7(a) represents the top view of the CR block and Figure A-7(b) is the view showing the modeled view of the CR block. The unit cell, Figure A-7(c), consists of a coolant channel surrounded by a graphite heat structure; two unit cells per CR block. The unit cells were modeled using the actual value of the inside radius of the CR channel surrounded by an annulus of graphite sufficient to preserve the total cross sectional area of the block. The total cross sectional surface area of graphite in the block did account for the RSS hole, but did not exclude the area of the handling hole and the burnable poison pins. The graphite area per unit cell is the total area of the block divided by the number of unit cells in the block. The graphite radius is determined using Equation (A-1).

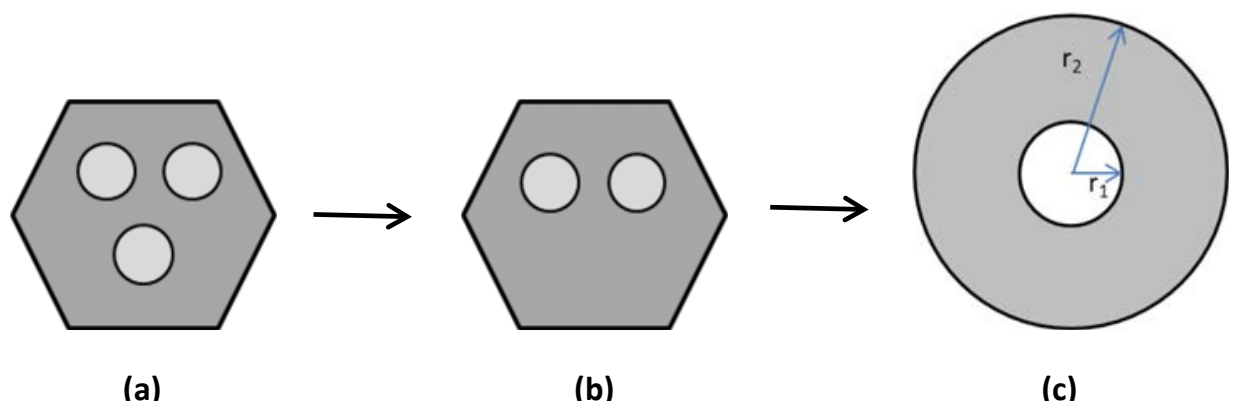

Figure A-7. Control rod block unit cell.

total graphite area per unit cell $=\pi\left(\mathrm{r}_{\text {graphite }}^{2}-\mathrm{r}_{\text {flow area }}^{2}\right)$

The radius of the control rod channel changes axially which requires the equivalent graphite radius to be recalculated as the geometry changes. The same process is used for the CR blocks in Rings 2 and 4. The difference for Ring 2 is that there are six blocks with CRs and each block will be modeled with two unit cells; thus, a total of $12 \mathrm{CR}$ unit cells for Ring 2 are modeled. Ring 4 is modeled such that the blocks are divided in half radially. Half of the CR blocks are modeled as part of the inner Ring 4, and the other half of the CR blocks are modeled in the outer Ring 4. Because there are nine CR blocks, each half will consist of nine CR unit cells.

The solid-gas interface surface area in a unit cell model of the block often differs from actual block surface area. To preserve the rate of convective heat transfer from the block into the coolant, a factor is applied to the heat transfer coefficient to correct for the difference. This correction factor is input as a multiplier on the convective heat transfer coefficient, and is calculated using Equation (A-2).

Multiplier $=\frac{\text { Actual side surface area }}{\text { Modeled side surface area }}$ 
Ring 1 contains six blocks. Axially, from the inlet to the outlet plenums, there are 10 blocks: one upper shield block, two upper reflector blocks, five fuel blocks, and two lower reflector blocks. The fuel blocks are modeled with two unit cells, one for fuel and one for graphite to allow separate tracking of the fuel and moderator temperature feedbacks to the kinetics. Figure A-8(a) represents both the fuel and the graphite portions of the unit cell; however, these are separately modeled within RELAP5-3D. The fuel unit cell model, Figure A-8(b), includes the fuel rod $\left(r_{2}\right)$, surrounded by a graphite sleeve $\left(r_{3}\right)$ with a helium gap in between (the helium gap is not represented in the figure). These materials are modeled such that actual geometry is matched in the model. The hollow center (stagnant helium) of the fuel compact $\left(r_{1}\right)$ is included in the model. Helium flow is modeled on the outer perimeter $\left(r_{4}\right)$ of the unit cell. The total number of fuel unit cells is equal to the number of fuel rods per block, multiplied by the number of blocks with fuel (for Ring 1, 33 ×6 = 198 fuel unit cells). The regions without fuel are modeled with a unit cell with graphite only as represented by Figure A-8(c). These blocks have 33 flow holes for each axial level except for the lowest reflector block which has six holes for combining flow. Therefore, each block contains 33 unit cells (six unit cells in lower reflector 2). Equation (A-1)) is used to find the equivalent outer graphite radius, $r_{2}$ in Figure A-8(c), for the graphite block heat structure with the actual coolant channel outer radius, $r_{1}$ in Figure A-8(c), (which varies axially) used as the inner radius. Helium flow is modeled on the inner surface of the unit cell. Equation (A-2) is used to calculate the heat transfer coefficient multiplier for the outside of the heat structure.
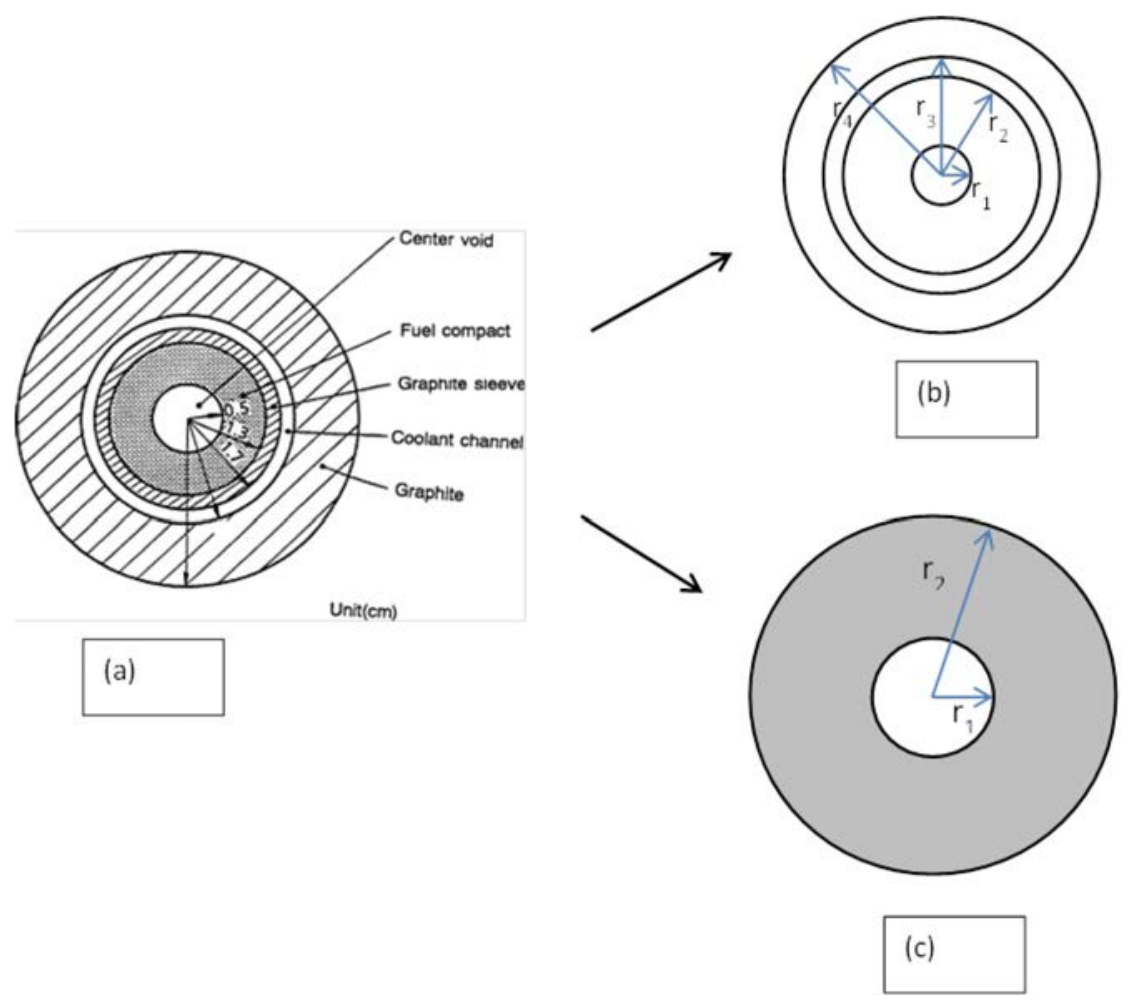

Figure A-8. Fuel block unit cell. ${ }^{5}$

Ring 2 contains a total of 12 blocks; six control rod blocks that are modeled like the center CR block and six blocks that are modeled like Ring 1 blocks.

Ring 3 contains 18 blocks that are modeled in a way similar to Ring 1. For these blocks, there are 31 fuel pins per block, which equates to 31 unit cells per block. The total number of unit cells in Ring 3 would then be the number of unit cells multiplied by the total number of blocks $(31 * 18=558)$. 
The 24 blocks in Ring 4 consist of two types shown in Figure A-9; 12 CR blocks (a) and 12 solid graphite blocks (b). As previously mentioned, for modeling purposes, Ring 4 blocks are divided in half radially (Figure A-10(b)), and the blocks are modeled as CR blocks (Figure A-10(a)). Three of the 12 CR blocks (of the same design as CR blocks) are actually called irradiation blocks, but were modeled in RELAP5-3D as solid blocks. The nine CR blocks were modeled as nine unit cells for each half of the Ring 4 blocks, using the same guidance as the center CR block.

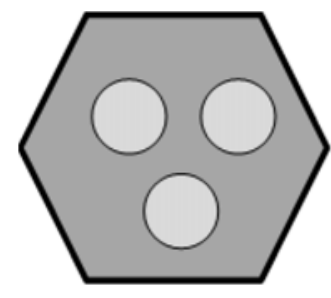

(a)

Figure A-9. Ring 4, side reflector block types.

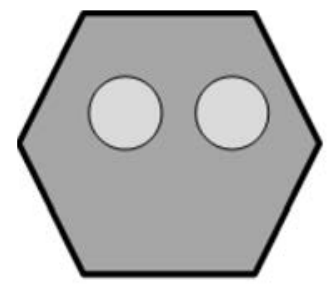

(a)

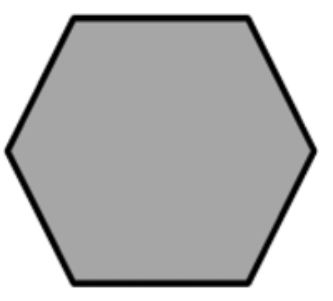

(b)

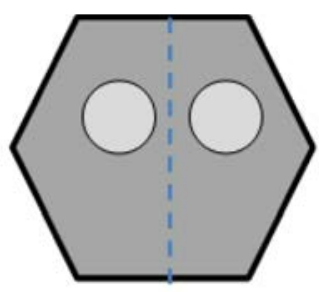

(b)

Figure A-10. Ring 4 block representation for the unit cell model.

Ring 5, the permanent reflector ring, and the side shield surrounding the permanent reflector are modeled based on actual dimensions.

The axial nodalization for the LOFC transient and the power ramp transient (see Section 8 of the original document; not included in this excerpt) differ slightly to support their individual modeling needs. For the LOFC model, the axial extent between the upper plenum/core inlet and the core outlet is divided into 15 nodes with one in the upper shield, two in the upper reflectors, 10 in the fuel (each block was divided into two nodes), and two in the lower reflectors. This axial discretization is maintained for all components between these elevations. A RELAP5-3D heat structure requires that the geometry and materials be constant over the length of the structure. The different coolant channel radii, fuel, and reflector materials will not allow fewer structures to be used. The fuel enrichment for each of the rings varies both radially and axially as shown in Table 4 (of the original document; not included in this excerpt). The outer shield, RPV, and VCS include additional nodes above and/or below these 15 layers to enable the modeling of heat transfer in the upper and lower regions of the RPV. A summary of the heat structures used in the RELAP5-3D modeling is included in Appendix A (of the original document; not included in this excerpt).

The power ramp model differs in axial nodalization in that the top two fuel blocks were divided into sixths, supporting refined CR insertion points and resulting in a total node count of 23 . This increased number of nodes in the upper two fuel blocks required some heat structures and volumes to be matched with the new top fuel block axial nodalization which required additional changes to be made to support the added nodes. 


\subsection{Flow Paths}

Flow paths were modeled to include all major pathways from entry to exit from the RPV. Sections of flow were chosen based on core geometry configuration, as well as structures with which the flow is coming into contact. Helium flow was modeled from entry through the annulus of the high temperature pipe, into the lower plenum, side annulus, and upper plenum, down through the core via multiple parallel paths, into the high temperature plenum, and out through the center of the high temperature pipe. The multiple parallel paths represent helium through the fuel channels, control rod blocks, and gaps between the prismatic blocks (referred to as bypass flow). These flows join in the high temperature plenum.

The VCS model consists of panels located around the outside of the RPV, which are cooled by circulating water and air natural circulation. The circulating water system supporting the VCS is not modeled. A defined boundary condition is that the VCS will maintain a constant temperature of $333 \mathrm{~K}$ on the side opposite from the RPV.

\subsection{Heat Transfer - Conduction and Radiation Enclosures}

Besides convection, the principal heat transfer mechanisms that need to be modeled in the RPV are axial conduction within the structures and radiation between the fuel and graphite and between blocks in adjacent rings. Normally, the axial conduction would be handled internally within the heat structures, allowing the radiation to be modeled using the two available surfaces (left and right) of the heat structures. However, fluence-dependent thermal properties require that the axial stacks of blocks be divided into multiple different heat structures. This, in turn, requires that conduction enclosures must be used to model the axial conduction. Since a heat structure surface can be modeled as part of either a conduction or radiation enclosure, but not both, a different approach must be used to model both radiation and conduction. An additional heat structure is used to do this, and is referred to as the "sheath."

The sheath is a very thin $(1 \mathrm{~mm})$ layer of graphite on the surface of each fuel block, at each axial level. The sheath is the same material as the graphite block and is modeled as thin as possible around the graphite block to ensure its temperature is as close as possible to the block surface temperature. This heat structure allows modeling of the axial conduction between the graphite blocks, as well as radial radiation between the fuel and the graphite and between rings. The sheath was implemented as part of this model as a relatively new concept in the modeling used by INL. Due to the fact that the sheath is very thin, small time increments of 0.02 seconds are required during the LOFC simulation to ensure PHISICS/RELAP5$3 \mathrm{D}$ convergence. This resulted in very long run times to model the LOFC transient.

A non-sheath model of the HTTR was, therefore, also developed to support a faster calculation time for the LOFC and tritium power ramp models, utilizing a larger time step. As part of this model, the separate heat structures for each of the fuel blocks were combined as one, eliminating the need for the conduction enclosure model for the fuel blocks. The axial conduction is modeled directly by the heat structure input cards. The conduction and radiation enclosures maintain the heat transfer mechanisms modeled in the "sheath" model for the LOFC model. For the tritium power ramp model, which also contains an increased number of axial levels, the radiation enclosure model does not include all of the heat structures; however, because this model is intended to be used for modeling where forced flow was being maintained, convection would be the main heat transfer mechanism, and the heat transfer modeled in this enclosure was not needed.

A 2D representation of an axial slice of the HTTR (one-half of the slice) is shown in Figure A-11, with many of the heat structures modeled included. The left side represents the reactor center, and the right side, the VCS. The top area is outside the RPV head and the bottom is the outlet flow path of the helium. To model the heat transfer radially via conduction and radiation, the heat structures must be included in the conduction and radiation enclosures. The coolant flow to the inlet plenum is shown, but the various flow paths down through the fuel blocks, CR blocks, and bypasses between the blocks are not represented. 
Bypass flows between the blocks are modeled as passing on the right surface of the sheath, preserving the concept of the sheath being a layer of graphite around the fuel block. The outer surface of the graphite block is modeled to conduct radially with the sheath heat structure. The inner side of the graphite block is connected with the fuel region via a radiation enclosure.

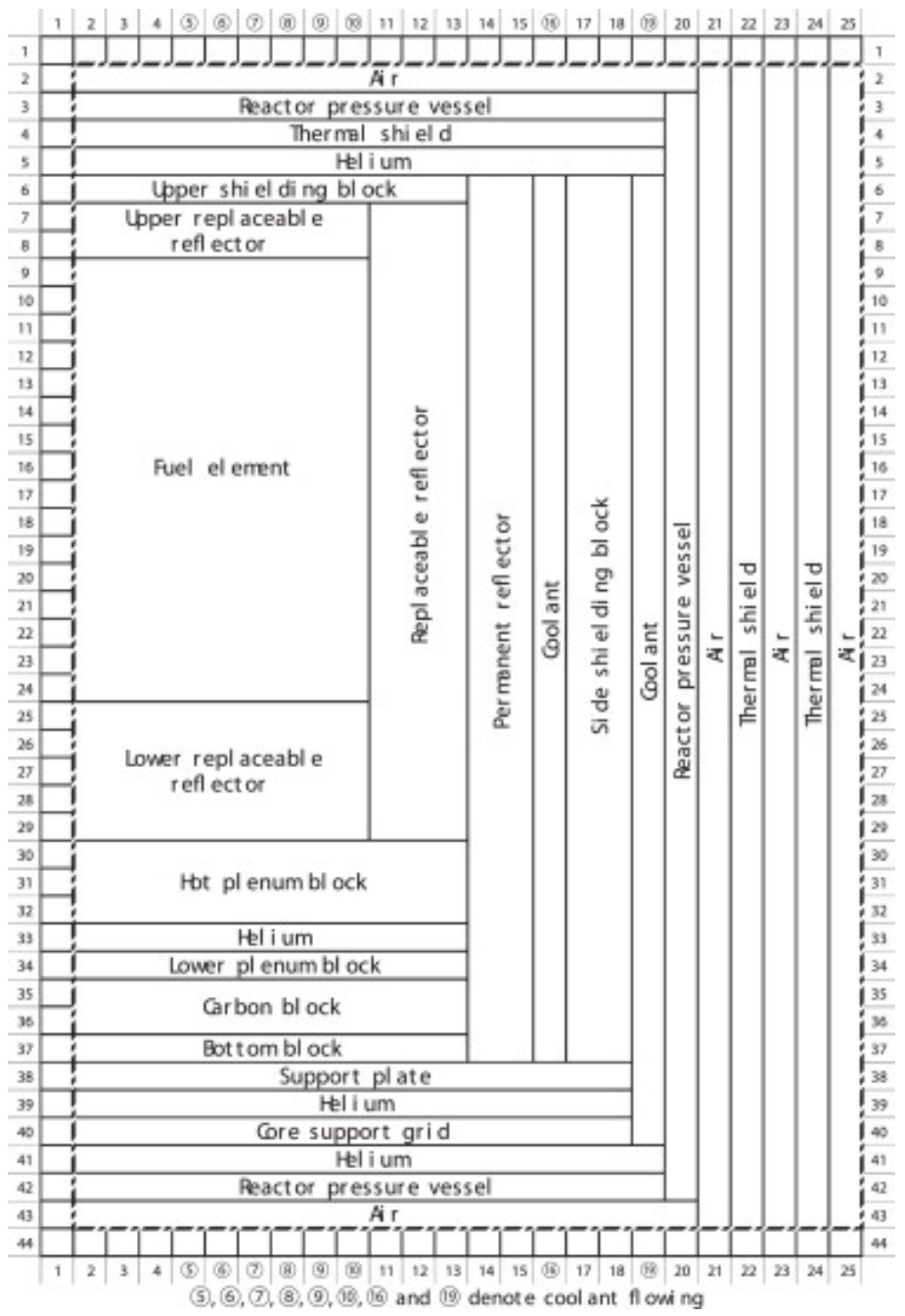

Figure A-11. Analytical model of the HTTR core. ${ }^{5}$

When forced cooling flow is present, convection heat transfer will occur on the outer side of the sheath (from the small amount of bypass flow) and on the outside of the fuel/inside of the graphite block. Once flow is lost, convection heat transfer is not as significant as conduction and radiation. The sheath will radiate from its right side (bypass flow side) to other sheaths and/or graphite blocks around it. The outer side of the fuel will radiate to the inner side of the graphite block. The outer side of the graphite block conducts radially with the sheath, and axially with the block above or below.

\subsection{Kinetics - PHISICS Coupling}

The spatial modeling of the reactor neutronics feedback is essential for accurate overall reactor response during a transient such as the re-critical LOFC (which is the first target application of this model). The feedback of the fuel and graphite/moderator temperatures to the PHISICS module is specified in the kinetics section of the RELAP5-3D input model. Heat structures coupled to PHISICS for the feedback include the fuel blocks (split between the graphite moderator and fuel contributions), CR 
blocks in Rings 1-4, side reflector blocks, permanent reflector blocks, and upper and lower reflectors. The 15 axial nodes ( 23 nodes in the 'no-sheath' model) in the heat structures are coupled with PHISICS in 12 kinetic zones (20 kinetic zones in the 'no-sheath' model), as the regions above and below the fuel are each grouped into one zone, along with the 10 (18 for 'no-sheath' model) fuel nodes.

The PHISICS/RELAP5-3D core calculation model is defined for a one-third core model shown in Figure A-12, where the core includes CR blocks, fuel blocks, and reflector blocks. The HTTR permanent reflector ring is one ring of non-hexagonal blocks around the side reflector blocks; however, for PHISICS modeling purposes, because the actual size is consistent with two prismatic blocks, it is modeled as two rings. Each of the kinetic zones is defined using a radial one-third mapping input that includes composition and temperature feedback.

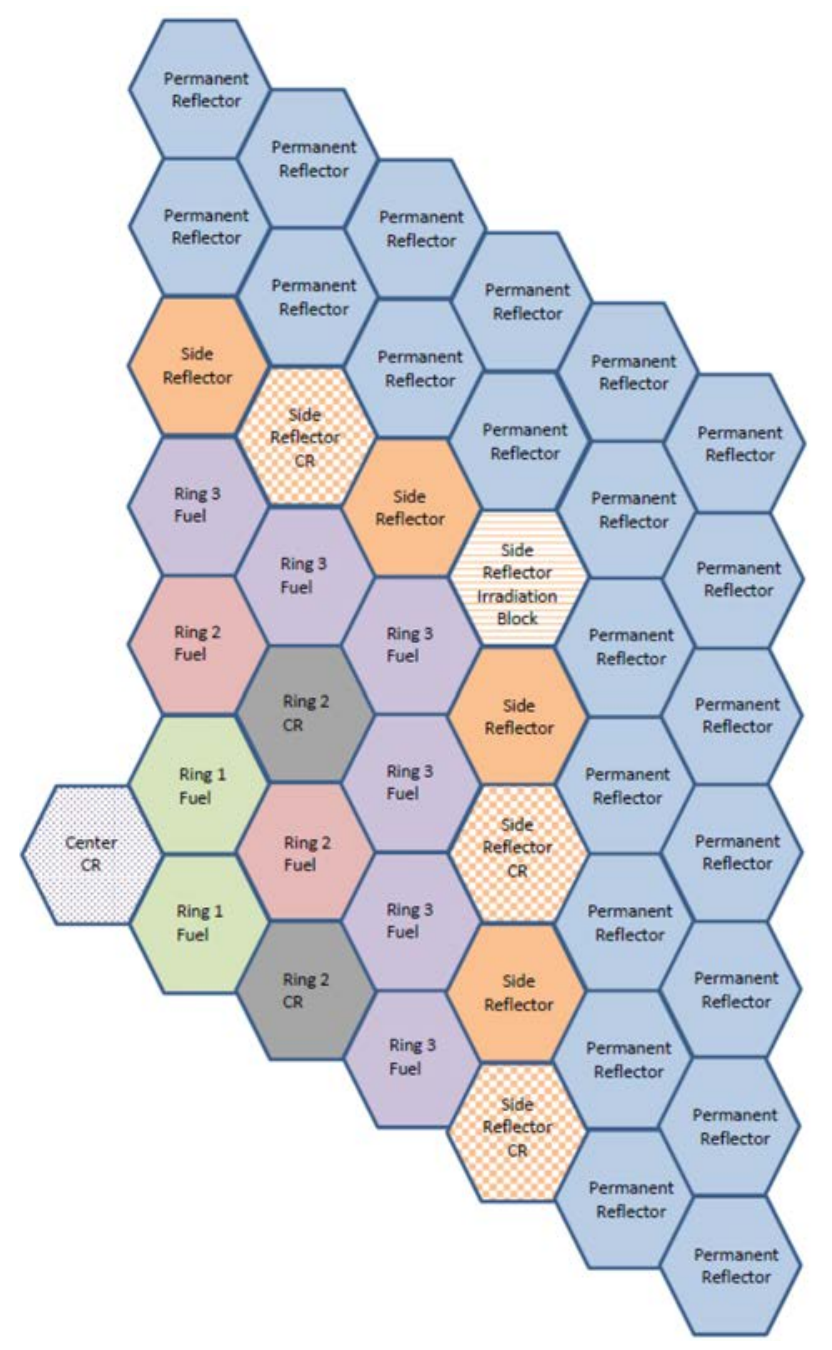

Figure A-12. PHISICS one-third core model.

The ability to model spatially-dependent temperature feedback with the cross-section look-up tables as well as the ability to utilize more than four energy groups (currently the limit in NESTLE) for a transport solution represent the two major advantages of using PHISICS to determine the flux distributions. The cross-section library was created using the methodology described in Section 4 (of the original document; not included with this excerpt). Temperature reactivity feedbacks are obtained by using the heat structure average temperatures as input to cross-section look-up tables to determine the cross-sections applicable for a specific state point. This process is repeated for every neutronics/thermal 
fluids iteration time step. The spatial power density data calculated by PHISICS are then passed back to RELAP5-3D in the next iteration as the new heat source data to be used as input to the fuel heat structures. This process continues for the duration of the defined steady state or transient run time.

\subsection{References}

1 Saito, S., et al., Design of the High Temperature Engineering Test Reactor, JAERI -1332, Japan Atomic Energy Research Institute, September 1994.

2 Fujimoto, N., et al., "Nuclear Design," Nuclear Engineering and Design, Vol. 233, pp. 23-36, 2004.

3 Iyoku, T., et al., "Design of Core Components," Nuclear Engineering and Design, Vol. 233, pp. 7179, 2004.

4 JAEA, "'Inherent Safety' in a High-Temp Gas-cooled Reactor Offer Self-protection," http://www.jaea.go.jp/jaeri/english/ff/news46/res.html, accessed October 2015.

5 Takamatsu, K., et al., "Spontaneous stabilization of HTGRs without reactor scram and core cooling Safety demonstration tests using the HTTR: Loss of reactivity control and core cooling," Nuclear Engineering and Design, Vol. 271, pp. 379-387, 2014.

6 OECD/NEA, "HTGR Reactor Performance during Loss-of-forced Cooling with HTTR," Organization for Economic Cooperation and Development /NEA Proposal HTTR-LOFC Project, 2009.

7 Kunitomi, K., et al., "Passive heat removal by vessel cooling system of HTTR during no forced cooling accidents," Nuclear Engineering and Design, Vol. 166, Issue 2, October 1996. 


\section{Appendix B}

\section{Implementation of an Adaptive Time Step Methodology for the PHISICS/RELAP5-3D Coupling Scheme}




\section{Appendix B}

\section{Implementation of an Adaptive Time Step Methodology for the PHISICS/RELAP5-3D Coupling Scheme}

\begin{tabular}{|l|l|}
\hline \multicolumn{2}{|l|}{$\begin{array}{l}\text { DIPARTIMENTO } \\
\text { DI INGEGNERAATRONAUTICA Implementation of an adaptive time } \\
\text { ELETTICAED ENERGETICA }\end{array}$} \\
\hline Step methodology for the PHISICS/ \\
RELAP5-3D ${ }^{\odot}$ coupling scheme
\end{tabular}

Appendix B 
Issue: 1 -December 23, 2016- First release

Issue: 2-March 22, 2017-Last release

\section{List of revisions}

\begin{tabular}{|l|l|l|}
\hline Issue & Date & Revision purpose \\
\hline 1 & December 23, 2016 & First release \\
\hline 2 & March 22, 2017 & Last release \\
\hline & & \\
\hline & & \\
\hline & & \\
\hline & & \\
\hline
\end{tabular}

INL_Contract_Technical_Report_MS1_v2.3_GS_RB.docx

Appendix B 


\section{Table of contents}

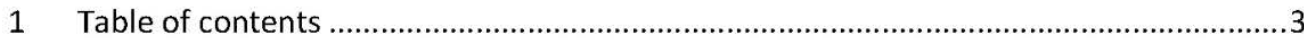

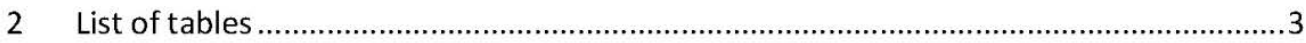

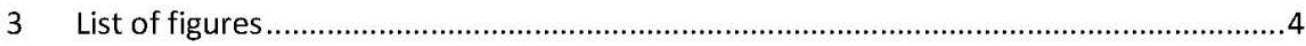

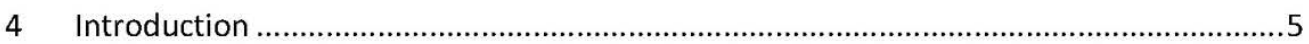

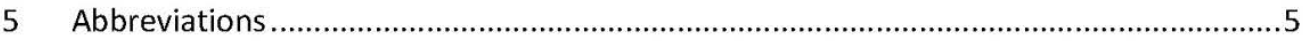

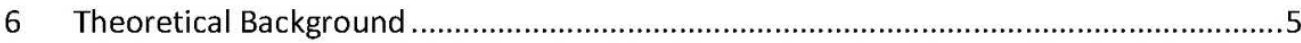

6.1 First order time discretization scheme of the odd parity version of the transport

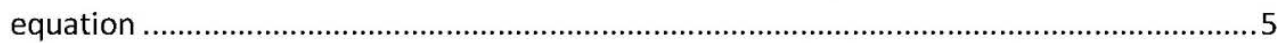

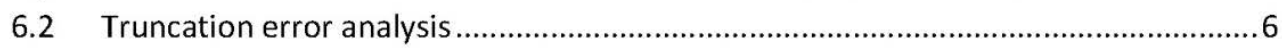

6.2.1 First order backward Euler scheme .......................................................

6.2.2 First order forward Euler scheme ................................................................ 7

6.2.3 First order central Euler scheme..................................................................... 8

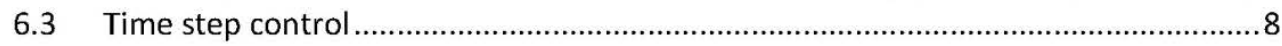

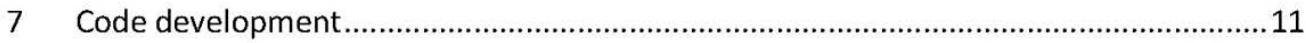

7.1 Source code description ................................................................................... 12

7.2 Time step control workflow................................................................................ 14

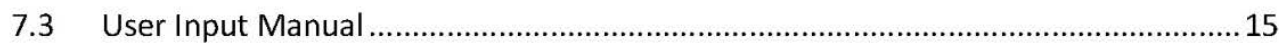

7.3.1 Time step decoupling new cards values ........................................................ 15

7.3.2 The TIME_STEP_CONTROL block .......................................................... 16

7.3.3 Time step control "_dtk.csv" file description .............................................20

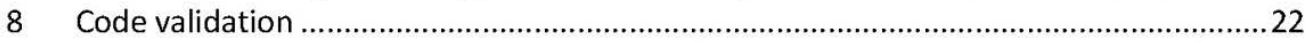

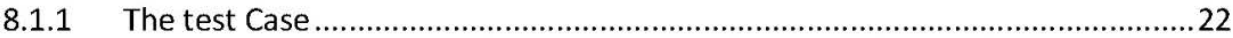

8.1.2 Analysis and results for all the methodologies ..............................................24

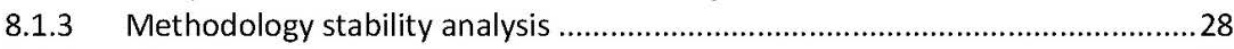

8.1.4 The time step smoothing ..................................................................... 33

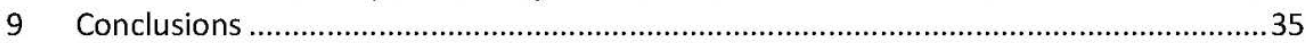

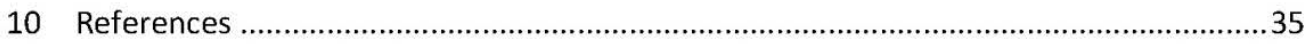

\section{List of tables}

Table 7.1 - Type and Functions for the adaptive time step calculation................................13

Table 7.2 - RELAP5-3D $\odot$ "Interface" subroutines................................................................. 14

Table 7.3 - "_dtk.csv" file structure ....................................................................................20

Table 7.4 - "_dtk.csv" variables description......................................................................22

Table 8.1- HTTR full power parameters .............................................................................2 23

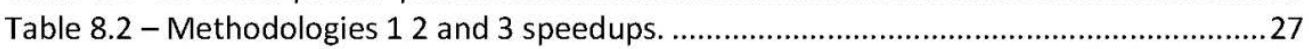

INL_Contract_Technical_Report_MS1_v2.3_GS_RB.docx

Page 3 of 36

Appendix B 
Table 8.3 - Methodologies 12 and 3 local relative tolerance on the flux speedups $(\tau=1 e-5)$.

Table 8.4- Methodologies 12 and 3 local absolute tolerance on the flux speedups $(\tau=1 \mathrm{e}-4)$.

Table 8.5 - Methodologies 1 local relative tolerance on the flux and smoothing speedups ( $\tau$ $=1 \mathrm{e}-5)$.

\section{List of figures}

Figure 7.1 - Adaptive time step function position and workflow .................................... 14

Figure 8.1 - Structure of the HTTR reactor ..................................................................23

Figure 8.2 - Loss of forced cooling test at $9 \mathrm{MW}$ Total Power ..........................................23

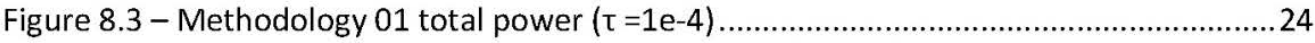

Figure 8.4 - Methodology 01 total power relative error $(\tau=1 \mathrm{e}-4)$....................................25

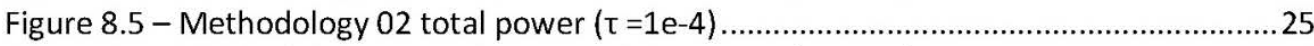

Figure 8.6 - Methodology 02 total power relative error $(\tau=1 \mathrm{e}-4)$....................................26

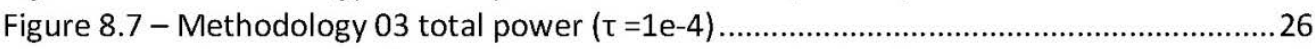

Figure 8.8 - Methodology 03 total power relative error $(\tau=1 \mathrm{e}-4)$...................................2 27

Figure 8.9 - Methodology $01,02,03$ total power with a relative tolerance of $\tau=1 \mathrm{e}-5 \ldots \ldots \ldots .28$

Figure 8.10 - Methodology 01-03 total power relative error with a relative tolerance of $\tau=1 \mathrm{e}-$

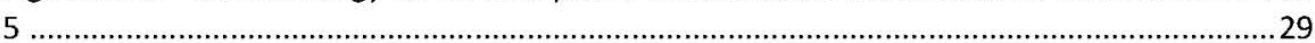

Figure 8.11 - Methodology $01,02,03$ neutronic time step, relative tolerance $(\tau=1 e-5)$......29

Figure 8.12 - Methodology $01,02,03$ flux second time derivative, relative tolerance $(\tau=1 e-5)$

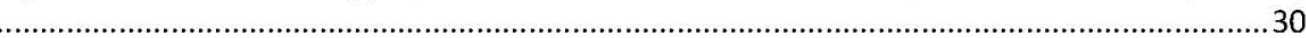

Figure 8.13 - Methodology $01,02,03$ total power, absolute tolerance $(\tau=1 \mathrm{e}-4) \ldots \ldots \ldots \ldots \ldots . . . . .31$

Figure 8.14 - Methodology $01,02,03$ total power relative error, absolute tolerance $(\tau=1 e-4)$

Figure 8.15 - Methodology $01,02,03$ neutronic time step, absolute tolerance $(\tau=1 \mathrm{e}-4)$.....32 Figure 8.16 - Methodology 01, 02,03 flux second time derivative, absolute tolerance ( $\tau=1 \mathrm{e}$ 4)

Figure 8.17 - Methodology 01 plus moving average and exponential smoothing total power $(\tau=1 \mathrm{e}-5)$ 33

Figure 8.18 - Methodology 01 plus moving average and exponential smoothing total power relative error $(\tau=1 \mathrm{e}-5)$ 34

Figure 8.19 - Methodology 01 moving average and exponential smoothing neutronic time $\operatorname{step}(\tau=1 \mathrm{e}-5)$

Figure 8.20 - Methodology 01 moving average and exponential smoothing flux second time derivative $(\tau=1 \mathrm{e}-5)$

INL_Contract_Technical_Report_MS1_v2.3_GS_RB.docx 


\section{Introduction}

The current work describes the implementation and validation of an adaptive time step methodology for the PHISICS/RELAP5-3D ${ }^{\odot}(P / R)$ coupling scheme. In Section 6 the theoretical basis of the adaptive time step are discussed; in section 7 the new code structure is explained and the new user input in the P/R input manual discussed; the results of the methodology validation with a simplified model of the HTTR are described in section 8. Finally, the conclusions from the test results analyses are presented in section 9.

\section{Abbreviations}

\begin{tabular}{|l|l|}
\hline Acronym & Description \\
\hline CR & Control Rod \\
\hline HTGR & High Temperature Gas Cooled Reactor \\
\hline HTTR & High Temperature Engineering Test Reactor \\
\hline INSTANT & $\begin{array}{l}\text { Intelligent Nodal and Semi-Structured Treatment for Advanced } \\
\text { Neutron Transport }\end{array}$ \\
\hline JAEA & Japan Atomic Energy Agency \\
\hline LOFC & Loss of Forced Cooling \\
\hline PHISICS & Parallel and Highly Innovative Simulation for INL code System \\
\hline RELAP & Reactor Excursion and Leak Analysis Program \\
\hline
\end{tabular}

\section{Theoretical Background}

\subsection{First order time discretization scheme of the odd parity version of the transport equation}

The PHISICS [1] transport core solver INSTANT [2] is based on the second order formulation of the transport equation discretized in angle by spherical harmonics while in space it uses orthonormal arbitrary order polynomials. To solve transient problems, a time dependent scheme has been implemented as a new module for the PHISICS suite. The chosen scheme is based on a first order backward Euler scheme with explicit delayed neutron treatment. In the following section a summary of its derivation is reported, while more details could be found in $[3,4,5]$. The time-dependent transport equation in the even-parity form is written in (1).

INL_Contract_Technical_Report_MS1_v2.3_GS_RB.docx 


$$
\left\{\begin{array}{l}
\frac{1}{u} \frac{\partial \psi}{\partial t}+\vec{\Omega} \cdot \vec{\nabla} \chi+\Sigma_{T} \psi=H^{+}[\psi]+S^{+}+\chi_{p}(1-\beta) P[\psi]+\sum_{i=1}^{N F} \chi_{i} \lambda_{i} C_{i} \\
\frac{1}{u} \frac{\partial \chi}{\partial t}+\vec{\Omega} \cdot \vec{\nabla} \psi+\Sigma_{T} \chi=H^{-}[\chi]+S^{-} \\
\frac{\partial C_{i}}{\partial t}=-\lambda_{i} C_{i}+\beta_{i} P[\psi] \quad i=1, \ldots, N F
\end{array}\right.
$$

In (1) the dependence of the variables on space, energy, direction and time have been suppressed; the boundary conditions and domain definitions have been omitted; and since an isotropic fission emission (prompt and delayed) is assumed it therefore appears only in the even equation. " $\psi$ " is the even component of the angular flux, " $\chi$ " is the odd component of the flux, " $H^{ \pm}[\mathrm{]}$ " is the even-odd scattering operator, " $P[\mathrm{]}$ " is the production operator, " $S^{ \pm "}$ is the even-odd external source. Applying a first order backward Euler scheme and solving explicitly for the delayed neutrons equations assuming constant cross sections and neutron delayed fraction during the time step (1) becomes:

$$
\left\{\begin{array}{l}
\frac{1}{u} \frac{\psi^{(n+1)}-\psi^{(n)}}{\Delta t_{n+1}}+\vec{\Omega} \cdot \vec{\nabla} \chi^{(n+1)}+\Sigma_{T}^{(n+1)} \psi^{(n+1)}=H^{+,(n+1)}\left[\psi^{(n+1)}\right]+S^{+,(n+1)} \\
+\chi_{p}^{(n+1)}\left(1-\beta^{(n+1)}\right) P^{(n+1)}\left[\psi^{(n+1)}\right]+\sum_{i=1}^{N F} \chi_{i}^{(n+1)} \lambda_{i} C_{i}^{(n+1)} \\
\frac{1}{u} \frac{\chi^{(n+1)}-\chi^{(n)}}{\Delta t_{n+1}}+\vec{\Omega} \cdot \vec{\nabla} \psi^{(n+1)}+\Sigma_{T}^{(n+1)} \chi^{(n+1)}=H^{-,(n+1)}\left[\chi^{(n+1)}\right]+S^{-,(n+1)} \\
C_{i}^{(n+1)}=C_{i}^{n} e^{-\lambda_{i} \Delta t_{n+1}}+\frac{1-e^{-\lambda_{i} \Delta t_{n+1}}}{\lambda_{i}} \beta^{(n)} P^{(n)}\left[\psi^{(n)}\right] \quad i=1, \ldots, N F
\end{array}\right.
$$

Equation (2) can be formally reduced to a "time independent" source problem that can be directly solved using INSTANT. Using a time discretization scheme at each iteration, an error with respect to the real solution is introduced and is called a truncation error. To assure solution convergence there are two ways: the first is to use a constant time step small enough to assure a small truncation error at each iteration during all the transient, the second is to use a variable time step that maintain the truncation error under a certain tolerance. The first approach is very robust but sometimes leads to a huge computational time waste, especially for transients in which very fast flux changes are followed by very slow changes, for example during a Control Rod (CR) ejection or Loss of Forced Cooling (LOFC) followed by a recriticality. The second approach is very attractive from the perspective of minimizing computational time, but the estimation of the truncation error depending on the time step size is needed to ensure solution convergence. In the next paragraphs the procedure for the time step size calculation will be presented.

\subsection{Truncation error analysis}

The truncation error specifies the approximation introduced using a finite summation of terms to approximate the exact time dependent solution which can be represented only by

INL_Contract_Technical_Report_MS1_v2.3_GS_RB.docx

Page 6 of 36 
the sum of an infinite series of terms. Using a Taylor series expansion of the solution, it is possible to see that the truncation error is related to the neglected terms in the Taylor series. Depending on the finite difference discretization scheme the truncation error assumes different forms as described in the next paragraphs. The space and energy dependence of the variables have been suppressed.

\subsubsection{First order backward Euler scheme}

The backward first order finite difference approximation of the flux first derivative is represented in (3).

$$
\left.\frac{\partial \bar{\phi}}{\partial t}\right|_{t=t_{n+1}}=\frac{\bar{\phi}^{(n+1)}-\bar{\phi}^{(n)}}{\Delta t_{n+1}}+\tau_{\bar{\phi}}^{(n+1)}
$$

Where: " $\bar{\phi}^{(n+1)}=\bar{\phi}\left(t_{n+1}\right)$ " is the scalar flux solution at the time " $t_{n+1}$ ", $\Delta t_{n+1}=t_{n+1}-t_{n}$ is the last time step and $\tau_{\phi}^{(n+1)}$ is the truncation error at the time " $t_{n+1}$ ". Considering the

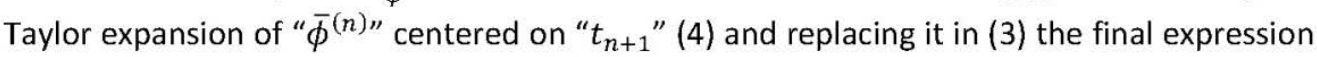
for the truncation error is obtained in (5).

$$
\begin{aligned}
& \bar{\phi}^{(n)}=\bar{\phi}^{(n+1)}-\left.\frac{\partial \bar{\phi}}{\partial t}\right|_{t=t_{n+1}} \Delta t_{n+1}+\left.\frac{\partial^{2} \bar{\phi}}{\partial t^{2}}\right|_{t=t_{n+1}} \frac{\Delta t_{n+1}^{2}}{2}+O\left(\Delta t_{n+1}\right)^{3} \\
& \tau_{\bar{\phi}}^{(n+1)}=\left.\frac{\partial^{2} \bar{\phi}}{\partial t^{2}}\right|_{t=t_{n+1}} \frac{\Delta t_{n+1}}{2}+O(\Delta t)^{2}
\end{aligned}
$$

where " $O(\Delta t)^{3 \prime}$ is the sum of the Taylor expansion terms of order greater than two (" $\Delta t_{n+1}$ " is assumed small enough so that the contribution of the terms with order greater than two is negligible).

\subsubsection{First order forward Euler scheme}

The forward first order finite difference approximation of the flux first derivative is represented in (6).

$$
\left.\frac{\partial \bar{\phi}}{\partial t}\right|_{t=t_{n}}=\frac{\bar{\phi}^{(n+1)}-\bar{\phi}^{(n)}}{\Delta t_{n+1}}+\tau_{\bar{\phi}}^{(n)}
$$

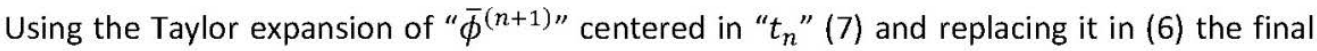
expression for the truncation error is obtained in (8).

$$
\begin{aligned}
& \bar{\phi}^{(n+1)}=\bar{\phi}^{(n)}-\left.\frac{\partial \bar{\phi}}{\partial t}\right|_{t=t_{n}} \Delta t_{n+1}+\left.\frac{\partial^{2} \bar{\phi}}{\partial t^{2}}\right|_{t=t_{n}} \frac{\Delta t_{n+1}^{2}}{2}+O\left(\Delta t_{n+1}\right)^{3} \\
& \tau_{\bar{\phi}}^{(n+1)}=-\left.\frac{\partial^{2} \bar{\phi}}{\partial t^{2}}\right|_{t=t_{n}} \frac{\Delta t_{n+1}}{2}+O(\Delta t)^{2}
\end{aligned}
$$

INL_Contract_Technical_Report_MS1_v2.3_GS_RB.docx 
Using a forward zero order Taylor expansion (" $\Delta t_{n+1}$ " is small enough to assume that the change of the second derivative " $O(\Delta t)$ " is negligible) it is possible to express the truncation error as a function of the second derivative calculated at " $t_{n+1}$ ":

$$
\tau_{\bar{\phi}}^{(n+1)}=-\left.\frac{\partial^{2} \bar{\phi}}{\partial t^{2}}\right|_{t=t_{n+1}} \frac{\Delta t_{n+1}}{2}+O(\Delta t)^{2}
$$

\subsubsection{First order central Euler scheme}

The forward first order finite difference approximation of the flux first derivative is represented in (6).

$$
\left.\frac{\partial \bar{\phi}}{\partial t}\right|_{t=t_{n}}=\frac{\bar{\phi}^{(n+1)}-\bar{\phi}^{(n-1)}}{\Delta t_{n}+\Delta t_{n+1}}+\tau_{\bar{\phi}}^{(n)}
$$

Using the Taylor expansion of " $\bar{\phi}^{(n+1) \text { " }}$ and " $\bar{\phi}^{(n-1) \text { " }}(11),(12)$ centered on " $t_{n}$ " and replacing them in (10) the final expression for the truncation error is obtained in (13).

$$
\begin{aligned}
& \bar{\phi}^{(n+1)}=\phi^{(n)}+\left.\frac{\partial \bar{\phi}}{\partial t}\right|_{t=t_{n}} \Delta t_{n+1}+\left.\frac{\partial^{2} \bar{\phi}}{\partial t^{2}}\right|_{t=t_{n}} \frac{\Delta t_{n+1}{ }^{2}}{2}+O(\Delta t)^{3} \\
& \phi^{(n-1)}=\phi^{(n)}-\left.\frac{\partial \phi}{\partial t}\right|_{t=t_{n}} \Delta t_{n}+\left.\frac{\partial^{2} \bar{\phi}}{\partial t^{2}}\right|_{t=t_{n}} \frac{\Delta t_{n}{ }^{2}}{2}+O(\Delta t)^{3} \\
& \tau_{\bar{\phi}}^{(n+1)}=-\left.\frac{\partial^{2} \bar{\phi}}{\partial t^{2}}\right|_{t=t_{n+1}} \frac{\Delta t_{n+1}}{2}+O(\Delta t)^{2}
\end{aligned}
$$

Using a forward zero order Taylor expansion in this case it is also possible to express the truncation error as a function of the second derivative calculated at " $t_{n+1}$ ":

$$
\tau_{\bar{\phi}}^{(n+1)}=-\left.\frac{\partial^{2} \bar{\phi}}{\partial t^{2}}\right|_{t=t_{n+1}} \frac{\left(\Delta t_{n+1}-\Delta t_{n}\right)}{2}+O(\Delta t)^{2}
$$

Equation (14) shows that the central discretization scheme is equivalent to the second order backward Euler scheme only when the time step is constant.

\subsection{Time step control}

As described in paragraph 6.1 the time evolution scheme implemented in PHISICS is a first order backward Euler scheme, therefore the truncation error has the form described in (5). Using (5) it is possible to calculate the truncation error only at time " $t_{n+1}$ ". Using a forward zero order Taylor expansion (" $\Delta t_{n+2}$ " is small enough to assume that the change of the second derivative " $O(\Delta t)$ " is negligible) the truncation error of the next iteration " $t_{n+2}$ " can be calculated as function of the next time step " $\Delta t_{n+2}$ ":

INL_Contract_Technical_Report_MS1_v2.3_GS_RB.docx 


$$
\begin{aligned}
& \tau_{\bar{\phi}}^{(n+2)}=\left(-\left.\frac{\partial^{2} \bar{\phi}}{\partial t^{2}}\right|_{t=t_{n+1}}+O(\Delta t)\right) \frac{\Delta t_{n+2}}{2}+O(\Delta t)^{2} \\
& =-\left.\frac{\partial^{2} \bar{\phi}}{\partial t^{2}}\right|_{t=t_{n+1}} \frac{\Delta t_{n+2}}{2}+O(\Delta t)^{2}
\end{aligned}
$$

Using the (13) and defining an absolute tolerance on the truncation error "tol ${ }_{a b s}$ ", the corresponding maximum time step that ensures a truncation error smaller than the tolerance can be calculated:

$$
\operatorname{tol}_{a b s} \geq\left.\left.\left|\frac{\partial^{2} \bar{\phi}}{\partial t^{2}}\right|_{t=t_{n+1}}\left|\frac{\Delta t_{n+2}}{2} \Rightarrow \Delta t_{n+2} \leq 2 t o l_{a b s}\right| \frac{\partial^{2} \bar{\phi}}{\partial t^{2}}\right|_{t=t_{n+1}}\right|^{-1}
$$

The use of an absolute tolerance may be not appropriate if the flux change by orders of magnitude during the transient. In these cases, it is better to define a relative tolerance " $t o l_{\text {rel }}$ " on the time integrated truncation error:

$$
\operatorname{tol}_{r e l} \bar{\phi}^{(n+1)} \geq\left|\frac{\partial^{2} \bar{\phi}}{\partial t^{2}}\right|_{t=t_{n+1}} \mid \frac{\Delta t_{n+2}^{2}}{2} \Rightarrow \Delta t_{n+2} \leq \sqrt{\left.2 t o l_{r e l} \bar{\phi}^{(n+1)}\left|\frac{\partial^{2} \bar{\phi}}{\partial t^{2}}\right|_{t=t_{n+1}}\right|^{-1}}
$$

To estimate the new time step using (16) and (17) it is necessary to calculate the second time derivative of the flux. The easiest way to calculate the second derivative at the time " $t_{n+1}$ " is to use the standard three-point estimate of the second derivative:

$$
\left.\frac{\partial^{2} \bar{\phi}}{\partial t^{2}}\right|_{t=t_{n+1}} \approx 2 \frac{\Delta t_{n} \bar{\phi}^{(n+1)}-\left(\Delta t_{n}+\Delta t_{n+1}\right) \bar{\phi}^{(n)}+\Delta t_{n+1} \bar{\phi}^{(n-1)}}{\Delta t_{n} \Delta t_{n+1}\left(\Delta t_{n}+\Delta t_{n+1}\right)}
$$

Considering the different forms assumed by the truncation error depending on the implemented scheme it is possible to estimate the second derivative using a combination of them. The truncation error described above is for the definition of an error on the first derivative of the flux. The error introduced in the flux solution by the truncation error with respect to the reference solution can be calculated integrating (5), (9) and (14) respectively for the backward central and forward schemes:

$$
\begin{aligned}
& \bar{\phi}_{e x}^{(n+1)}-\bar{\phi}_{1 b}^{(n+1)} \approx-\left.\frac{\partial^{2} \bar{\phi}}{\partial t^{2}}\right|_{t=t_{n+1}} \frac{\Delta t_{n+1}{ }^{2}}{2} \\
& \bar{\phi}_{e x}^{(n+1)}-\bar{\phi}_{1 f}^{(n+1)} \approx+\left.\frac{\partial^{2} \bar{\phi}}{\partial t^{2}}\right|_{t=t_{n+1}} \frac{\Delta t_{n+1}{ }^{2}}{2}
\end{aligned}
$$




$$
\bar{\phi}_{e x}^{(n+1)}-\bar{\phi}_{1 c}^{(n+1)}=\left.\frac{\partial^{2} \bar{\phi}}{\partial t^{2}}\right|_{t=t_{n+1}} \frac{\left(\Delta t_{n+1}{ }^{2}-\Delta t_{n}{ }^{2}\right)}{2}
$$

Subtracting (20) from (19) and (21) from (19), two new expressions for the second derivative estimation are obtained:

$$
\begin{aligned}
& \left.\frac{\partial^{2} \bar{\phi}}{\partial t^{2}}\right|_{t=t_{n+1}} \approx \frac{\bar{\phi}_{1 b}^{(n+1)}-\bar{\phi}_{1 f}^{(n+1)}}{\Delta t_{n+1}{ }^{2}} \\
& \left.\frac{\partial^{2} \bar{\phi}}{\partial t^{2}}\right|_{t=t_{n+1}} \approx 2 \frac{\bar{\phi}_{1 b}^{(n+1)}-\bar{\phi}_{1 c}^{(n+1)}}{2 \Delta t_{n+1}{ }^{2}-\Delta t_{n}{ }^{2}}
\end{aligned}
$$

The time discretion scheme implemented in PHISICS is the first order backward Euler scheme,

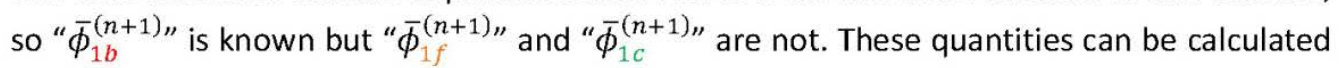
using the following expressions:

$$
\begin{aligned}
& \frac{1}{v} \frac{\bar{\phi}_{1 c}^{(n+1)}-\bar{\phi}_{1 b}^{(n-1)}}{\Delta t_{n+1}+\Delta t_{n}}=S_{n}^{+}-\Sigma_{R}{ }^{(n)} \bar{\phi}_{1 b}^{(n)}-\frac{1}{V} \sum_{\alpha=1}^{N s u r f_{r}} \int_{S_{\alpha}} d s_{\alpha}^{i} \vec{J} \cdot \vec{n} \\
& \frac{1}{v} \frac{\bar{\phi}_{1 f}^{(n+1)}-\bar{\phi}_{1 b}^{(n)}}{\Delta t_{n}}=S_{n}^{+}-\Sigma_{R}{ }^{(n)} \bar{\phi}_{1 b}^{(n)}-\frac{1}{V} \sum_{\alpha=1}^{N s u r f_{r}} \int_{S_{\alpha}} d s \vec{J}_{\alpha}^{(n)} \cdot \vec{n}
\end{aligned}
$$

Here " $\mathcal{J}_{\alpha}^{(n)}$ " is the net current on the surface " $S_{\alpha}$ " and $\Sigma_{R}{ }^{(n)}$ is the removal cross section. The quantity on the right side of (24) and (25) is available from the use of the backward scheme at the time " $t_{n}$ ":

$$
\frac{1}{v} \frac{\bar{\phi}_{1 b}^{(n)}-\bar{\phi}_{1 b}^{(n-1)}}{\Delta t_{n}}=S_{n}^{+}-\Sigma_{R}{ }^{(n)} \bar{\phi}_{1 b}^{(n)}-\frac{1}{V} \sum_{\alpha=1}^{N s u r f_{r}} \int_{S_{\alpha}} d s \vec{J}_{\alpha}^{(n)} \cdot \vec{n}
$$

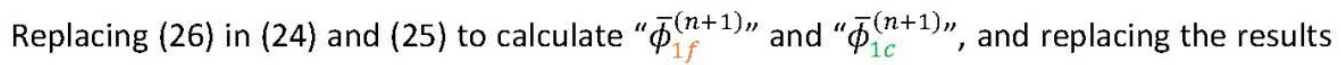
in (22) and (23), two alternative estimations of the second derivative are obtained:

$$
\begin{aligned}
& \left.\frac{\partial^{2} \phi}{\partial t^{2}}\right|_{t=t_{n+1}} \approx \frac{\left(\phi_{1 b}^{(n+1)} \Delta t_{n}-\bar{\phi}_{1 b}^{(n)}\left(\Delta t_{n+1}+\Delta t_{n}\right)+\bar{\phi}_{1 b}^{(n-1)} \Delta t_{n+1}\right)}{\Delta t_{n+1}{ }^{2} \Delta t_{n}} \\
& \left.\frac{\partial^{2} \bar{\phi}}{\partial t^{2}}\right|_{t=t_{n+1}} \approx 2 \frac{\left(\bar{\phi}_{1 b}^{(n+1)} \Delta t_{n}-\bar{\phi}_{1 b}^{(n)}\left(\Delta t_{n+1}+\Delta t_{n}\right)+\bar{\phi}_{1 b}^{(n-1)} \Delta t_{n+1}\right)}{\Delta t_{n}\left(2 \Delta t_{n+1}{ }^{2}-\Delta t_{n}{ }^{2}\right)}
\end{aligned}
$$


Equations (18), (27) and (28) differ only in the denominator and if the time step is the same they have the same value. The last two expressions should ensure a more stable time step calculation when the operators' time dependence of (2) is small, as demonstrated in [6].

In the previous paragraphs the space and energy dependencies have been suppressed, but using (16) and (17) a time step can be calculated for each subdomain and energy group. The minimum calculated time step corresponds to the maximum predicted error therefore selecting it for the next iteration it ensures that the truncation error for each group and spatial subdomain is smaller than the tolerance:

$$
\begin{aligned}
\Delta t_{n+2} & =\min _{1<s<N_{s}} \min _{1<g<N_{g}}\left(\left.2 t o l_{a b s}\left|\frac{\partial_{g}^{2} \bar{\phi}}{\partial t^{2}}\right|_{t=t_{n+1}}\right|^{-1}\right) \\
\Delta t_{n+2} & =\min _{1<s<N_{s}} \min _{1<g<N_{g}}\left(\sqrt{\left.2 t o l_{\text {rel }}^{s} \bar{\phi}^{(n+1)}\left|\frac{\partial_{g}^{2} \bar{\phi}}{\partial t^{2}}\right|_{t=t_{n+1}}\right|^{-1}}\right)
\end{aligned}
$$

Where " ${ }_{g} \bar{\phi}$ " is the group " $g$ " scalar flux in the subdomain " $s$ ", " $N_{s}$ " is the total number of subdomain and " $N_{g}$ " is the total number of groups. In some cases, the selected group and subdomain for the time step calculation changes very fast introducing instability in the calculations, therefore it is sometimes preferable to use an average flux instead of the local one:

$$
\begin{aligned}
& \Phi_{s}=\sum_{g=1}^{N_{g}} \bar{g} \\
& \Phi_{g}=\frac{\sum_{S=1}^{N_{s}}{ }_{g}^{s} \bar{\phi} V_{S}}{\sum_{s=1}^{N_{S}} V_{S}} \\
& \Phi=\frac{\sum_{S=1}^{N_{S}} \sum_{g=1}^{N_{g}}{ }_{g}^{s} \bar{\phi} V_{s}}{\sum_{S=1}^{N_{S}} V_{S}}
\end{aligned}
$$

Where " $\Phi_{s}$ " is the total scalar flux in the subdomain " $s$ ", " $\Phi_{g}$ " is the group " $g$ " average scalar flux, " $\Phi$ " is the total average scalar flux and " $V_{S}$ " is the subdomain volume.

\section{Code development}

The current RELAP5-3D ${ }^{\odot}$ adaptive time step methodology is based on the calculation of the time step using the time first derivative of the scalar flux and the cross-sections. Three new approaches based on the flux second derivative estimation were presented in the previous paragraph. Each methodology can be used with "s ${ }_{g} \bar{\phi} ", \Phi_{s}, \Phi_{g}$ or $\Phi$ and an option to use the subdomain fission power " $P$ " or the total power " $P_{f}$ " has also been included. The six different variables can be used with each of the three methodologies in combination with an

INL_Contract_Technical_Report_MS1_v2.3_GS_RB.docx

Page 11 of 36

\section{Appendix B}


absolute or relative tolerance; therefore, 36 different options are globally available to calculate the new time step. The possibility of using a combination of them (the minimum calculated time step will be selected) is available for instance when an absolute tolerance is used until the predicted time step became too large, after which a relative tolerance is used to maintain the time step at a reasonable size, even when the flux decreases by several orders of magnitude.

\subsection{Source code description}

To implement the adaptive time step in the P/R coupled code several subroutines have been developed and included in the "time integrator" module of PHISICS. Six main subroutines and one new derived data type for a total of more than 2,000 lines of code have been added. Some modifications to the original RELAP5-3D ${ }^{\odot}$ code and the interface has been also added. A summarized description of the types and the subroutines implemented in the code is presented in Table 7.1.

\begin{tabular}{|c|c|}
\hline NAME & DESCRIPTION \\
\hline ts_cntrl (TYPE) & $\begin{array}{l}\text { It is the new derived data type for the time step decoupling } \\
\text { control. The pointer "TS_CNTRL" included in the } \\
\text { "Instant_inter_mode.F" module (the module in which all the } \\
\text { interface functions are stored) has this type and is shared by all } \\
\text { the newly-developed functions. This type stores all the options } \\
\text { included in the XML input file described in paragraph } 7.3 \text { and all } \\
\text { the data required by the solvers to calculate the time step, such } \\
\text { as the flux solutions of the last three iterations, subdomain } \\
\text { volumes, power etc.. }\end{array}$ \\
\hline Init_ts_cntrl & $\begin{array}{l}\text { This subroutine allocates and initializes the TS_CNTRL branches, } \\
\text { e.g. the first flux solution taken from the transient restart file. }\end{array}$ \\
\hline Comp_ts_cntrl & $\begin{array}{l}\text { This subroutine calculates the new time step following the } \\
\text { workflow described in paragraph } 6 \text {. The theory is implemented } \\
\text { as described above but some additional features are included: } \\
\text { - Exponential moving average: The estimation of " } \Delta t_{n+2} \text { " } \\
\text { cannot be classified as linear partial implicit. For this } \\
\text { reason, the estimation of this quantity could be affected } \\
\text { by instabilities. To reduce this effect an exponential } \\
\text { smoothing and a moving average is included in the } \\
\text { subroutine and available as an option. } \\
\text { - Rationalization: When the Thermal Hydraulic (TH) and } \\
\text { the Neutron Kinetic (NK) has two different time steps } \\
\text { one of them sub-cycles the other. If the bigger time step } \\
\text { is not a rational multiple of the other, the two solvers do } \\
\text { not synchronize. The subroutine approximates the new }\end{array}$ \\
\hline
\end{tabular}

INL_Contract_Technical_Report_MS1_v2.3_GS_RB.docx

Page 12 of 36

Appendix B 


\begin{tabular}{|c|c|}
\hline & $\begin{array}{l}\text { NK time step as the maximum rational multiple or divisor } \\
\text { of the TH time step, instead of the calculated value. } \\
\text { Max, Min thresholds: It is possible to define a maximum } \\
\text { and minimum threshold for the time step. This is very } \\
\text { useful especially when an absolute tolerance is used to } \\
\text { avoid that the predicted time step becomes too small or } \\
\text { too big. } \\
\text { - Constant time step: if the maximum and the minimum } \\
\text { time step are equal, this value is used instead of the } \\
\text { calculated time step. }\end{array}$ \\
\hline Read_ts_cntrl_from_xml & $\begin{array}{l}\text { This subroutine reads all parameters in the } \\
\text { "TIME_STEP_CONTROL" block of the XML input file for the time } \\
\text { step decoupling. }\end{array}$ \\
\hline Print_ts_cntrl & $\begin{array}{l}\text { This subroutine prints all the results of the new time step } \\
\text { calculation; i.e. the flux second time derivative, the non- } \\
\text { rationalized time step, the selected energy group and } \\
\text { subdomain etc... The results are printed in a csv file with suffix } \\
\text { "-dtk.csv" described in the paragraph } 7.3 \text {. The printing of the } \\
\text { data is not done at every iteration, but a buffer is included that } \\
\text { stores the data and prints the information every } 10 \text { iterations. }\end{array}$ \\
\hline Destructor_ts_cntrl & $\begin{array}{l}\text { This subroutine deallocates all the variables used in the time } \\
\text { step methodology at the end of the transient including the } \\
\text { "TS_CTRL" pointer. }\end{array}$ \\
\hline Local_error_ts_cntrl & $\begin{array}{l}\text { This function is the local error handler that stops the calculation } \\
\text { and writes an error message in the output file with a description } \\
\text { of the error, the code number and depending on the error a } \\
\text { relevant integer, real or string. }\end{array}$ \\
\hline
\end{tabular}

Table 7.1 - Type and Functions for the adaptive time step calculation.

The subroutines "Init_ts_cntrl" and "Comp_ts_cntrl" are parallelized to collect the data from all the CPUs, while the other subroutines are executed only by the main process (CPU0). These subroutines have been developed as an independent module that can be called by PHISICS for many different purposes, i.e. standalone time evolution. To make these subroutines specific for the P/R time evolution scheme instead of being directly called in RELAP5-3D ${ }^{\odot}$, "interface" subroutines have been added to the interface file "Instant_inter_mode.F". The names of these subroutines is formed by the combination of the original PHISICS subroutine and the RELAP5-3D ${ }^{\odot}$ subroutine that called it.

\begin{tabular}{|l|l|}
\hline \multicolumn{1}{|c|}{ NAME } & \multicolumn{1}{c|}{ DESCRIPTION } \\
\hline Read_ts_cntrl_from_xml_rdtkin & $\begin{array}{l}\text { This subroutine calls "Read_ts_cntrl_from_xml" in } \\
\text { "rdtkin". }\end{array}$ \\
\hline Comp_ts_cntrl_tran & This subroutine calls "Comp_ts_cntrl" in "tran". \\
\hline Print_ts_cntrl_tran(opt) & $\begin{array}{l}\text { This subroutine calls "Print_ts_cntrl" in "tran". It has an } \\
\text { argument "opt" because it is called twice in "tran": first }\end{array}$ \\
\hline
\end{tabular}

INL_Contract_Technical_Report_MS1_V2.3_GS_RB.docx

Page 13 of 36 


\begin{tabular}{|l|l|}
\hline & $\begin{array}{l}\text { (opt=1) during normal calculation secondly (opt=2) after } \\
\text { the last iteration to print the remaining data in the } \\
\text { buffer. }\end{array}$ \\
\hline Open_dtk_csv_file_idtkin & $\begin{array}{l}\text { This subroutine is called by "rdtkin" but does not call a } \\
\text { PHISICS subroutine. It just opens the "_dtk.csv" output } \\
\text { file. }\end{array}$ \\
\hline Init_ts_cntrl_instant_tr & This subroutine calls "Init_ts_cntrl" in "instant_tr". \\
\hline
\end{tabular}

Table 7.2 - RELAP5-3D "Interface" subroutines.

The "Destructor_ts_cntrl" and "Local_error_ts_cntrl" are accessory subroutines so they are called directly by RELAP5-3D ${ }^{\odot}$ without an "interface". In the paragraph below the workflow of the time step decoupling is described.

\subsection{Time step control workflow}

In Figure 7.1 the workflow of the RELAP5-3D ${ }^{\odot}$ section that use the adaptive time step subroutines is represented. At the beginning, before the problem initialization, the options are read from the XML input file and, if needed, the output file is opened (steps 1, 2). After that, the first call of the print and calculation functions (steps 3,4) are skipped because the solution is not yet initialized. At this point the solution is initialized (step 5) and the first real call of the printing function is done (step 6). The printing and calculation subroutines are called alternatively to give the new time step to the NK solver and to print the new iteration information until the end of the transient.

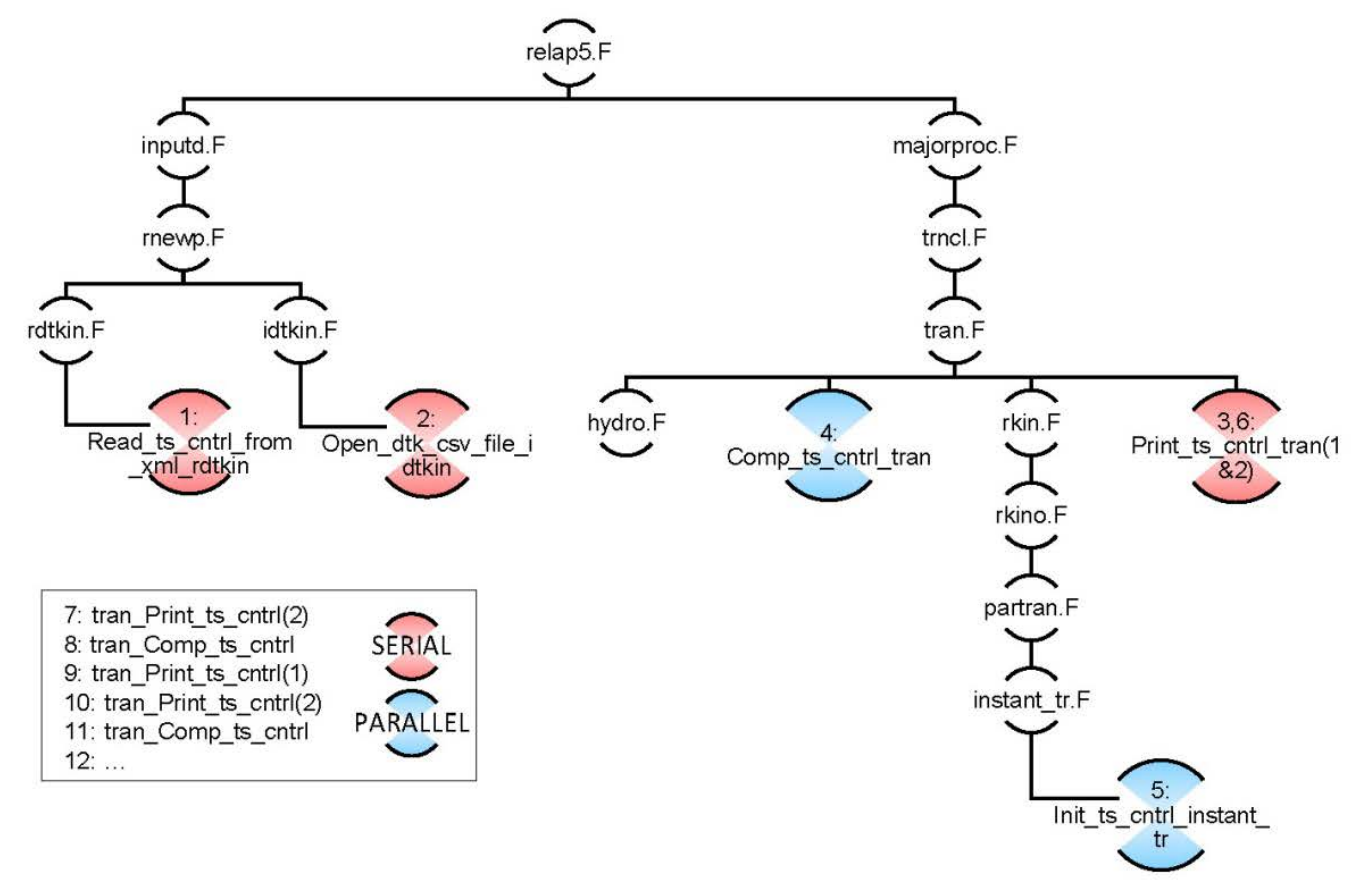

Figure 7.1 - Adaptive time step function position and workflow

Page 14 of 36

INL_Contract_Technical_Report_MS1_v2.3_GS_RB.docx

Appendix B 
In Figure 7.1 it is possible to see that the adaptive time step control logic is mainly included in the "tran.F" subroutine, where the "hydro.F" function performs the TH advancement and the "rkin. F" instead performs the NK advancement. The logic in "tran.F" uses the TH and NK time step sizes $\left(\Delta t_{T H} \geq\right.$ or $\left.<\Delta t_{N K}\right)$ to determine if the "hydro.F" and the "rkin.F" subroutines are called or skipped.

\subsection{User Input Manual}

In the current paragraph the new section of the P/R user input manual is reported.

\subsubsection{Time step decoupling new cards values}

The kernel used for the TH and NK decoupling is the same that RELAP5-3D ${ }^{\odot}$ uses for NESTLE, therefore, almost all the cards used in RELAP5-3D ${ }^{(\odot)}$ have the same value as described in [7].

\section{Card 2200, Kinetics Time Step Control}

Wuiflf Kinetics time step control option: this word is used as described in [7]. If 0 the $\leq T I M E$ STEP CONTROL $>$ block in the inst_cont.xml file it is not read and the target maximum kinetics time step (W3 of card 2201-2299) will be used.

W2(I) Kinetics time step format: applied as described in [7]. Only the absolute time step size option $(\mathrm{W} 2=0)$ has been tested.

W3(I) Trip unit for locking time steps: applied as described in [7]. Only the default option 0 has been tested.

W4(I) Trip unit for unlocking time steps: applied as described in [7]. Only the default option 0 has been tested.

W5(I) Control variable unit number: applied as described in [7]. Only the default option 0 has been tested.

W6(I) Flux extrapolation option: applied as described in [7]. The flux extrapolation option is not yet active. W6=1 is allowed but no flux extrapolation will be performed.

W7(I) This word specifies a new option. The new option 2 allow the user to print a "_dtk.csv" file (the file name is composed by the R5-3D output file name and the "_dtk" suffix with a ".csv" extension) in which all the information about the new NK time step estimation will be printed. This file will be described in the paragraph 7.3.3. Option 1 is for printing the typical RELAP5-3D ".dtk" file described in [7]. The value of this card is overridden by the <echo> element in the inst_cont.xml file, as described in paragraph 7.3.2.

\section{Cards 2201 through 2299, Kinetics Time Step Data}

The cards are used as described in [7], except when the maximum and the minimum time step are equal. When the maximum and the minimum time step are equal a constant time step is used.

INL_Contract_Technical_Report_MS1_v2.3_GS_RB.docx

Page 15 of 36

Appendix B 
W2(R) Target minimum kinetics time step (s): used as described in [7]. Only the absolute time step size option has been tested. Although it is possible to specify a NK time step smaller than the TH one, until now, only a bigger NK time step has been tested.

W3(R)

Target maximum kinetics time step: used as described in [7]. Only the absolute time step size option has been tested. Although it is possible to specify a NK time step smaller than the TH time step, only a bigger NK time step has so far been tested.

Example:

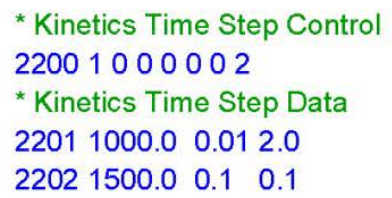

The previous example allows the NK time step to vary between $0.01 \mathrm{~s}$ and $2.0 \mathrm{~s}$ for the first $1000 \mathrm{~s}$ simulation, for the next 500 s the constant $0.1 \mathrm{~s}$ time step will be used. The ".csv" file will be printed during the transient.

\subsubsection{The TIME_STEP_CONTROL block}

The $\angle T I M E$ STEP_CONTROL $>$ block contains all the information required by the code to calculate the NK time step if W1 of card 2200 is 1 . The three methodologies for calculating the new NK time step were implemented based on equations $[4,8,6]$.

- <debug_out>

Type: integer

Default: 0

Description: 1 to switch on the debug printing. Values other than 0 should be used with care since they produce voluminous output and can easily make extremely large output files.

- <echo>

Type: integer

Default: if not specified the R5-3D value will be used (W7 card 2200)

Description: 1 to print normal ".dtk" file output as described in [7], 2 to print the new .csv file described in paragraph 7.3.3.

- <echo_skip>

Type: integer

Default: -1

Description: this element is needed only when <echo> is 2. A value of $\mathrm{N}$ allows to skip $\mathrm{N}-1$ printing in the new ". $\mathrm{csv}$ " file (if $\mathrm{N}=1$ no skip at all). A value of -1 allows printing only when $\mathrm{TH}$ and NK are in sync.

INL_Contract_Technical_Report_MS1_v2.3_GS_RB.docx

\section{Appendix B}


- <exp_smoot>

Type: real

Default: -1

Description: if $>0$ the exponential smoothing will be applied to the time step calculation. The value of this element is the smoothing factor $\alpha$, and can vary between 0 and 1 .

$$
\Delta t^{(n+2)}=\Delta t_{n+1}(1-\alpha)+\alpha \Delta t^{p}
$$

A value of -1 deactivates the time step filtering. When $<m a \_s m o o t>$ is not -1 the only allowed value is -1 .

- <ma_smoot>

Type: integer

Default: -1

Description: if $>1$ a $\mathrm{N}_{\mathrm{MA}}$ degree moving average will be applied to the time step calculation. The value of this element is the moving average degree.

$$
\Delta t^{(n+2)}=\frac{\sum_{i=1}^{N_{M A}-1} \Delta t^{(n+2-i)}+\Delta t^{p}}{N_{M A}}
$$

A value of -1 deactivates the time step filtering. When <exp_smoot $>$ is not -1 , the only allowed value is -1 .

- <min_max>

Type: string

Default: $\min$

Description: when multiple methodologies or tolerances are used, if min the minimum time step is selected and if max the maximum time step is selected. When only one methodology or tolerance is used this card is not needed.

- <Methodology_01>

Description: This block controls the input requirements for the first methodology. The first methodology is based on the truncation error estimation using the second derivative of the flux/power. To estimate the second derivative a simple backward scheme is used:

$$
\left.\frac{\partial^{2} \phi}{\partial t^{2}}\right|_{t=t_{n+1}}=2 \frac{\Delta t_{n} \phi^{(n+1)}-\left(\Delta t_{n}+\Delta t_{n+1}\right) \phi^{(n)}+\Delta t_{n+1} \phi^{(n-1)}}{\Delta t_{n} \Delta t_{n+1}\left(\Delta t_{n}+\Delta t_{n+1}\right)}
$$

- [Is_on]

Type: attribute logical

Default: " $\mathrm{t}$ ". This attribute is optional, if not specified the default values is used.

Description: if " $\mathrm{t}$ " the methodology will be used otherwise it is off. If it is activated at least one of the tolerances must be defined and not equal to -1 .

- <flux_toll>

Type: real

Default: -1

Description: if $>0$ the time step will be calculated to maintain the integrated truncation error on the flux smaller than the tolerance " $\epsilon$ ".

$$
\Delta t^{(n+2)}=\sqrt{2 \epsilon \phi^{(n+1)}\left|\frac{\partial^{2} \phi^{(n+1)}}{\partial t^{2}}\right|^{-1}}
$$

INL_Contract_Technical_Report_MS1_v2.3_GS_RB.docx

\section{Appendix B}


Four different tolerance types are allowed.

- [type]

Type: attribute string

Default: "loc", this attribute is optional if not inputted the default values is used.

Description: "loc" the time step is calculated in each subdomain/energy group and the smallest one is selected to maintain the error below the tolerance in each

subdomain/energy group; "grp" the flux is collapsed to one energy group than the time step is calculated in each subdomain and the smallest one is selected; "sbd" the flux is volume averaged than the time step is calculated in each group and the smallest one is selected. "tot" the flux is collapsed to one group and then volume averaged finally the time step is calculated.

- <flux1_toll>

Type: real

Default: -1

Description: if $>0$ the time step will be calculated to maintain the truncation error on the flux smaller than the tolerance " $\tau$ ".

$$
\Delta t^{(n+2)}=2 \tau\left|\frac{\partial^{2} \phi^{(n+1)}}{\partial t^{2}}\right|^{-1}
$$

The tolerance is normalized with the average flux of the first iteration; therefore the tolerance must be specified as a relative value (e.g. 1e-1, 1e-3, 1e-4...) of the initial average flux. Four different tolerance types are allowed.

- [type]

Type: attribute string

Default: "loc"

Description:

- If "loc": the time step is calculated in each subdomain/energy group and the smallest value is selected to maintain the error below the tolerance for all the subdomains/energy groups.

- If "grp": the flux is collapsed to one energy group, the time step is calculated in each subdomain and the smallest value is selected.

- If "sbd": the flux is volume-averaged, the time step is calculated in each group and the smallest value is selected.

- If "tot": the flux is collapsed to one group and volume-averaged and finally the time step is calculated.

- <pow_toll>

Type: real

Default: -1

Description: if $>0$ the time step will be calculated to maintain the integrated truncation error on the power smaller than the tolerance " $\epsilon$ ".

_Contract_Technical_Report_MS1_v2.3_GS_RB.docx

\section{Appendix B}




$$
\Delta t^{(n+2)}=\sqrt{2 \epsilon \phi^{(n)}\left|\frac{\partial^{2} P^{(n+1)}}{\partial t^{2}}\right|^{-1}}
$$

Two different tolerance types are allowed.

- [type]

Type: attribute string

Default: "loc"

Description:

- If "loc": the time step is calculated in each subdomain and the smallest one is selected to maintain the error below the tolerance for all of them.

- If "tot": the total fission power is used to calculate the new the time step.

- <pow1_toll>

Type: real

Default: -1

Description: if $>0$ the time step will be calculated to maintain the truncation error on the flux smaller than the tolerance " $\tau$ ".

$$
\Delta t^{(n+2)}=2 \tau\left|\frac{\partial^{2} P^{(n+1)}}{\partial t^{2}}\right|^{-1}
$$

The tolerance is normalized with the total power of the first iteration, therefore the tolerance must be specified as a relative value (e.g. $1 \mathrm{e}-1,1 \mathrm{e}-3,1 \mathrm{e}-4 \ldots$ ) of the initial total power. Two different tolerance types are allowed.

- [type]

Type: attribute string

Default: "loc"

Description:

- If "loc": the time step is calculated in each subdomain and the smallest one is selected to maintain the error below the tolerance for all of them.

- If "tot": the total fission power is used to calculate the new the time step.

- <Methodology_02>

Description: The second methodology is based on the truncation error estimation using the second derivative of the flux/power. To estimate the second derivative a combination of backward and forward first order schemes is used:

$$
\left.\frac{\partial^{2} \phi}{\partial t^{2}}\right|_{t=t_{n+1}}=2 \frac{\Delta t_{n} \phi^{(n+1)}-\left(\Delta t_{n}+\Delta t_{n+1}\right) \phi^{(n)}+\Delta t_{n+1} \phi^{(n-1)}}{\Delta t_{n+1}{ }^{2} \Delta t_{n}}
$$

The block element and description is identical to the $<$ Methodology_01 $>$ block.

INL_Contract_Technical_Report_MS1_v2.3_GS_RB.docx

\section{Appendix B}


- <Methodology_03>

Description: The third methodology is based on the truncation error estimation using the second derivative of the flux/power. To estimate the second derivative a combination of backward and centered first order schemes is used:

$$
\left.\frac{\partial^{2} \phi}{\partial t^{2}}\right|_{t=t_{n+1}}=2 \frac{\Delta t_{n} \phi^{(n+1)}-\left(\Delta t_{n}+\Delta t_{n+1}\right) \phi^{(n)}+\Delta t_{n+1} \phi^{(n-1)}}{\Delta t_{n}\left(2 \Delta t_{n+1}{ }^{2}-\Delta t_{n}{ }^{2}\right)}
$$

The block element and description is identical to the <Methodology_01 $>$ block.

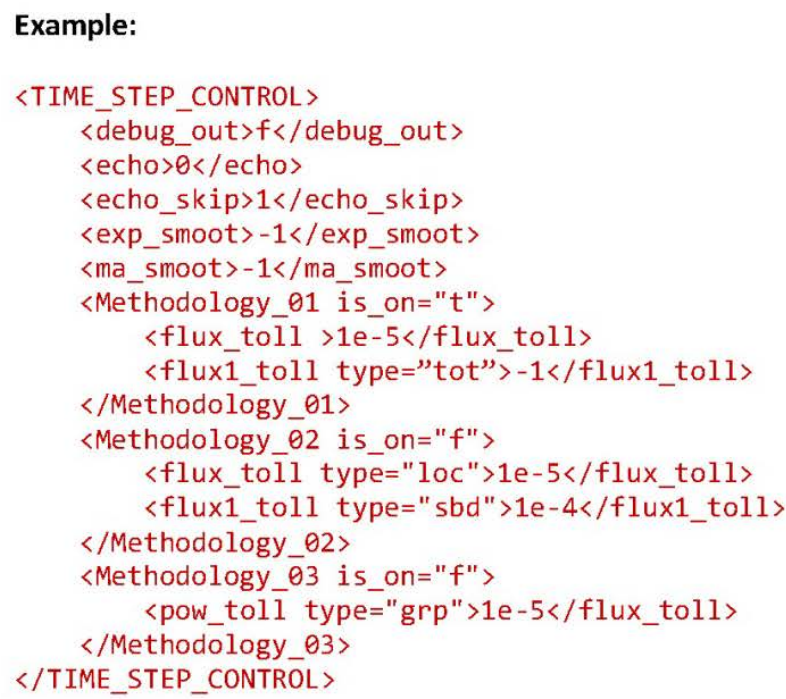

The sample input uses Methodology 1 with a local relative tolerance of $1 e-5$. These settings returned the best test results for the simplified HTTR model (see section 8), and is therefore recommended for use. A sensitivity study is suggested on the tolerance value to avoid that the calculated time step exceeds the thresholds defined in cards $\underline{2201-2299}$.

\subsubsection{Time step control “_dtk.csv" file description}

The file structure is described in table 7.3 below:

\begin{tabular}{|l|l|}
\hline First line & Alphanumeric part of the variables name (NAME) \\
\hline Second line & Numeric part of the variables name (CODE) \\
\hline Next lines & The data for each TH/NK iteration \\
\hline
\end{tabular}

Table 7.3 - “_dtk.csv" file structure

Table 7.4 explains the meaning of the variables printed in the "_dtk.csv". The alphanumeric part of the variable name is composed of 9 characters (one for the comma except the first variable in which there is a space instead, and 8 for the rest of the word).

INL_Contract_Technical_Report_MS1_v2.3_GS_RB.docx

Page 20 of 36

\section{Appendix B}




\begin{tabular}{|c|c|c|}
\hline NAME & CODE & DESCRIPTION \\
\hline \multicolumn{3}{|r|}{ GENERAL VARIABLES } \\
\hline timehy & 0 & TH problem time. (s) \\
\hline$d t$ & 0 & TH problem time step. (s) \\
\hline timekn & 0 & NK problem time. (s) \\
\hline dtkn & 0 & NK time step. (s) \\
\hline timekx & 0 & $\begin{array}{l}\text { For now, it is identical to "timekn". When the flux extrapolation is } \\
\text { implemented it will represent the NK time or the TH time, depending } \\
\text { on whether an advancement step or an extrapolation step is being } \\
\text { performed. (s) }\end{array}$ \\
\hline Ikinadv & 0 & $\begin{array}{l}\text { Logical variable for tracking the kinetics advancement. A value of } 0 \\
\text { implies that the kinetics solution is not advanced. }\end{array}$ \\
\hline Ikinsub & 0 & $\begin{array}{l}\text { Logical variable for determining if the NK is sub-cycling the TH. A value } \\
\text { of } 0 \text { implies that the NK time step is bigger than the TH one, a value of } \\
1 \text { implies that the NK time step is smaller than the TH one. }\end{array}$ \\
\hline Isync & 0 & $\begin{array}{l}\text { Logical variable for determining if the } \mathrm{TH} \text { and } \mathrm{NK} \text { calculations are } \\
\text { synchronized. A value of } 0 \text { implies that the two physics are not } \\
\text { synchronized; a value of } 1 \text { implies that the two physics are synchronized. }\end{array}$ \\
\hline fluxave & 0 & $\begin{array}{l}\text { Full domain average flux: } \\
\qquad \bar{\phi}^{(n)}=\frac{\sum_{r=1}^{N_{s b d}} \sum_{g=1}^{N_{g}}{ }_{r}^{g} \phi^{(n)} V_{r}}{\sum_{r=1}^{N_{s b d}} V_{r}} \\
\text { Where: " } N_{g} \text { " is the number of groups, "N } N_{s b d} \text { " is the number of } \\
\text { subdomains, " } V_{r} \text { " is the subdomain volume, " }{ }_{r}^{g} \phi(n) " \text { is the flux per } \\
\text { group and subdomain at the time step " } \mathrm{n} \text { ", " } \bar{\phi}(n) \text { " is the average flux. } \\
\text { (N/cm2 s) }\end{array}$ \\
\hline fipow & 0 & Total fission power. (W) \\
\hline \multicolumn{3}{|r|}{ METHODOLOGY SPECIFIC VARIABLES } \\
\hline 2 drvtv & Imno & $\begin{array}{l}\text { Second derivative of the flux or the power depending on the tolerance } \\
\text { type (flux_toll, flux1_toll or pow_toll, pow } 1 \text { _toll). } \\
\text { The numeric part of the name "Imno" is composed by four integers } \\
\text { described below: } \\
\text { - I: is the Methodology number }(1 \leq \mid \leq 3) \text {, } \\
\text { - } \mathbf{m}: 1 \text { is for a flux tolerance (flux_toll, flux } 1 \text { toll) and } 2 \text { is for a } \\
\text { power tolerance (pow_toll, pow } 1 \text { toll) }(1 \leq \mathrm{m} \leq 2) \text {, } \\
\text { - } \mathbf{n}: 1 \text { is for the integrated truncation error tolerance (flux_toll, } \\
\text { pow_toll), } 2 \text { is for an absolute truncation error tolerance } \\
\text { (flux1_toll, pow } 1 \text { toll) }(1 \leq n \leq 2) \text {, }\end{array}$ \\
\hline
\end{tabular}

INL_Contract_Technical_Report_MS1_v2.3_GS_RB.docx 


\begin{tabular}{|c|c|c|}
\hline & & $\begin{array}{l}\text { - o: } 1 \text { is for "loc" type of tolerance, } 2 \text { is for "grp" type of } \\
\text { tolerance, } 3 \text { is for "sbd" type of tolerance, } 4 \text { is for "tot" type of } \\
\text { tolerance }(1 \leq 0<\leq) \text {. (see } 7.3 .2)\end{array}$ \\
\hline dtknp & Imno & $\begin{array}{l}\text { Predicted time step. This time is different from "timekn" because } \\
\text { instead of using the value of dtknp directly, it is smoothed to be a } \\
\text { rational multiple or divisor of the TH time step. The numeric part of the } \\
\text { name "Imno" is the same of the 2drvtv variable. }\end{array}$ \\
\hline min_sd & Imno & $\begin{array}{l}\text { This variable is present only for the "loc" and "grp" type of tolerances } \\
\text { and describe the selected subdomain number for the time step } \\
\text { calculation. The numeric part of the name "Imno" is the same of the } \\
\text { "2drvtv" variable. }\end{array}$ \\
\hline $\min \_g$ & Imno & $\begin{array}{l}\text { This variable is present only for the "loc" and "sbd" type of tolerances } \\
\text { and describe the selected group number for the time step calculation. } \\
\text { The numeric part of the name "Imno" is the same of the "2drvtv" } \\
\text { variable. }\end{array}$ \\
\hline
\end{tabular}

Table 7.4 - “_dtk.csv” variables description

\section{Code validation}

\subsubsection{The test Case}

The High Temperature Engineering Test Reactor (HTTR) is a High Temperature Gas Cooled Reactor (HTGR) built in Japan at the Oarai Research and Development Center of Japan Atomic Energy Agency (JAEA). It reached full design power of $30 \mathrm{MW}$ in 1999. The main design parameters of the reactor and a component view are shown in Table 8.1 and Figure 8.1.
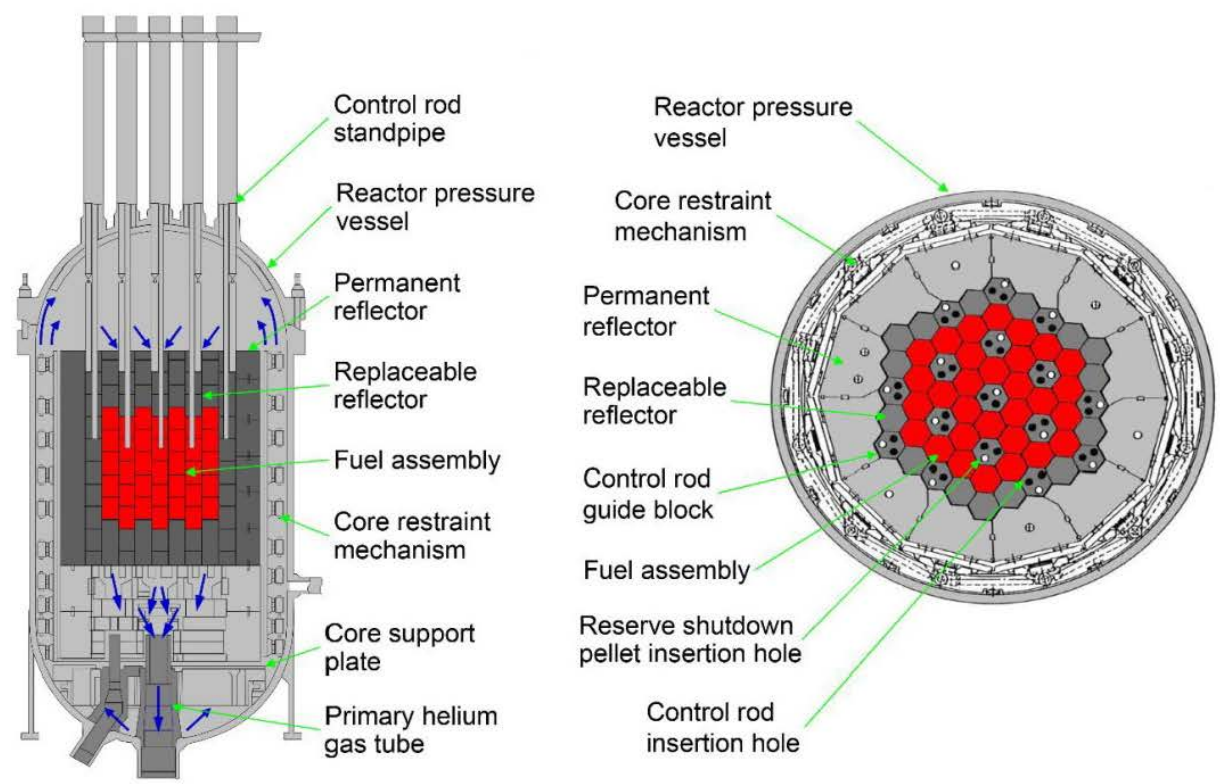

INL_Contract_Technical_Report_MS1_V2.3_GS_RB.docx

Page 22 of 36 
Figure 8.1 - Structure of the HTTR reactor

\begin{tabular}{|l|l|}
\hline \multicolumn{2}{|c|}{ MAIN REACTOR PARAMETERS } \\
\hline Rated Power & $30 \mathrm{MW}$ \\
\hline Coolant & Helium \\
\hline Moderator & Graphite \\
\hline Fuel & $\begin{array}{l}\text { UO }{ }_{2} \text { ceramic-coated particle fuel pin in } \\
\text { block }\end{array}$ \\
\hline Primary pressure & $4.0 \mathrm{MPa}$ \\
\hline Average power density & $2.5 \mathrm{~W} / \mathrm{cm}^{3}$ \\
\hline Core diameter & $2.9 \mathrm{~m}$ \\
\hline Outlet coolant temperature & $850^{\circ} \mathrm{C}$ \\
\hline Inlet coolant temperature & $395^{\circ} \mathrm{C}$ \\
\hline
\end{tabular}

Table 8.1- HTTR full power parameters

In 2010, a Loss of Forced Cooling (LOFC) test was performed at the HTTR to demonstrate advanced reactor safety characteristics. LOFC \#1 was performed at an initial power of $9 \mathrm{MW}$ (see Figure 8.2) and has been selected as a test case to benchmark the $P / R$ best estimate code capabilities.

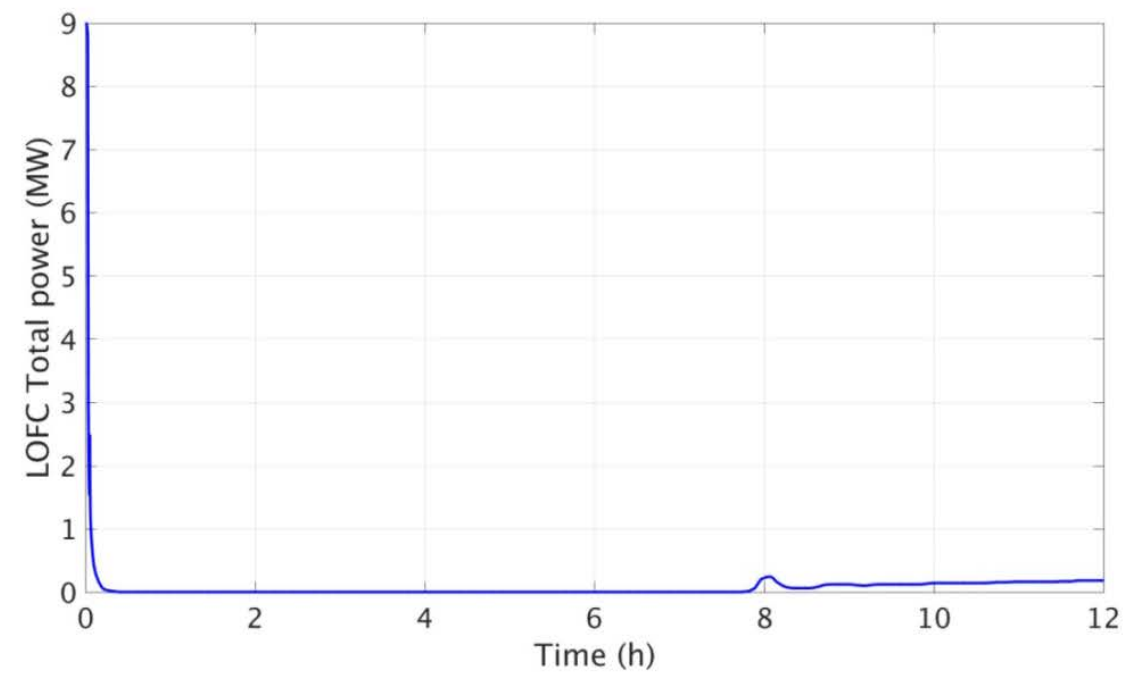

Figure 8.2 - Loss of forced cooling test at $9 \mathrm{MW}$ Total Power

After the coolant mass flow rate reaches zero, the reactor power decrease due to the negative reactivity insertion from Doppler feedback as fuel temperature increased. After that the

INL_Contract_Technical_Report_MS1_V2.3_GS_RB.docx

Page 23 of 36

Appendix B 
reactor starts to cools down but the xenon buildup maintain the reactor sub critical. The xenon concentration starts to decrease after about 7 hours and the reactor becomes critical again after 8 hours, as shown in Figure 8.2. A 3D NK-TH model has been developed with the $\mathrm{P} / \mathrm{R}$ coupled code to simulate the transient. The DRAGON5 cross-section library has been tabulated as functions of 4 fuel and 4 moderator temperatures and 3 Xenon concentrations to track the xenon and temperature reactivity changes that determine the re-criticality time. To test the adaptive time step feature, a simplified version of this model has been developed and used to simulate the first 1,000 s of the transient. The simplified model consists of only one assembly of the HTTR but the transient boundary conditions and the cross-section library are the same.

\subsubsection{Analysis and results for all the methodologies}

All the available combinations of tolerance types and methodologies have been tested. For each calculation in the figures below a tolerance of $1 \mathrm{e}-4$, a minimum and maximum time step of $1 \mathrm{e}-3 \mathrm{~s}$ and $2 \mathrm{~s}$ and a TH time step of $1 \mathrm{e}-3 \mathrm{~s}$ have been imposed. A LOFC simulation with a constant NK and TH time step of $1 \mathrm{e}-3$ s has been used as the reference solution. As visible in the figures for all the methodologies except for the absolute tolerance imposed on the local power "abs-pow-loc", the plotted lines overlap perfectly with the reference solution, indicating a very small absolute error. Analyzing the relative error instead for all the cases in which an absolute tolerance "abs-" has been used provides interesting trends. For these cases the relative error remains under a few percentage points only at the beginning of the transient. As the flux decreases, the time step increases until the limit of $2 \mathrm{~s}$ and the relative error increases rapidly. It should, however, be noted that even if the last part of the transient is affected by a large relative error the absolute error is still very small.

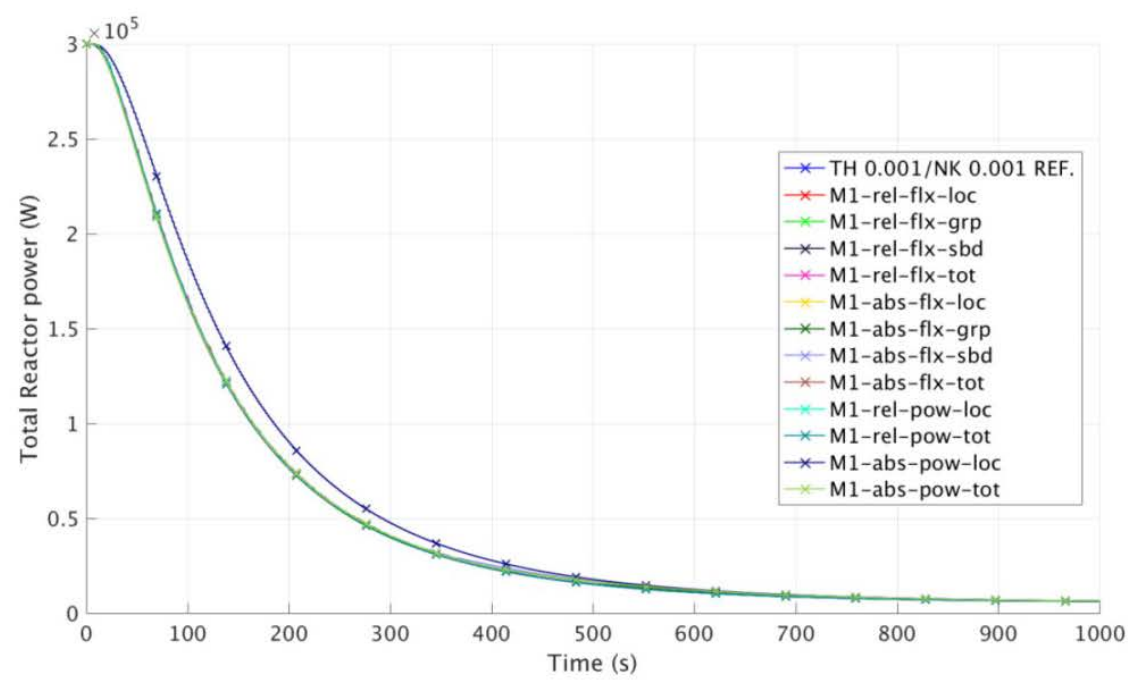

Figure 8.3 - Methodology 01 total power $(\tau=1 \mathrm{e}-4)$

INL_Contract_Technical_Report_MS1_v2.3_GS_RB.docx 


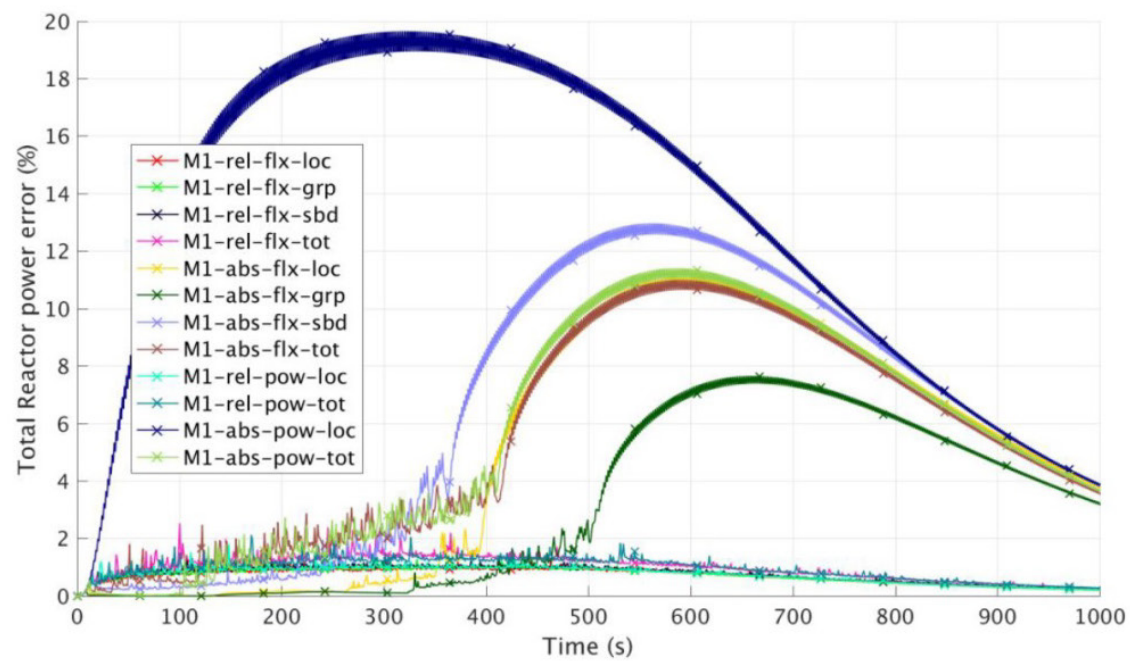

Figure 8.4 - Methodology 01 total power relative error $(\tau=1 \mathrm{e}-4)$

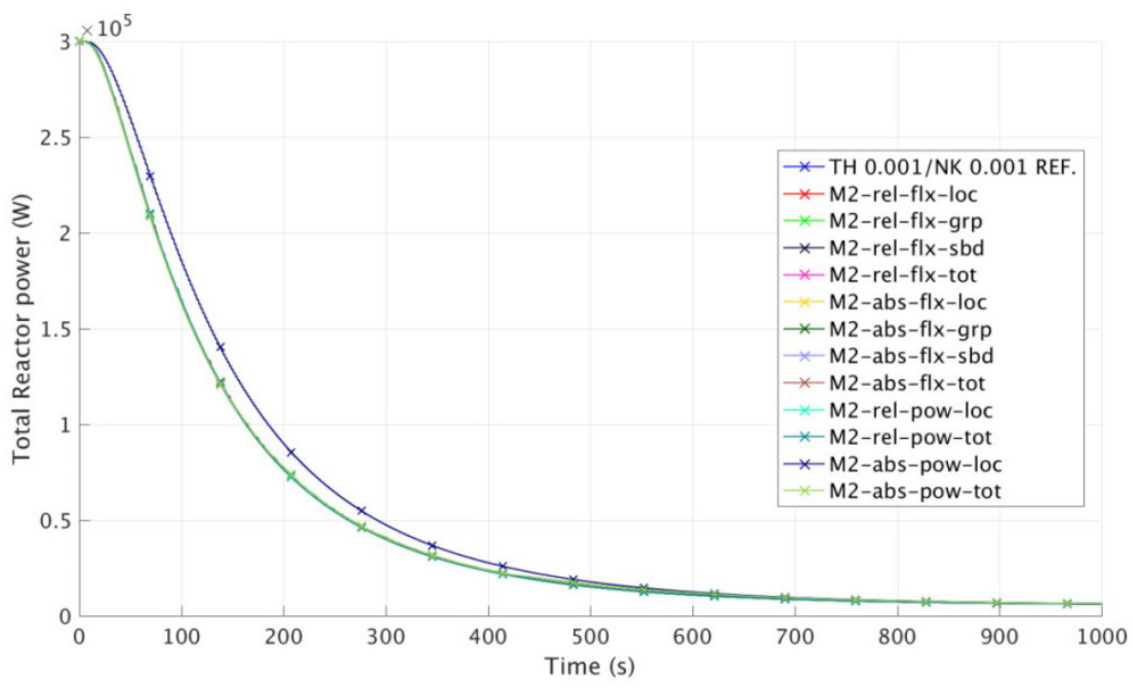

Figure 8.5 - Methodology 02 total power $(\tau=1 \mathrm{e}-4)$

L_Contract_Technical_Report_MS1_v2.3_GS_RB.docx 


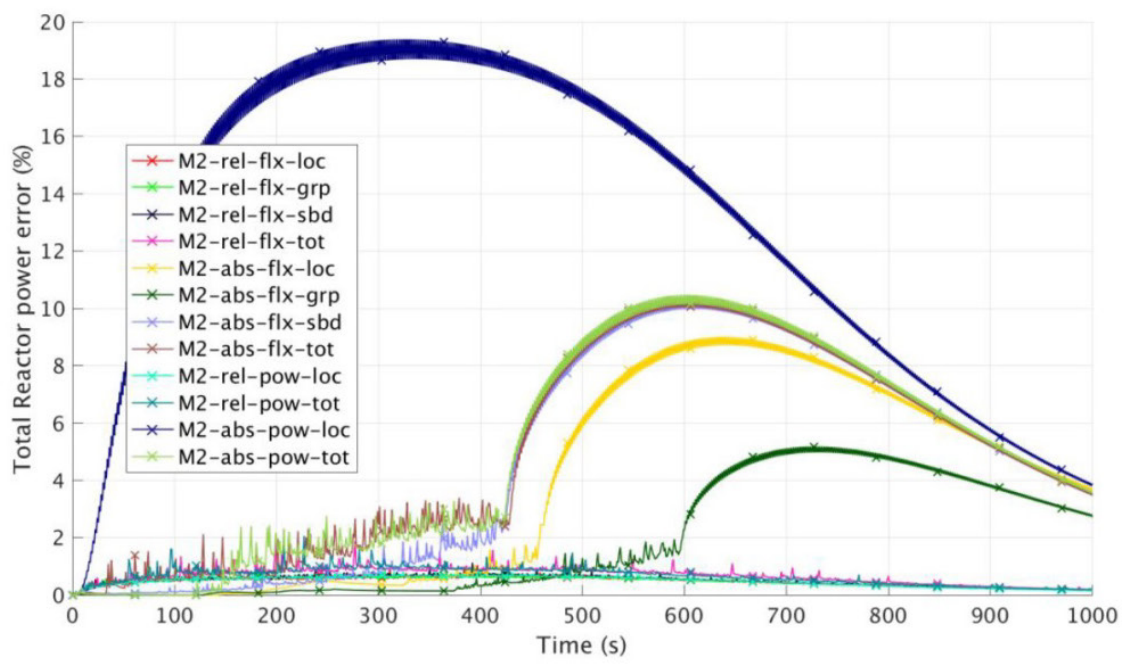

Figure 8.6 - Methodology 02 total power relative error $(\tau=1 \mathrm{e}-4)$

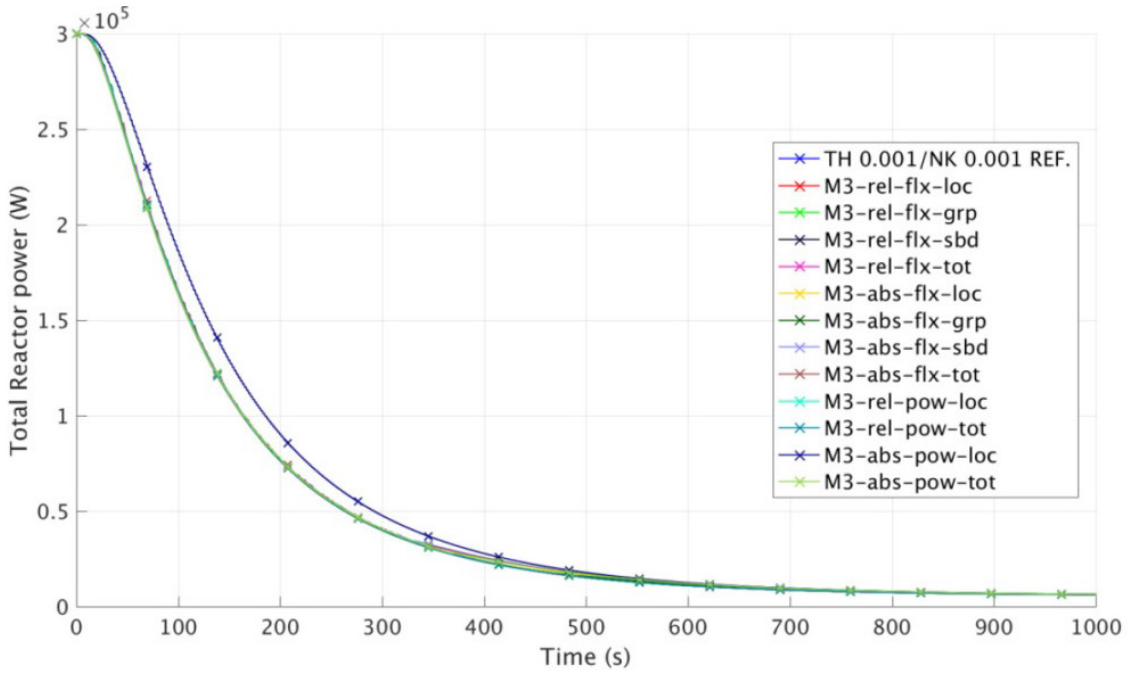

Figure 8.7 - Methodology 03 total power $(\tau=1 \mathrm{e}-4)$

\section{-_Contract_Technical_Report_MS1_v2.3_GS_RB.docx}




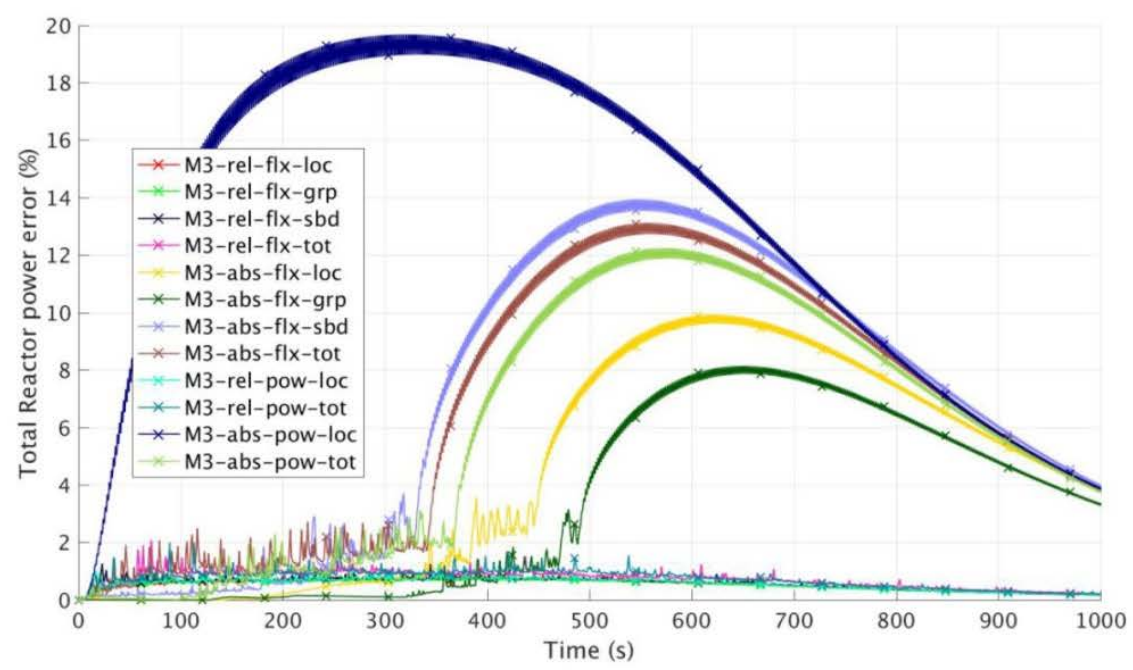

Figure 8.8 - Methodology 03 total power relative error $(\tau=1 \mathrm{e}-4)$

\begin{tabular}{|l|c|l|c|l|c|}
\hline \multicolumn{1}{|c|}{ NAME } & SPEEDUP & \multicolumn{1}{c|}{ NAME } & SPEEDUP & \multicolumn{1}{|c|}{ NAME } & SPEEDUP \\
\hline M1-rel-flx-loc & 17 & M2-rel-flx-loc & 12 & M3-rel-flx-loc & 13 \\
\hline M1-rel-flx-grp & 17 & M2-rel-flx-grp & 12 & M3-rel-flx-grp & 13 \\
\hline M1-rel-flx-sbd & 18 & M2-rel-flx-sbd & 12 & M3-rel-flx-sbd & 14 \\
\hline M1-rel-flx-tot & 18 & M2-rel-flx-tot & 12 & M3-rel-flx-tot & 15 \\
\hline M1-abs-flx-loc & 6 & M2-abs-flx-loc & 4 & M3-abs-flx-loc & 6 \\
\hline M1-abs-flx-grp & 5 & M2-abs-flx-grp & 3 & M3-abs-flx-grp & 4 \\
\hline M1-abs-flx-sbd & 44 & M2-abs-flx-sbd & 23 & M3-abs-flx-sbd & 44 \\
\hline M1-abs-flx-tot & 31 & M2-abs-flx-tot & 20 & M3-abs-flx-tot & 43 \\
\hline M1-rel-pow-loc & 17 & M2-rel-pow-loc & 12 & M3-rel-pow-loc & 14 \\
\hline M1-rel-pow-tot & 18 & M2-rel-pow-tot & 13 & M3-rel-pow-tot & 15 \\
\hline M1-abs-pow-loc & 165 & M2-abs-pow-loc & 165 & M3-abs-pow-loc & 165 \\
\hline M1-abs-pow-tot & 18 & M2-abs-pow-tot & 12 & M3-abs-pow-tot & 19 \\
\hline
\end{tabular}

Table 8.2 - Methodologies 1,2 and 3 speedups.

It is shown in Table 8.2 that the use of a relative tolerance "rel-" of 1e-4 allows the relative error to remain under $1 \%$ while obtaining a very good speedup between 12 and 18 . Using an absolute tolerance of 1e-4 the speed up in some cases is smaller (as for "abs-flx-loc") and in other cases bigger ( "M1-abs-flx-tot").

The speedup of 165 for the "abs-pow-loc" case is too big and results from a tolerance that was too large resulting in imposing the maximum time step from the beginning. It has to be noted though that even for this case the absolute error remains relatively small (under 10\%). This "abs-pow-loc" option can be therefore possibly be utilized for early scoping calculations

INL_Contract_Technical_Report_MS1_V2.3_GS_RB.docx

Page 27 of 36

\section{Appendix B}


where simulation time and model development turn-around is more important than very accurate results.

\subsubsection{Methodology stability analysis}

\section{Local Relative Tolerance}

It is shown in Figure 8.10 and Table 8.3 that using a tolerance of $1 \mathrm{e}-5$ it is possible to maintain the relative error under the $0.35 \%$ and obtain a speedup from 5 to 7 . The time step prediction as explained in paragraph 6.3 is affected by some instabilities. Figure 8.11 shows that the predicted time step for all three methodologies has a noise that is mostly produced by the noise on the flux second derivative estimation as shown in Figure 8.12. The second methodology seems to be the most stable option - it results in a time step with less noise than the other methods but also with a quite smaller speedup.

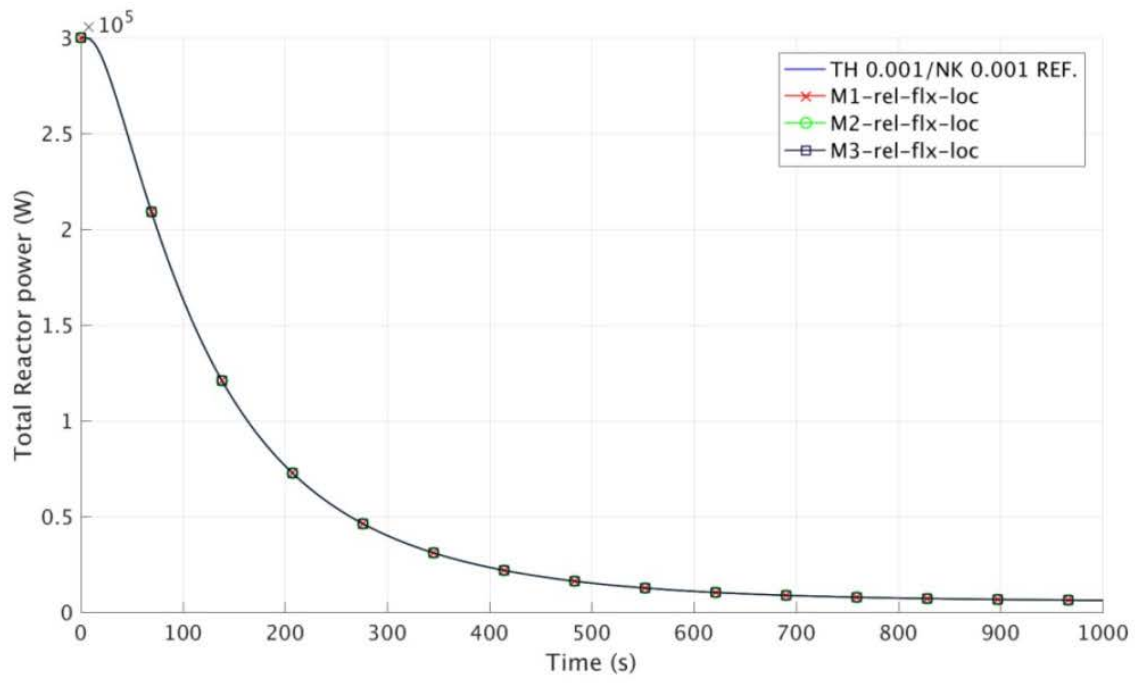

Figure 8.9- Methodologies 01,02 and 03 total power with a relative tolerance of $\tau=1 \mathrm{e}-5$

INL_Contract_Technical_Report_MS1_V2.3_GS_RB.docx 


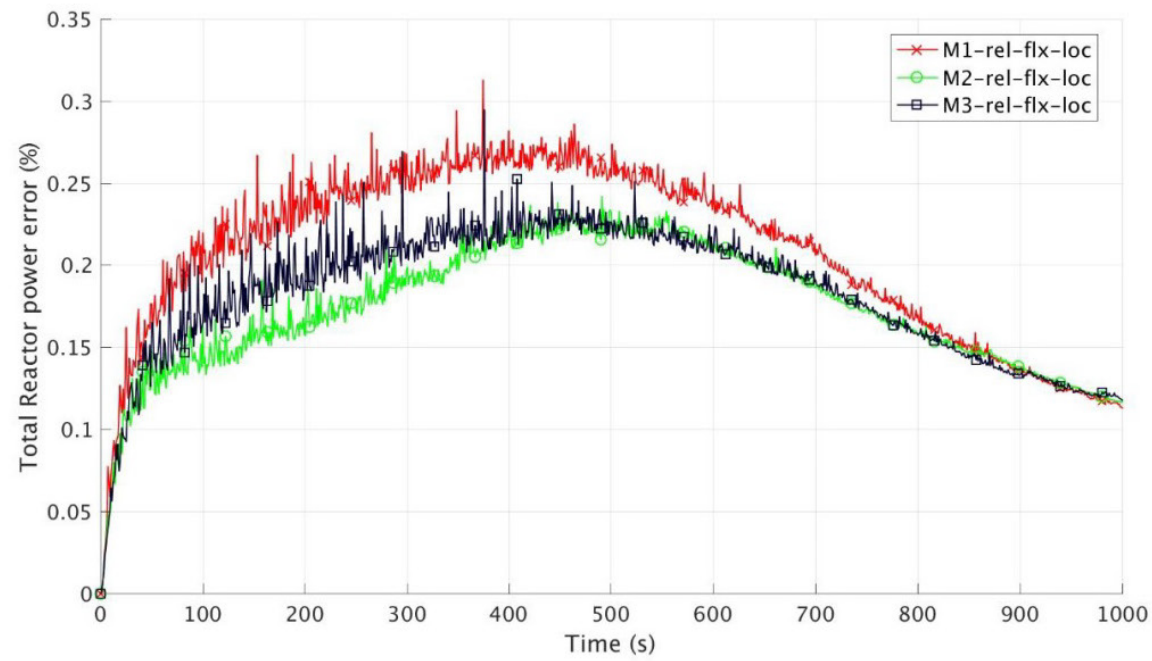

Figure 8.10 - Methodologies 01,02 and 03 total power relative error with a relative tolerance of $\tau=1 e-5$

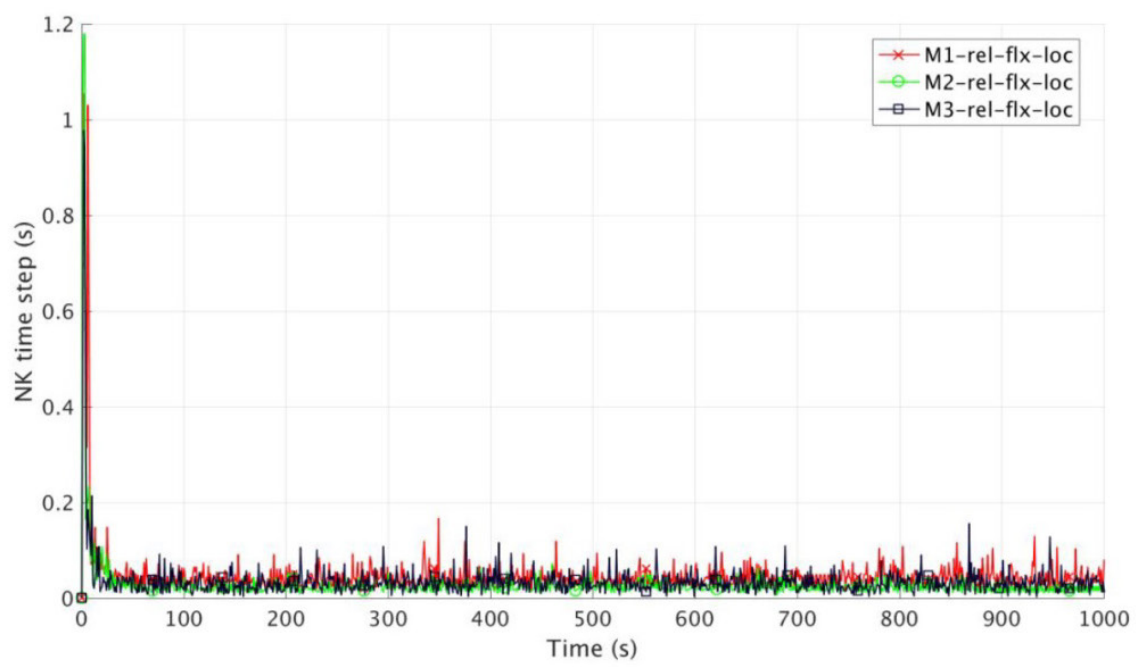

Figure 8.11 - Methodologies 01,02 and 03 neutronics time step, relative tolerance $(\tau=1 \mathrm{e}-5)$

NL_Contract_Technical_Report_MS1_v2.3_GS_RB.docx

Appendix B 


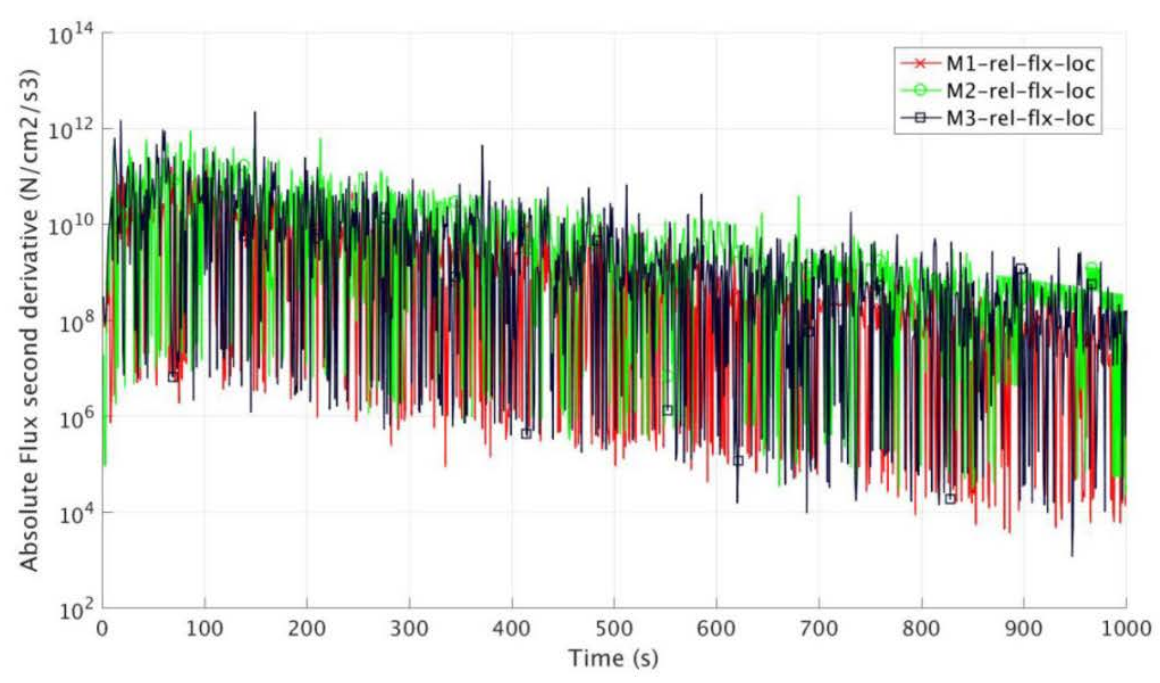

Figure 8.12 - Methodology $01,02,03$ flux second time derivative, relative tolerance $(\tau=1 \mathrm{e}-5)$

\begin{tabular}{|c|c|c|c|c|c|}
\hline NAME & SPEEDUP & NAME & SPEEDUP & NAME & SPEEDUP \\
\hline M1-rel-flx-loc & 7 & M2-rel-flx-loc & 5 & M3-rel-flx-loc & 6 \\
\hline
\end{tabular}

Table 8.3 - Methodologies 01, 02 and 03 local relative tolerance on the flux speedups $(\tau=1 \mathrm{e}-5)$.

8.1.3.2

\section{Local Absolute Tolerance}

Figure 8.14 and Table 8.4 show that it is possible to maintain the relative error under $2 \%$ for the first $400 \mathrm{~s}$ using a tolerance of $1 \mathrm{e}-4$, after which the power becomes one tenth of the starting value and the time step increases until the limit of $2 \mathrm{~s}$. The speedup is smaller (between 4 and 6), but even in this case the predicted time step is noisy and depends on the second derivative estimation noise. Figure 8.16 shows that the noise associated with the flux second derivative estimation disappears when the constant maximum time step is used. This happens because the estimation of the time second time derivative using equations (18), (27) and (28) (that converge to the same value) is more accurate.

INL_Contract_Technical_Report_MS1_v2.3_GS_RB.docx 


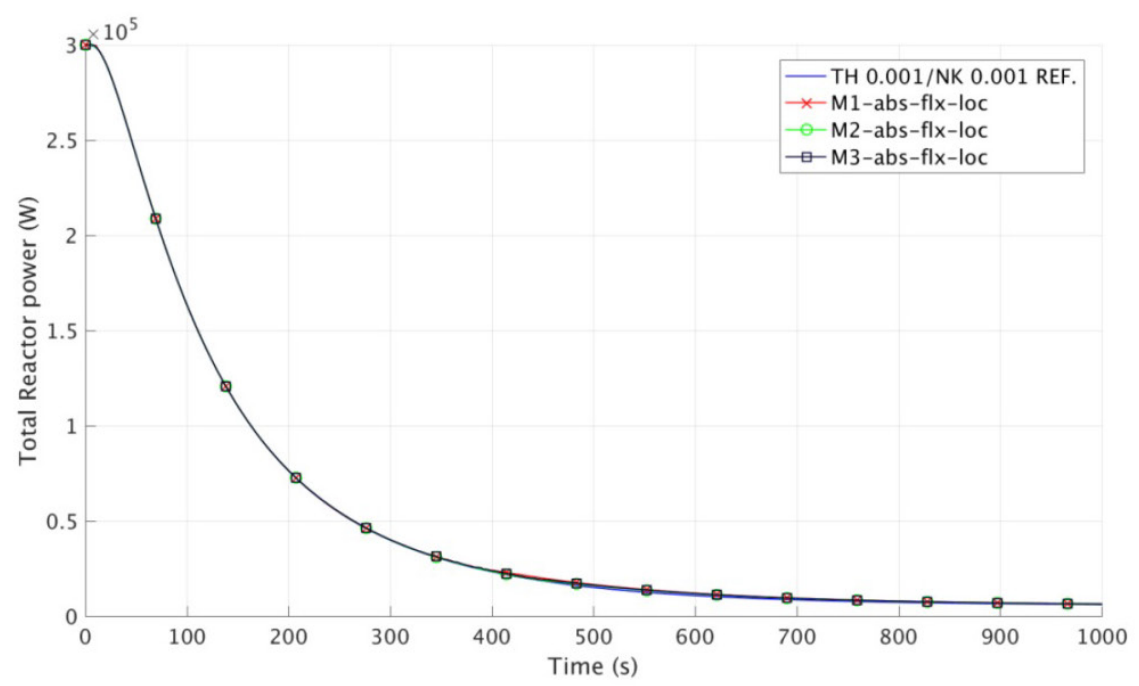

Figure 8.13 - Methodologies 01,02 and 03 total power, absolute tolerance $(\tau=1 e-4)$

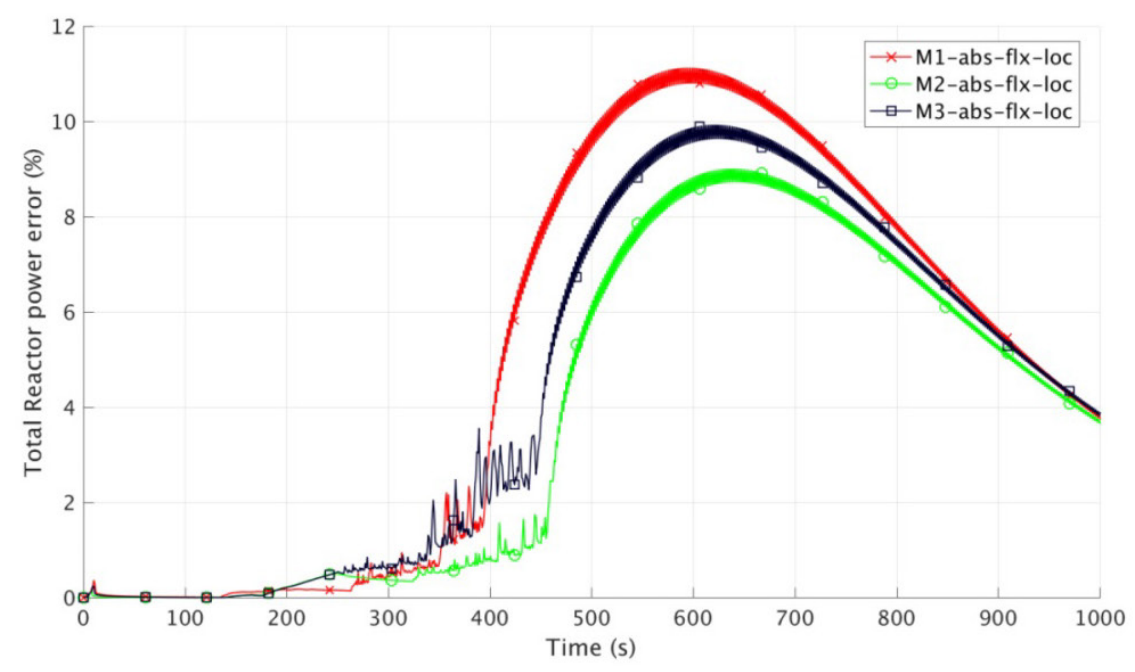

Figure 8.14 - Methodologies 01,02 and 03 total power relative error, absolute tolerance $(\tau=1 \mathrm{e}-4)$

\section{INL_Contract_Technical_Report_MS1_v2.3_GS_RB.docx}




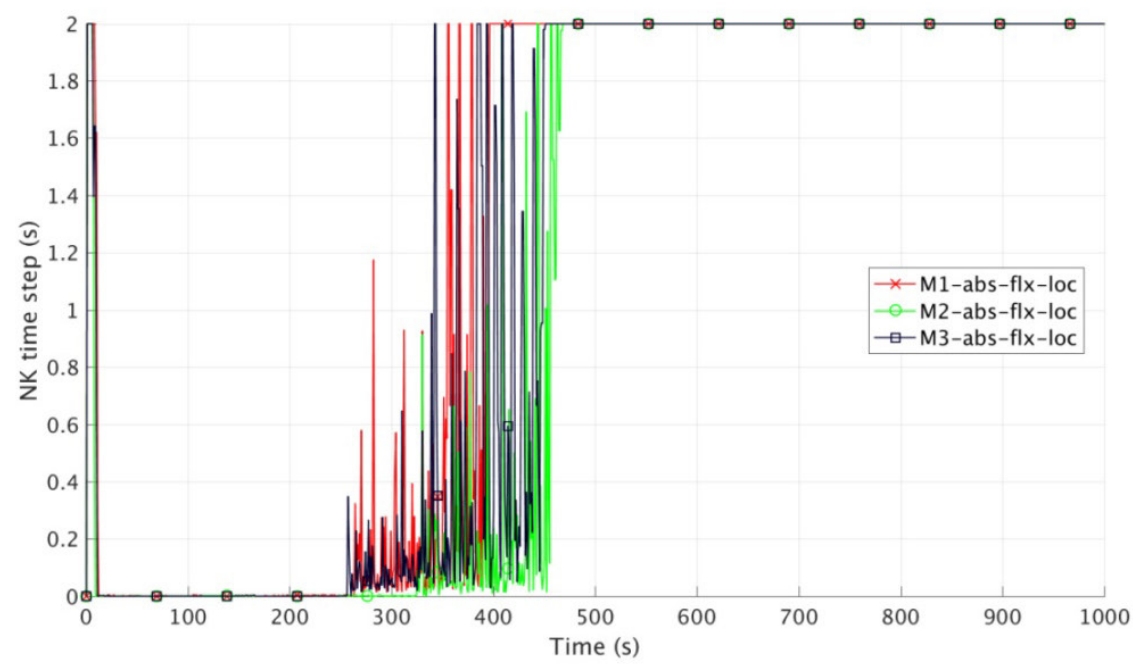

Figure 8.15 - Methodologies 01,02 and 03 neutronics time step, absolute tolerance $(\tau=1 \mathrm{e}-4)$

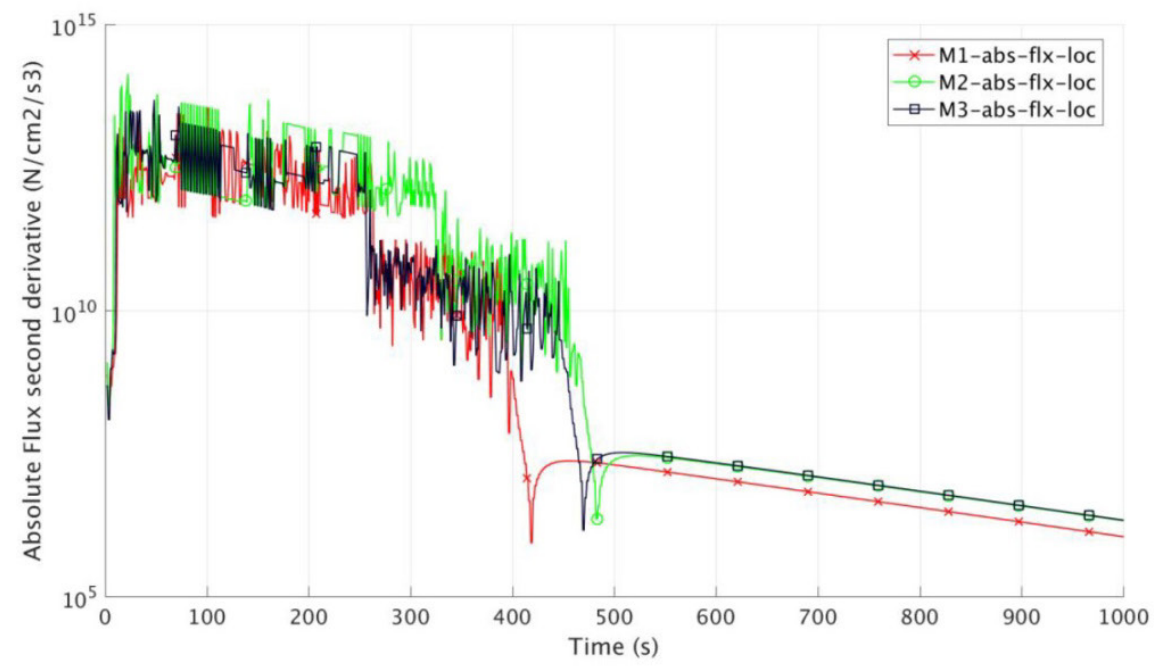

Figure 8.16 - Methodologies 01,02 and 03 flux second time derivative, absolute tolerance $(\tau=1 \mathrm{e}-4)$

\begin{tabular}{|c|c|c|c|c|c|}
\hline NAME & SPEEDUP & NAME & SPEEDUP & NAME & SPEEDUP \\
\hline M1-rel-flx-loc & 6 & M2-rel-flx-loc & 4 & M3-rel-flx-loc & 6 \\
\hline
\end{tabular}

Table 8.4 - Methodologies 01, 02 and 03 local absolute tolerance on the flux speedups $(\tau=1 \mathrm{e}-4)$.

\section{INL_Contract_Technical_Report_MS1_V2.3_GS_RB.docx}




\subsubsection{The time step smoothing}

To increase the stability of the time step calculation two smoothing approaches has been introduced. The first one is an exponential smoothing with coefficient " $\alpha$ " as described in (34) and the second a moving average of degree " $N_{M A}$ " as described in (35).

$$
\begin{aligned}
\Delta t_{n+2} & =\Delta t_{n+1}(1-\alpha)+\alpha \Delta t_{p} \\
\Delta t_{n+2} & =\frac{\sum_{i=1}^{N_{M A}-1} \Delta t_{n+2-i}+\Delta t_{p}}{N_{M A}}
\end{aligned}
$$

Where " $\Delta t{ }_{p}$ " it the predicted time step without smoothing. Using a moving average of order 8 the speedup increase to 11 (see Table 8.5) and the time step is more stable as expected (see Figure 8.19) but the relative error (see Figure 8.18) is larger especially in the first part of the transient. Using the exponential smoothing with a coefficient of 0.5 the speedup increase to 8 (see Table 8.5) and the relative error is comparable with the calculation without smoothing (see Figure 8.18), but the time step is quite more stable. In each case the second derivative estimation is not affected (see Figure 8.20).

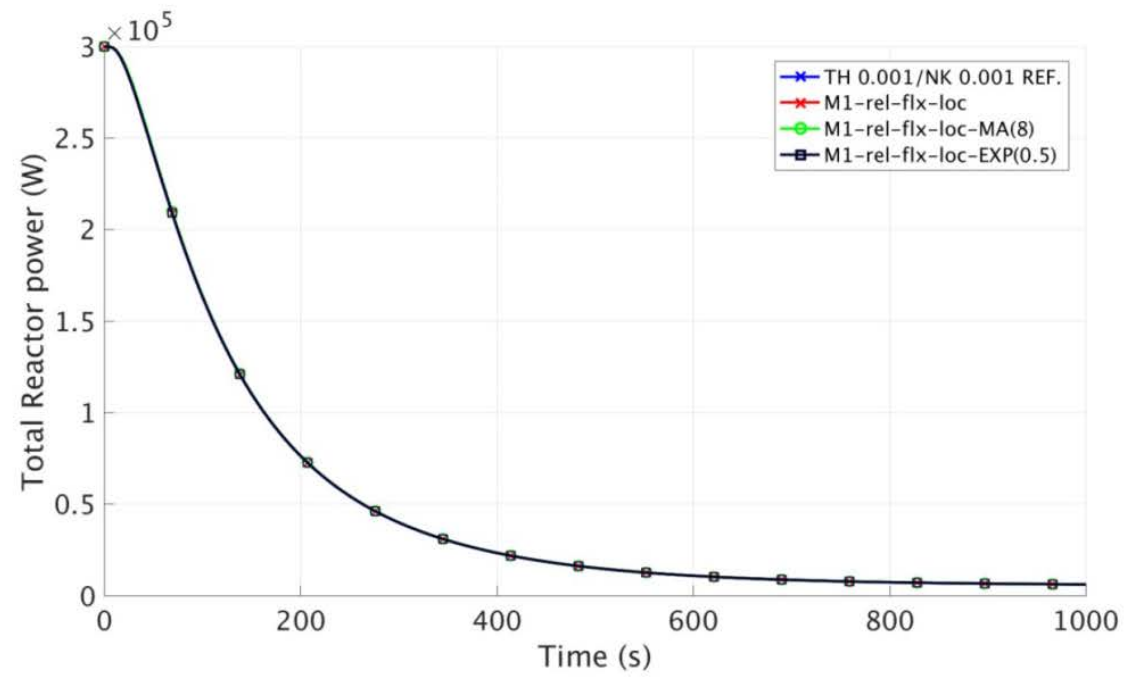

Figure 8.17 - Methodology 01 plus moving average and exponential smoothing total power $(\tau=1 \mathrm{e}-5)$ 


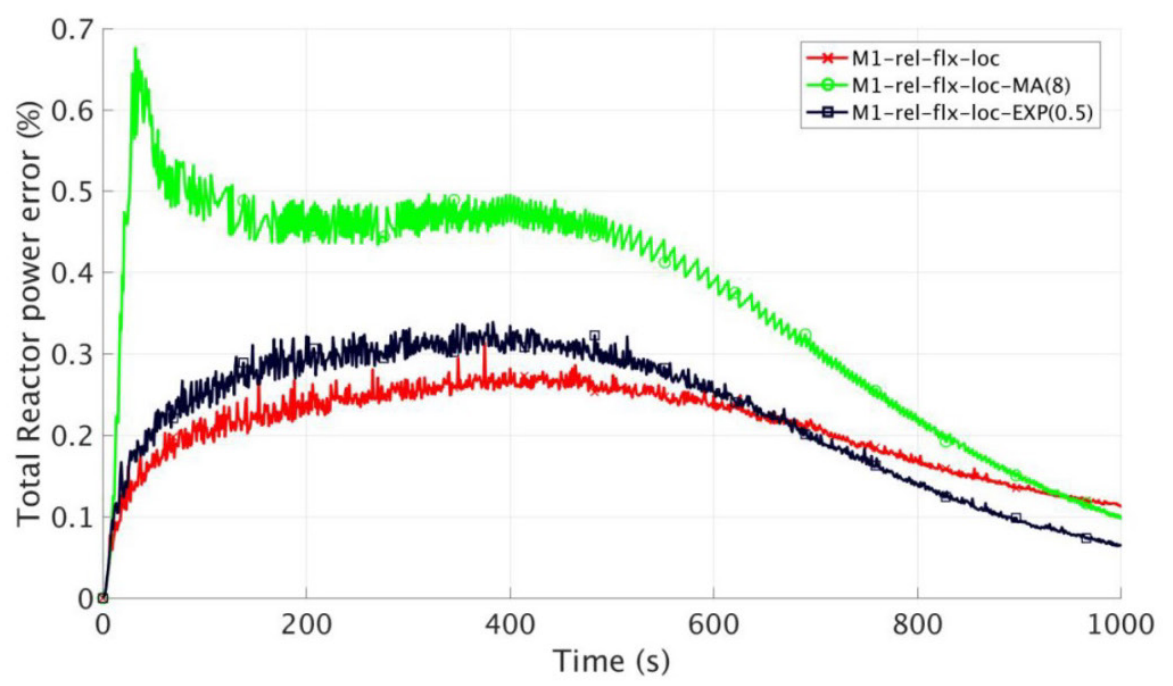

Figure 8.18 - Methodology 01 plus moving average and exponential smoothing total power relative error $(\tau=1 \mathrm{e}-5)$

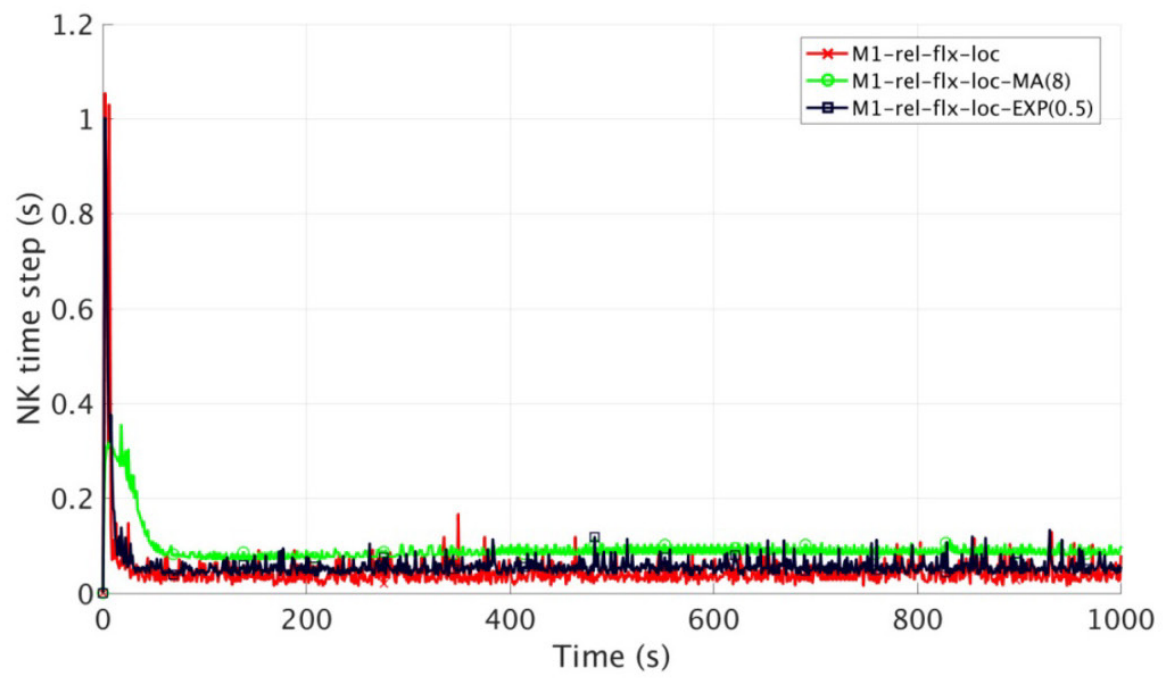

Figure 8.19 - Methodology 01 moving average and exponential smoothing neutronics time step ( $\tau=1 \mathrm{e}-5)$

INL_Contract_Technical_Report_MS1_v2.3_GS_RB.docx 


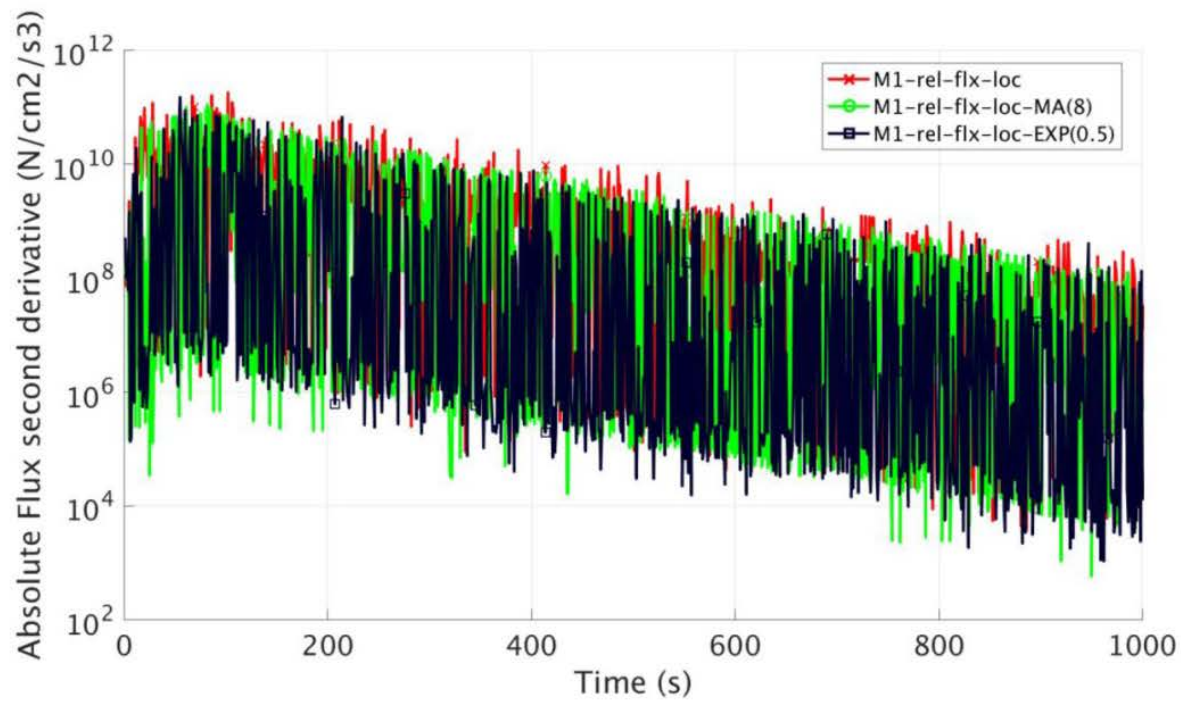

Figure 8.20 - Methodology 01 moving average and exponential smoothing flux second time derivative $(\tau=1 \mathrm{e}-5)$

\begin{tabular}{|c|c|l|c|l|c|}
\hline NAME & SPEEDUP & \multicolumn{1}{|c|}{ NAME } & SPEEDUP & \multicolumn{1}{c|}{ NAME } & SPEEDUP \\
\hline M1-rel-flx-loc & 7 & $\begin{array}{l}\text { M1-rel-flx-loc- } \\
\text { MA(8) }\end{array}$ & 11 & $\begin{array}{l}\text { M1-rel-flx-loc- } \\
\text { EXP(0.5) }\end{array}$ & 8 \\
\hline
\end{tabular}

Table 8.5 - Methodology 1 local relative tolerance on the flux and smoothing speedups $(\tau=1 \mathrm{e}-5)$.

\section{Conclusions}

The theory for the time step control has been analyzed and implemented in the P/R coupled code resulting in three different methodologies and 12 different ways to define a tolerance. More than 2,000 lines of code have been added. Some modifications to the original RELAP5$3 \mathrm{D}^{\odot}$ code and to the interface has been implemented. This document describes the theory and implementation of the adaptive time step module and how to use. The methodology has been successfully tested on a reduced LOFC P/R model of the Japanese HTTR, obtaining a good speedup while maintaining the relative error under $1 \%$. The best methodology for this test case seems to be the second methodology with a relative local tolerance on the flux of $1 \mathrm{e}-5$ that allows a speedup of 5 and a relative error of less than $0.3 \%$.

\section{References}

INL_Contract_Technical_Report_MS1_V2.3_GS_RB.docx 
[1] A. Epiney, C. Rabiti, A. Alfonsi, Y. Wang, J. Cogliati and G. Strydom, "PHISICS multi-group transport neutronic capabilities for RELAP5," in ICAPP 12, Chicago, USA, June 24-28, 2012.

[2] Y. Wang, C. Rabiti and G. Palmiotti, "Krylov Solvers Preconditioned with the Low-Order Red-Black Algorithm for The Pn Hybrid FEM for the INSTANT Code," in International Conference on Mathematics and Computational Methods Applied to Nuclear Science and Engineering, Rio de Janeiro, RJ, Brazil, May 8-12, 2011.

[3] C. Rabiti and A. Rineiski, "Extension of KIN3D, a Kinetics Capability of VARIANT, for Modeling Fast Transients in Accelerator Driven Systems," in PHYSOR 2004 - The Physics of Fuel Cycles and Advanced Nuclear Systems: Global Developments, Chicago, Illinois, April 25-29, 2004.

[4] C. Rabiti, Modelling of fast neutron transients in an accelerator driven system, Ph.D thesis, IKE, Institut für Kernenergetik und Energiesysteme der Universität Stuttgart, 10. November 2006.

[5] C. Rabiti, A. Alfonsi and A. Epiney, "New Simulation Schemes and Capabilities for the PHISICS/RELAP5-3D Coupled Suite," Nuclear Science and Engineering, Vols. Volume 182, Number 1, pp. Pages 104-118, January 2016.

[6] M. W. Hackemack and J. M. Pounders, "Implementation of an a priori time step estimator for the multigroup neutron diffusion equation in asynchronously coupled RELAP5-3D," in PHYSOR 2014 - The Role of Reactor Physics toward a Sustainable Future, The Westin Miyako, Kyoto, Japan , September 28 - October 3, 2014.

[7] INL, RELAP5-3D@ Code Manual Volume II: User's Guide and Input Requirements, Appendix A, RELAP5-3D/4.3, 2015.

[8] C. Rabiti, G. Lohnert, W. Maschek and A. Rineiski, "Time step control for solving the transient even-parity neutron transport equation," in Mathematics and Computation, Supercomputing, Reactor Physics and Nuclear and Biological Applications, Palais des Papes, Avignon, France, September 12-15, 2005.

INL_Contract_Technical_Report_MS1_v2.3_GS_RB.docx 